\author{
Universidade de São Paulo \\ Instituto de Física
}

\title{
Novos Modelos na Escala TeV para a Hierarquia de Gauge e das Massas dos Férmions.
}

\author{
Leonardo de Lima \\ Orientador: Prof. Dr. Gustavo Alberto Burdman \\ Tese de doutorado apresentada ao \\ Instituto de Física para a obtenção \\ do título de Doutor em Ciências.
}

\section{Banca Examinadora:}

Prof. Dr. Gustavo Alberto Burdman (IF/USP)

Prof. Dr. Oscar José Pinto Éboli (IF/USP)

Prof. Dr. Diego Trancanelli (IF/USP)

Prof. Dr. Rogério Rosenfeld (IFT/UNESP)

Prof. Dr. Alex Gomes Dias (UFABC)

São Paulo

2013 


\section{Resumo}

O Modelo Padrão (MP) da física de partículas fornece uma descrição extremamente bem sucedida de todos os dados experimentais atuais. No entanto, temos razões para esperar que ele não seja válido até escalas de energia arbitrariamente altas. No MP a massa do bóson de Higgs é quadraticamente sensível a correções radiativas, sofrendo de grandes problemas de ajuste fino. Isso gera o chamado problema da hierarquia de gauge, cuja solução natural requer nova física na escala TeV. O MP também não fornece nenhuma explicacão para a grande hierarquia de massas e ângulos de mistura dos férmions.

Os problemas de hierarquia podem ser resolvidos naturalmente em teorias com uma dimensão extra curva. No entanto, a sua versão mais simples viola sabor a nível árvore, em conflito com os dados. Nesta tese investigaremos modelos quadridimensionais que descrevem a física na escala TeV obtidos da discretização de teorias em cinco dimensões no espaço $A d S_{5}$, segundo o procedimento de desconstrução dimensional. As hierarquias de gauge e das massas dos férmions são geradas naturalmente como no modelo extra-dimensional, satisfazendo ainda os vínculos de sabor e de precisão eletrofraca. Estudaremos também um modelo desconstruído em que o Higgs é um pseudo bóson de Nambu-Goldstone, a fim de obter um Higgs naturalmente leve e dinamicamente localizado. 
Resumo 


\section{Abstract}

The Standard Model (SM) of particle physics provides an extremely successful description of all current experimental data. However, we have reasons to expect that it cannot be valid up to arbitrarily high energy scales. In the SM the Higgs boson mass is quadratically sensitive to radiative corrections and, hence, suffers from a severe fine-tunning problem. This creates the so-called gauge hierarchy problem, the natural solution of which requires new physics at the $\mathrm{TeV}$ scale. The SM also doesn't provide any explanation for the large hierarchy of fermion masses and mixing angles.

The hierarchy problems can be naturally solved in theories with a warped extra dimension. However, their simplest version violates flavor at tree level, conflicting with experimental data. In this thesis we investigate four dimensional models that describe physics at the $\mathrm{TeV}$ scale obtained from a coarse discretization of five dimensional theories in $A d S_{5}$ space, by the dimensional deconstruction procedure. The gauge as well as the fermion mass hierarchies are naturally generated in the same manner as in the extra-dimensional model, while also satisfying bonds from flavor physics and precision electroweak data. We also study a deconstructed model in which the Higgs is a pseudo Nambu-Goldstone boson, with the motivation of obtaining a naturally light and localized Higgs. 


\section{Agradecimentos}

Agradeço ao professor Gustavo Burdman, por sua orientação atenciosa e paciente, por ter me dado problemas interessantes em que trabalhar, que contribuiram muito para a minha formação e por ter acreditado em mim como estudante.

A todos os meus professores, que me motivaram e me ajudaram a subir alguns degraus dessa escada sem fim que é o conhecimento.

Aos amigos que fiz em todos esses anos na USP, pela companhia e conversas agradáveis, pelo apoio e pela ajuda nos momentos de necessidade. Em especial agradeço a Nayara por ter tido o trabalho de ler esta tese e me ajudar na correção ortográfica e na clareza do texto, e por garantir que o café de todas as tardes ocorresse no horário estabelecido.

Aos amigos de fora da USP, que me acompanham por quase toda a vida.

A minha família, pelo amor incondicional e por sempre me apoiarem no caminho que escolhi. Agradeço especialmente a minha mãe, Eliane, que se sacrificou muito para garantir que eu tivesse uma boa educação e pudesse seguir meus sonhos. Mesmo que ela não entenda o conteúdo, espero que quando tiver esse texto em mãos ela sinta que o seu esforço valeu a pena.

A Danuce que tem sido minha companheira e dividido comigo as alegrias e tristezas dessa vida de estudante, que ela entende tão bem quanto eu.

A FAPESP pelo apoio financeiro. 


\section{Conteúdo}

Resumo iii

$\begin{array}{lll}\text { Abstract } & \text { v }\end{array}$

Agradecimentos vii

1 Introdução 1

2 O Modelo de Randall-Sundrum 13

2.1 Introdução . . . . . . . . . . . . . . . . . . . 13

2.2 Bósons de Gauge . . . . . . . . . . . . . . . . . . 18

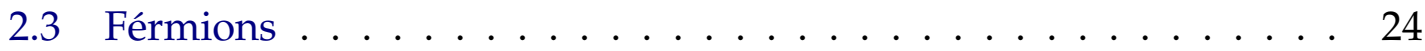

2.4 Acoplamentos e Violação de Sabor . . . . . . . . . . . . . . . . . 31

3 Desconstrução Dimensional 43

3.1 Introdução . . . . . . . . . . . . . . . . . . . . 43

3.2 Bósons de Gauge . . . . . . . . . . . . . . . . . . . 46

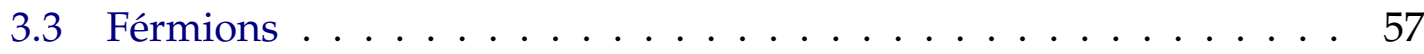

3.4 Acoplamentos . . . . . . . . . . . . . . . . . 66

3.5 Violação de Sabor . . . . . . . . . . . . . . . . . . . 71 
3.6 Parâmetros de Precisão Eletrofraca . . . . . . . . . . . . . . . . . . 81

4 O Higgs Como um Pseudo Bóson de Nambu-Goldstone 95

4.1 Motivação . . . . . . . . . . . . . . . . . . 95

4.2 O Modelo . . . . . . . . . . . . . . . . . . . . 101

4.3 Potencial Efetivo . . . . . . . . . . . . . . . . . . . . 110

5 Conclusões e Perspectivas 129

Bibliografia

130 


\section{Capítulo 1}

\section{Introdução}

O Modelo Padrão das interações fundamentais (MP) foi construído como uma teoria de gauge $S U(3)_{C} \times S U(2)_{L} \times U(1)_{Y}$, onde $S U(3)_{C}$ são as interações fortes e $S U(2)_{L} \times U(1)_{Y}$ é o setor eletrofraco, com simetrias de isospin fraco $S U(2)_{L}$ e hipercarga $U(1)_{Y}$. A matéria ocorre em três gerações de férmions: léptons que são singletos de $S U(3)_{C}$ e quarks que pertencem à representação fundamental. Todos os férmions do MP se dividem em dubletos de mão esquerda e singletos de mão direita sob $S U(2)_{L}$. O setor $S U(3)_{C}$ é responsável pelo confinamento dos quarks em hádrons. Já o setor eletrofraco $S U(2)_{L} \times U(1)_{Y}$ é descrito pela quebra espontânea de simetria $S U(2)_{L} \times U(1)_{Y} \rightarrow U(1)_{Q}$, onde $U(1)_{Q}$ é a simetria eletromagnética. Dessa maneira, os bósons de gauge responsáveis pelas interações fracas absorvem os bósons de Nambu-Goldstone da quebra e adquirem uma massa, tornando as interações fracas de curto alcance, enquanto que o fóton permanece com massa nula [1].

Até então, o MP tem sido extremamente bem sucedido em descrever as interações fundamentais e passou por testes de grande precisão [2, 3, 4, 5]. A última 
peça faltante prevista pelo MP é o setor de Higgs, responsável pela quebra da simetria eletrofraca (ElectroWeak Symmetry Breaking- EWSB). Com a descoberta de um novo bóson de massa $m_{H} \simeq 125 \mathrm{GeV}$ que tem grandes chances de ser o bóson de Higgs do MP [6, 7], parece que a física de partículas está completa. No entanto, o MP não pode ser considerado uma teoria final. Toda teoria física, e em particular as teorias quânticas de campos, possuem seu domínio de validade. No MP, o limite menos restritivo é imposto pela gravitação. Correções quânticas às equações de Einstein tornam-se importantes em energias da ordem da escala de Planck $\left(M_{P}=10^{19} \mathrm{GeV}\right)$, e invalidam a descrição consistente da gravitação como teoria quântica de campos, requerendo nova física, como por exemplo a teoria de cordas. ${ }^{1}$

Muito antes de atingir essa escala, existem outros problemas não contemplados pelo MP. Entre eles podemos citar a ausência de candidatos a matéria escura não bariônica, necessária para explicar as curvas de rotações de galáxias, a formação de estruturas do universo e o espectro das flutuações da radiação cósmica de fundo [9]. Também não há uma explicação natural para o valor medido da densidade de energia do vácuo $\rho_{v a c} \lesssim 10^{-46} \mathrm{GeV}^{4}$ ou, equivalentemente, da constante cosmológica [10,11], que acredita-se compor aproximadamente $70 \%$ da densidade de energia do universo e ser responsável por sua expansão acelerada.

De interesse particular para nós são os chamados problemas de hierarquia do $\mathrm{MP}$, pois eles sugerem a existência de nova física na escala $\mathrm{TeV}$, como veremos. No MP o setor de Higgs é representado por um dubleto escalar de $S U(2)_{L}$ com hipercarga 1/2. Embora tal escolha seja miníma, ela não pode ser considerada

\footnotetext{
${ }^{1}$ De fato, para energias $E \ll M_{P}$ é possível tratar a gravitação quântica como uma teoria de campos efetiva, de maneira análoga a descrição de Fermi das interações fracas, que funciona bem para $E \ll M_{E W} \sim 100 \mathrm{GeV}[8]$.
} 
satisfatória, pois escalares elementares sofrem do conhecido problema da hierarquia de escalas, ou hierarquia de gauge, descrito a seguir.

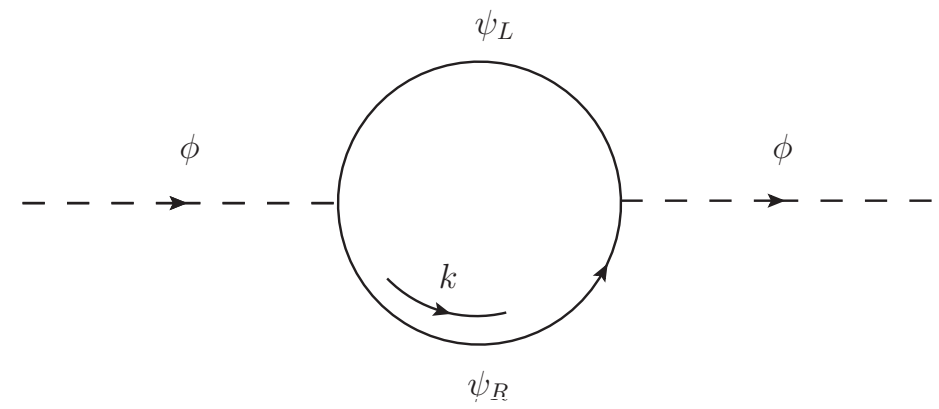

Figura 1.1: Contribuição a 1-loop para a massa do escalar $\phi$ devida a interação com o férmion $\psi$. O momento livre no loop é indicado por $k$.

Em teoria de perturbações, escalares sofrem correções radiativas à sua massa que divergem como o quadrado do corte de momento $(\Lambda)$ que define a escala de validade da teoria. Para ver isso, podemos considerar uma interação tipo Yukawa, $\mathcal{L}_{i n t}=g \bar{\psi}_{L} \phi \psi_{R}$ que contribui para a massa do escalar conforme é representado no diagrama da figura (1.1). Fazendo a análise dimensional, notamos que cada propagador fermiônico no loop contribui um fator $\sim 1 / k$ do momento livre no loop, que deve ser integrado, com um fator $d k^{4}$. A dimensão é então $k^{2}$, o que resulta em uma contribução para a massa $\delta m^{2} \sim \Lambda^{2}$, que é uma renormalização aditiva, quadraticamente divergente.

A mesma interação contribui para a massa de Dirac do férmion segundo o diagrama da figura (1.2). Fazendo a mesma análise, o propagador escalar contribui um fator $\sim 1 / k^{2}$, o fermiônico $\sim 1 / k$ e a integração $\sim d k^{4}$, portanto a dimensão de momento é $\sim k$. Em contraste com o caso escalar, vemos que para mudar a quiralidade do férmion $(R \rightarrow L)$ é necessária uma inserção da massa do férmion $m_{f}$, sendo assim, por análise dimensional as correções radiativas para a massa são da 


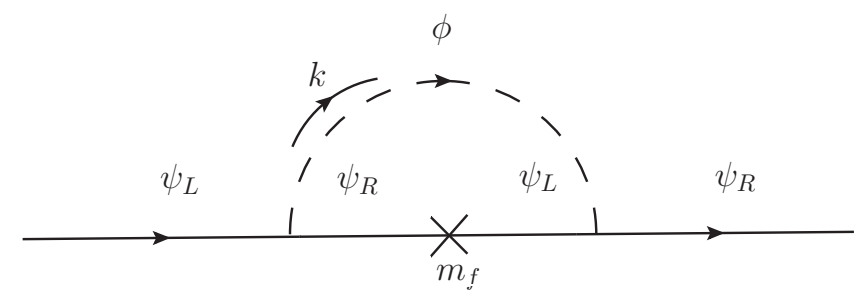

Figura 1.2: Contribuição a 1-loop para a massa do férmion $\psi$ devida a interação com o escalar $\phi$. O momento livre no loop é indicado por $k$ e a inserção da massa por $\times$.

forma $\delta m_{f} \sim m_{f} \ln \frac{\Lambda^{2}}{m_{f}^{2}}$. Considerações análogas se aplicam no caso de interações de gauge [1].

Se o Higgs é um escalar elementar, devido as correções quadráticas é natural que a sua massa seja da ordem do corte da teoria. No entanto, a teoria eletrofraca não é consistente a altas energias se a massa do bóson de Higgs for muito grande. O espalhamento de bósons longitudinais, por exemplo, no canal $W_{L}^{+} W_{L}^{-} \rightarrow W_{L}^{+} W_{L}^{-}$viola a unitariedade perturbativa a altas energias [12]. A teoria é unitarizada pelo bóson de Higgs, desde que sua massa esteja abaixo de $710 \mathrm{GeV}[12,13]$. Precisamos que a massa seja da ordem da escala eletrofraca $M_{E W}$, de centenas de GeVs, e assim, se assumirmos que o MP é válido até a escala de Planck, onde esperamos que os efeitos da gravitação quântica se tornem importantes, isso implica que necessitamos de um cancelamento finamente ajustado entre o parâmetro de massa nua do Higgs $m_{H 0}^{2}$ e a correção radiativa $\delta m_{H}^{2}$ :

$$
\begin{aligned}
M_{E W} / M_{P} & =10^{-17} ; \quad \delta m_{H}^{2} \approx M_{P}^{2} \Rightarrow \\
m_{H}^{2} & =m_{H 0}^{2}-\delta m_{H}^{2} \approx M_{E W}^{2} \Rightarrow \frac{m_{H 0}^{2}-\delta m_{H}^{2}}{m_{H 0}^{2}+\delta m_{H}^{2}} \simeq 10^{-34} .
\end{aligned}
$$

Esse cancelamento não é natural no sentido que não esperamos que a razão 
$M_{P} / M_{E W}$ seja tão pequena se não existe nenhuma simetria que proíba as correções radiativas quadráticas [14]. Em contraste, para férmions, existe a simetria quiral de rotações independentes para férmions de mão esquerda e direita. Essa simetria garante a proporcionalidade da correção radiativa com a massa do férmion, fazendo com que ela desapareça no limite $m_{f} \rightarrow 0$, que restaura a simetria, e seja logaritmicamente divergente, não sofrendo do problema de ajuste fino. A existência do problema da hierarquia é um argumento para supormos que exista nova física na escala $\mathrm{TeV}$ que elimine a necessidade do ajuste fino e permita obter assim um Higgs, ou seu equivalente, que seja naturalmente leve. De fato, se o corte do MP for $\Lambda \sim 1 \mathrm{TeV}$, vemos que a correção quadrática é de ordem $\delta m_{h}^{2} \sim(\Lambda / 4 \pi)^{2} \simeq(100 \mathrm{GeV})^{2}$, e não é necessário o ajuste fino.

A realidade do problema da hierarquia se torna mais clara se considerarmos uma analogia simples com um sistema de matéria condensada [1]. A energia livre de Gibbs em um sistema ferromagnético pode ser descrita na vizinhança da transição de fases por uma densidade de spins que é um campo escalar $\mathbf{s}(\mathbf{x})$ invariante por rotações $O(3)$ :

$$
G=\int d^{3} x\left[\frac{1}{2}(\nabla \mathbf{s})^{2}+b\left(T-T_{C}\right) \mathbf{s}^{2}+c \mathbf{s}^{4}+\mathbf{H} \cdot \mathbf{s}+\ldots\right]
$$

onde $\mathbf{H}$ é um campo magnético externo e $b$ e $c$ são números reais positivos. Esperamos que essa descrição macroscópica deixe de valer para um corte $\Lambda=1 / a$ onde $a$ é da ordem da distância interatômica. Se considerarmos os temos quadráticos em s, vemos que o análogo da massa do Higgs é o termo $b\left(T-T_{C}\right) \equiv 1 / 2 \xi^{2}$. Quando $T<T_{C}$, a "massa" torna-se negativa, e ocorre a quebra espontânea da simetria $O(3) \rightarrow O(2)$ (o material adquire uma orientação preferida, que dá a 
direção da magnetização macroscópica do imã ou do domínio magnético). Considerando $\mathbf{H}=0$, e desprezando termos de ordem superior, vemos que acima de $T_{C}$, a energia livre é minimizada para magnetização nula. O sistema é então paramagnético. Já, abaixo de $T_{C}$, temos magnetização não nula mesmo quando o campo externo é nulo, o sistema está na fase ferromagnética.

O parâmetro $\xi$ é uma medida das correlações entre os spins: $\langle\mathbf{s}(x) \mathbf{s}(y)\rangle \sim$ $e^{-r / \xi} / r$ (análogo ao comprimento de onda Compton no potencial de Yukawa). No ponto critico $T=T_{C}$, a transição de fases é de segunda ordem e os spins estarão correlacionados a distâncias macroscópicas, ou seja, $\xi \gg a$. O análogo disto para o campo de Higgs seria a condição $m_{H}^{2} \ll \Lambda^{2} \sim M_{P}^{2}$. Mas o sistema ferromagnético só atinge essa situação por um ajuste fino na temperatura, $T \approx T_{C}$. Enquanto podemos facilmente ajustar a temperatura em nosso arranjo experimental, não existe razão para supor que seja possível ajustar finamente a massa do campo de Higgs na natureza. Assim, concluímos que deveria existir um mecanismo capaz de explicar a condição $m_{H}^{2} \ll M_{P}^{2}$ sem grandes ajustes finos. Esse é o problema da hierarquia de gauge. ${ }^{2}$

Outro problema é a origem da hierarquia de massas dos férmions. No MP as massas dos férmions estão parametrizadas por acoplamentos de Yukawa que assumem valores que vão desde $\mathcal{O}(1)$ para o quark top até $\sim 10^{-6}$ para o elétron. Embora as massas dos férmions sejam naturais no sentido técnico (são protegidas pela simetria quiral), não existe nenhum mecanismo dinâmico no MP que gere essa grande hierarquia, e assim esperamos que a física além do MP seja ca-

\footnotetext{
${ }^{2} \mathrm{~A}$ conclusão depende da existência do corte $\Lambda$ como uma escala física, onde o MP deixa de ser consistente como teoria efetiva. Se assumirmos que o MP é válido até escalas arbitrariamente altas de energia, não há ajuste fino, pois o corte não é físico. No entanto como vimos isso não é razoável pois a gravitação impõe um corte $\Lambda \sim M_{P}$.
} 
paz de explicar satisfatoriamente esse espectro de massas que a baixas energias nos parece arbitrário. Da mesma forma, a matriz de mistura dos quarks também apresenta uma estrutura hierárquica (por exemplo, $V_{t b} \sim 1>>V_{t s}>>V_{t d}$ ), que do ponto de vista do MP não possui nenhuma explicação mais fundamental.

Resolver o problema da hierarquia de gauge requer nova física na escala $\mathrm{TeV}$, que torne o Higgs naturalmente leve, e em geral os graus de liberdade associados a essa física nova afetarão as medidas de precisão eletrofraca para energias da ordem dessa escala. Apesar disso, os dados tem confirmado as previsões do $\mathrm{MP}$, o que na linguagem de teorias efetivas implica que qualquer operador nãorenormalizável que se origine da física além do MP e afete as medidas de precisão estará suprimido por uma escala efetiva de vários $\mathrm{TeV}$. O conflito entre a necessidade de nova física para o setor de Higgs e sua aparente exclusão por medidas de precisão constitui o chamado "paradoxo" do LEP [15]. Nosso trabalho consiste em estudar possíveis soluções para os problemas de hierarquia do MP que respeitem os vínculos de precisão e da física de sabor, utilizando para isso a fenomenologia e a construção de modelos. Escolhemos como tema principal dessa tese nossos estudos sobre modelos inspirados na descontrução dimensional de teorias com dimensões extras curvas. Antes de apresentar esse tópico, faremos uma descrição breve de nossos outros trabalhos, que são também explorações da fenomenologia da física além do MP na escala TeV.

Qualquer medida que apresente uma discrepância com as previsões do MP, se levada a sério, pode ser um guia para apontar as características da nova física. Naturalmente, de antemão não sabemos se uma anomalia experimental é apenas um efeito estatístico que desaparecerá com o acumulo de mais dados ou se é um efeito genuíno além do MP. A assimetria forward-backward na produção 
Introdução

de pares de quarks top anti-top $\left(A_{F B}^{t}\right)$ foi recentemente medida no Tevatron e é um exemplo de observável que pode impor vínculos para a física nova. O experimento CDF obteve o resultado de $0.150 \pm 0.055$ no referencial do laboratório e $0.158 \pm 0.075$ no referencial partônico [16]. Subsequentemente, D0 reportou o resultado de $0.142 \pm 0.038$ e $0.196 \pm 0.065$ no laboratório e nível dos partons, respectivamente [17].

Frente a esse resultado, devemos nos perguntar qual a origem dinâmica dessa assimetria. No MP, sabemos que as interações fracas violam maximamente a paridade no acoplamento com os bósons $W$, que é puramente de mão esquerda. No entanto, surpreendentemente, as interações fortes também apresentam uma contribuição não nula para esse observável. A nível árvore a interação forte possui acoplamentos vetoriais e não apresenta, portanto, assimetria. No entanto cálculos a ordens mais altas de teoria de perturbação induzem uma assimetria não nula na produção de pares de quarks. Essa contribuição para a produção de pares top anti-top vinda do MP é estimada a NLO (next to leading order) através de métodos de Monte Carlo (MC@NLO) em $A_{F B}^{S M}=0.058 \pm 0.009$ [18] no referencial de repouso do par.

Os resultados do Tevatron são compatíveis com o MP, mas seu valor central está discrepante por no mínimo dois desvios padrões (ao considerar a distribuição da assimetria com a massa invariante do par de tops, a discrepância sobe para 3.4 desvios padrões para $M_{t \bar{t}}>450 \mathrm{GeV}$ no caso do CDF, por exemplo). Além disso, os excessos medidos pelo CDF e pelo D0 apontam para a mesma direção: uma assimetria grande e positiva. ${ }^{3}$ Frente a isso, podemos nos questionar

\footnotetext{
${ }^{3}$ Podemos nos perguntar se o LHC também confirma esse resultado, mas devido ao estado inicial simétrico $p p$ e ao grande fundo devido aos gluons, observáveis de assimetria são difíceis de medir [19, 20, 21], e a resposta ainda é inconclusiva.
} 
se não existe uma contribuição de nova física para esse observável.

Com essa motivação, estudamos a fenomenologia de uma nova interação forte na escala $\mathrm{TeV}$, que seria responsável por produzir $A_{f b}^{t \bar{t}}$. Esse modelo se insere na classe que busca obter a EWSB através de condensação de férmions fortemente acoplados, nesse caso uma quarta geração. No entanto, o resultado já é relevante apenas como um estudo do espaço de parâmetros de uma teoria que busque modelar esta assimetria através de um novo octeto de cor. Apresentamos um modelo fenomenológico de uma nova interação $S U(3)$ quebrada espontaneamente e fortemente acoplada na escala TeV. Essa interação produziria a condensação de uma quarta geração de quarks na escala $\mathrm{TeV}$, gerando assim a quebra espontânea da simetria eletrofraca de forma dinâmica. O estudo consistiu em determinar o espaço de parâmetros admissível para esse modelo sem que ele entrasse em conflito com vínculos impostos pela física de sabor e do quark top. Em particular, buscamos reproduzir os valores medidos da seção de choque de produção de pares top anti-top e da assimetria forward-backward, bem como as respectivas distribuições com a massa invariante do par. Também foi feita uma estimativa do sinal esperado da quarta geração no LHC.

Consideramos as massas $M_{G}=1 \mathrm{TeV}$ e $M_{G}=1.5 \mathrm{TeV}$ para o octeto de cor e buscamos os acoplamentos vetoriais e axiais deste com os quarks que permitissem cálculos perturbativos e satisfizessem os vínculos experimentais citados acima. Uma vez impostos esses vínculos, determinamos que a região favorecida do espaço de parâmetros é tal que o produto dos acoplamentos axiais do top e do quark do estado inicial é negativo. A assimetria do top é então gerada essencialmente pelo acoplamento de mão direita do top com a nova ressonância. Concluímos que é possível gerar a assimetria através de um octeto de cor com 
massa entre $M_{G}=1 \mathrm{TeV}$ e $M_{G}=1.5 \mathrm{TeV}$, em acordo com os vínculos experimentais da física de sabor e do top, para um acoplamento de mão direita do top com o novo estado com um valor entre $0<\left|g_{R}^{t}\right| \lesssim 2 \pi$ para $M_{G}=1 \mathrm{TeV}$ e $4 \lesssim\left|g_{R}^{t}\right| \lesssim 2 \pi$ para $M_{G}=1.5 \mathrm{TeV}$. Os resultados foram publicados e podem ser encontrados em [22].

Com a descoberta de uma nova ressonância no LHC, candidata a ser o bóson de Higgs, uma questão de grande relevância é determinar o quão compatíveis são as taxas de produção e razões de ramificação dessa partícula com as previstas para o Higgs do MP. Os dados mais recentes até a escrita deste texto são compatíveis com o MP. No entanto, no canal de decaimento do Higgs em dois fótons, existe um excesso que é aproximadamente duas vezes a taxa esperada do MP, com uma significância de dois sigma [6, 7]. Embora o Higgs não se acople diretamente ao fóton, esse canal é gerado por loops de partículas carregadas. Como o Higgs se acopla com uma intensidade proporcional à massa da partícula, no MP esse canal é dominado pelos loops do quark top e do bóson W, que interferem destrutivamente, produzindo uma largura de $\Gamma_{\gamma \gamma}^{S M} \simeq 0.008 \mathrm{MeV}$ (ver figura (1.3)). ${ }^{4}$ Experimentalmente, o que se observa é o produto da seção de choque de produção do Higgs (dominada pela fusão de glúons no LHC) vezes a razão de ramificação em fótons. A presença de novas partículas carregadas que recebem uma contribuição para a sua massa através do seu acoplamento com o Higgs afeta esse observável, e suas consequências tem sido exploradas na literatura recente $[23,24,25,26]$. Para modelos em que as novas partículas não possuem cor, e portanto, não afetam significativamente a taxa de produção, a razão de ramificação

\footnotetext{
${ }^{4} \mathrm{O}$ bóson $\mathrm{W}$ contribui com um diagrama adicional (não representado) devido ao acoplamento quártico $\sim g^{2} W^{+} W^{-} \gamma \gamma$.
} 

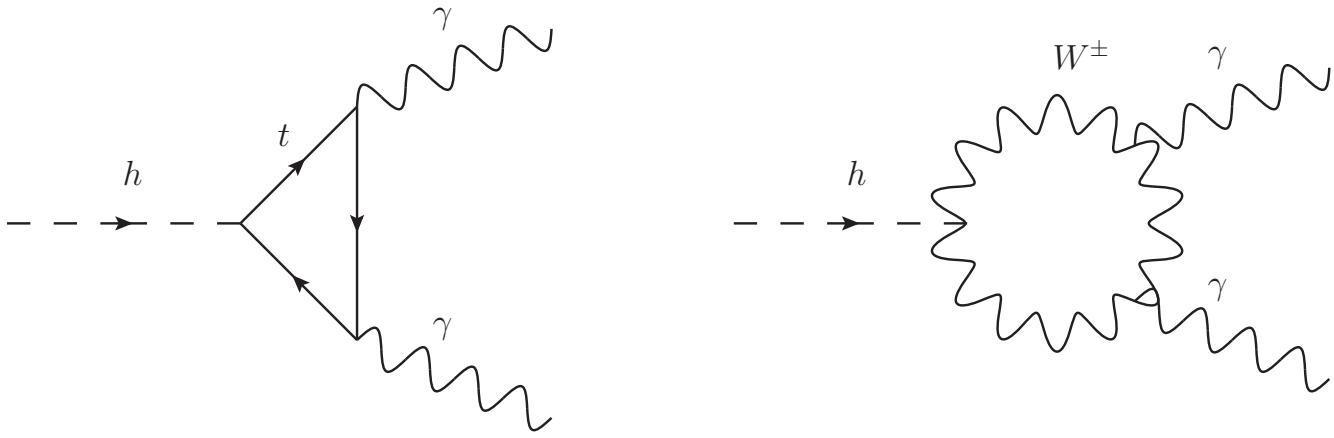

Figura 1.3: Contribuição a 1-loop para a largura do Higgs em dois fótons $\Gamma_{\gamma \gamma}^{S M}$. A esquerda (direita) temos a contribuição do quark top (bóson W). Os diagramas interferem destrutivamente.

em fótons é uma fonte importante de vínculos. Atualmente, estamos explorando o efeito das novas partículas sem cor previstas por modelos de física além do MP na modificação dos acoplamentos do Higgs para impor vínculos sobre esses modelos. Esse trabalho está atualmente em fase de preparação [27].

O tema que escolhemos desenvolver nesta tese diz respeito a construção de modelos da EWSB que são capazes de gerar a hierarquia de massas dos férmions e um Higgs leve, sem entrar em conflito com vínculos de precisão eletrofraca e de sabor, e que são inspirados em modelos com uma dimensão extra curva. O texto está estruturado da seguinte maneira: No segundo capítulo, faremos uma revisão do modelo de dimensões extras curvas de Randall-Sundrum (RS) [28], que é capaz de resolver os problemas de hierarquia de massas e de gauge, mas que para isso encontra problemas na física de sabor. No terceiro capítulo apresentaremos a ferramenta de construção de modelos chamada Desconstrução Dimensional $[29,30]$ e qual a sua relação com modelos de dimensões extras curvas e estudaremos um modelo particular, obtido pela aplicação dessa técnica ao modelo de RS, que é capaz de resolver os problemas de hierarquia de maneira similar a esse 
sem, no entanto, violar os vínculos de sabor. Esse estudo originou o trabalho [31]. No quarto capítulo apresentaremos os resultados obtidos até o momento sobre o setor de Higgs. Nesse tipo de modelo, esperamos obter o Higgs como um pseudo bóson de Nambu-Goldstone, o que o torna naturalmente leve. Esse trabalho está em fase de preparação [32]. Finalmente, apresentamos nossas conclusões. 


\section{Capítulo 2}

\section{O Modelo de Randall-Sundrum}

\subsection{Introdução}

Teorias com dimensões extras fornecem uma ferramenta de contrução de modelos que permite solucionar alguns dos problemas do MP (ver, por exemplo, [33]) e geram uma fenomenologia interessante. Faremos aqui uma breve descrição do modelo de dimensões extras curvas (Warped Extra Dimensions - WED) de Randall-Sundrum (RS) [28, 34], e de como ele é capaz de resolver os problemas da hierarquia de gauge e das massas dos férmions. Por analogia a esse modelo chegaremos no capítulo (3) ao modelo de Descontrução Dimensional que nos interessa. Nosso tratamento do presente tema é baseado nas referências $[35,36,37,38,39]$.

O modelo de RS é formulado em um fundo dado por uma métrica anti-de Sitter em cinco dimensões $\left(\operatorname{Ad} S_{5}\right)$, que pode ser escrita:

$$
d s^{2}=g_{M N} d x^{M} d x^{N}=e^{-2 k y} \eta_{\mu \nu} d x^{\mu} d x^{\nu}-d y^{2},
$$


onde $y$ é a coordenada da dimensão extra, $k$ é a curvatura constante e $\eta_{\mu \nu}=$ $\operatorname{diag}(1,-1,-1,-1)$ é a métrica de Minkowski em quatro dimensões. Convencionamos que índices gregos referem-se as coordenadas quadridimensionais $(\mu, \nu=$ $0,1,2,3)$ e indices latinos capitalizados referem-se a todas as cinco coordenadas $(M, N=0,1,2,3,5)$. Para cada valor fixo de $y$, temos um sub-espaço plano de Minkowski (3-brana).

Para escapar vínculos de detecção (por exemplo, mudanças mensuráveis no potencial gravitacional estático) a dimensão extra deve ser compacta e muito pequena. ${ }^{1}$ A escolha mais simples é tomarmos a dimensão extra compactificada em um círculo de raio $R\left(S^{1}\right){ }^{2}$ Tomamos $-\pi R \leq y<\pi R$ e portanto os campos devem satisfazer condições de contorno periódicas:

$$
\Phi(x, y+2 \pi R)=\Phi(x, y)
$$

Veremos adiante que o fato da dimensão extra ser compacta implica que a dependência em $y$ dos campos pode ser expressa em um conjunto completo de modos ortonormais, chamados modos de Kaluza-Klein (KK). Os modos de energia mais baixa, chamados modos zero, corresponderão às partículas do MP no ponto de vista da teoria efetiva quadridimensional obtida pela integração da dimensão extra.

Em alguns casos, não é desejável que o campo cinco dimensional possua um modo zero, pois ele não é observado no espectro do MP. Como exemplo, temos a quinta componente de um campo de gauge, $A_{5}$, que se transformaria como um

\footnotetext{
${ }^{1}$ Na realidade, o modelo de $W E D$ em $A d S_{5}$ admite uma dimensão extra infinita, o chamado modelo RSII [34], mas não estamos interessados nesse limite.

${ }^{2}$ É necessário um mecanismo para estabilizar dinamicamente o raio da dimensão extra [36, 40]. Não trataremos desse problema aqui.
} 
escalar sem massa sob a simetria de Lorentz quadridimensional. Um mecanismo que permite eliminar os modos zero indesejáveis é o chamado orbifolding, que consiste em compactificar a dimensão extra em um orbifold $S^{1} / \mathbb{Z}_{2}$. A simetria discreta $\mathbb{Z}_{2}$ corresponde a transformação $y \rightarrow-y$, de maneira que os pontos $y$ e $-y$ do círculo são identificados (ver figura (2.1)). Assim, a coordenada $y$ varia de $y=0$ até $y=\pi R$. Os pontos $y=0$ e $y=\pi R$ são pontos fixos, invariantes sob $\mathbb{Z}_{2}$. Se tomarmos os campos cinco dimensionais com paridade $\mathbb{Z}_{2}$ definida, então teremos a liberdade de escolher quais campos terão um modo zero. Em particular, isso permitirá obter férmions quirais, como veremos.

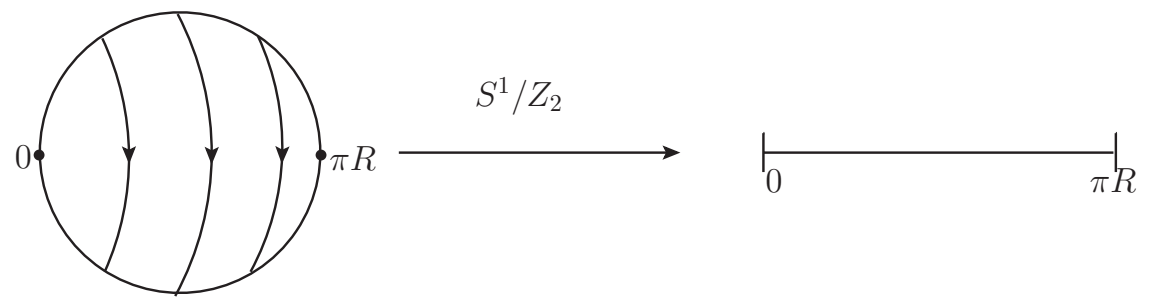

Figura 2.1: Compactificação em orbifold.

Chamaremos as branas localizadas nos pontos $y=0$ e $y=\pi R$ de branas UV (ou Planck) e IR (ou TeV), respectivamente. ${ }^{3} \mathrm{O}$ espaço entre as branas UV e IR é chamado de bulk. Pode-se mostrar que a solução dada pela métrica $A d S_{5}$ implica que o campo do gráviton (modo zero) está exponencialmente localizado na brana UV $[28,40]$ :

$$
h_{\mu \nu}(x, y)=e^{-2 k y} \bar{h}_{\mu \nu}(x) .
$$

A intensidade da interação gravitacional efetiva vista por um observador qua-

\footnotetext{
${ }^{3}$ Por brana, entendemos o subespaço plano quadridimensional localizado nos pontos fixos do orbifold. Não associamos a essas branas uma dinâmica, seu papel será apenas de impor condições de contorno aos campos.
} 
dridimensional pode ser obtida integrando a ação sobre a dimensão extra. ${ }^{4}$ Claramente, a maior contribuição virá de $y=0$. Assim, observadores localizados no UV sentirão uma interação gravitacional forte, enquanto que observadores localizados no IR perceberão a gravidade como uma força fraca.

De fato, a métrica (2.1) é invariante sob a transformação:

$$
\begin{aligned}
& y \rightarrow y+\lambda \\
& x \rightarrow e^{k \lambda} x .
\end{aligned}
$$

Dessa forma, vemos que deslocamentos em $y$ correspondem a um aumento das escalas de distância por um fator exponencial (fator de warping), ou, equivalentemente, todas as escalas de energia diminuem exponencialmente $\left(\partial_{\mu} \rightarrow\right.$ $\left.e^{-k \lambda} \partial_{\mu}\right)$. Associaremos à brana UV uma escala de energia de ordem $M_{P}=10^{19} \mathrm{GeV}$. Se escolhermos $k \pi R \simeq 37$ (correspondendo a um raio da dimensão extra de or$\operatorname{dem} R \gtrsim 10 L_{P}$, com $L_{P} \equiv M_{P}^{-1}$ o comprimento de Planck, para $\left.k \lesssim M_{P}\right)$, vemos que a correspondente escala de energia da brana IR será suprimida por um fator:

$$
M_{I R}=e^{-k \pi R} M_{P} \simeq 10^{-16} M_{P} \simeq 1 \mathrm{TeV} .
$$

Isso permite resolver o problema da hierarquia de gauge, pois o valor esperado de vácuo do Higgs também será naturalmente suprimido pelo warping, desde que o Higgs esteja localizado próximo de $y=\pi R$. Podemos ver esse me-

\footnotetext{
${ }^{4}$ De fato, se a escala de energia fundamental da gravitação for $M *$, integrando sobre a dimensão extra obtemos a escala quadridimensional $M_{P}^{2}=\frac{M *^{3}}{k}\left[1-e^{-2 k \pi R}\right.$ [ [28], que não é muito sensível ao tamanho da dimensão extra. Assim, estamos assumindo $k \lesssim M * \sim M_{P}$, com a curvatura suficientemente pequena para que a descrição clássica da gravitação ainda seja uma boa aproximação.
} 
canismo atuar explicitamente se assumirmos que o campo de Higgs esteja localizado na brana IR por uma função delta:

$$
S_{H}=\int d^{4} x \int_{0}^{\pi R} d y \sqrt{g} \delta(y-\pi R)\left[g^{\mu \nu} \partial_{\mu} H^{\dagger} \partial_{\nu} H-\lambda\left(|H|^{2}-v_{0}^{2}\right)^{2}\right]
$$

onde $g \equiv\left|\operatorname{det}\left(g_{M N}\right)\right|=e^{-4 k y}$. Podemos obter a ação efetiva quadridimensional do Higgs efetuando a integral em $y$ :

$$
S_{H}=\int d^{4} x\left[e^{-2 k \pi R} \eta^{\mu \nu} \partial_{\mu} H^{\dagger} \partial_{\nu} H-e^{-4 k \pi R} \lambda\left(|H|^{2}-v_{0}^{2}\right)^{2}\right] .
$$

O termo cinético pode ser canonicamente normalizado se redefinirmos:

$$
e^{-k \pi R} H \rightarrow H \text {. }
$$

Obtemos assim a ação:

$$
S_{H}=\int d^{4} x\left[\eta^{\mu \nu} \partial_{\mu} H^{\dagger} \partial_{\nu} H-\lambda\left(|H|^{2}-e^{-2 k \pi R} v_{0}^{2}\right)^{2}\right]
$$

O valor esperado de vácuo efetivo do Higgs será dado por $v \equiv e^{-k \pi R} v_{0}$. De fato, temos uma solução para o problema da hierarquia, pois mesmo que o vev (valor esperado de vácuo) do Higgs seja da ordem de $M_{P}$, sua localização na brana IR implica que um observador quadridimensional verá um vev exponencialmente suprimido, de ordem TeV. É claro que localizar o Higgs utilizando uma função delta é apenas um artifício para demonstrar a supressão, de modo que modelos mais realistas necessitam obter um mecanismo dinâmico de localização. 
Concluímos esta introdução comentando que modelos de WED possuem uma descrição dual quadridimensional através da correspondência $A d S / C F T$ [41]. Eles podem ser vistos como descrevendo uma teoria quadridimensional fortemente acoplada com dinâmica quase conforme, que vive na fronteira do espaço $A d S_{5}$. Um review dessa chamada descrição holográfica pode ser encontrado em $[37,38]$. O espectro de modos de KK corresponde a estados compostos na teoria quadridimensional, enquanto que os modos zero podem ser vistos como estados compostos ou elementares, dependendo de sua localização na dimensão extra. Assim, do ponto de vista observacional, não existiria distinção entre modelos com dimensões extras curvas e modelos quadridimensionais de quebra dinâmica de simetria através de interações fortes quase conformes, como modelos de Walking Technicolor [42]. Essa visão é útil para obter intuição sobre como contruir modelos realistas fortemente acoplados, além de servir como um método de cálculo poderoso para estes.

Passamos agora a obter o espectro de modos de KK para campos de gauge e férmions em cinco dimensões.

\subsection{Bósons de Gauge}

Vamos obter a teoria efetiva quadridimensional de modos de KK para um campo de gauge em um fundo $A d S_{5}$. Por simplicidade, trateremos o caso de uma simetria de calibre $U(1)$. A ação cinco dimensional é dada por: 


$$
\begin{aligned}
S_{5}^{A} & =\int d^{4} x \int_{0}^{\pi R} d y \sqrt{g}\left\{-\frac{1}{4} F_{M N} F^{M N}\right\} \\
& =\int d^{4} x \int_{0}^{\pi R} d y\left\{-\frac{1}{4} F_{\mu \nu} F^{\mu \nu}+\frac{1}{2} e^{-2 k y}\left(\partial_{5} A_{\mu}-\partial_{\mu} A_{5}\right)^{2}\right\}
\end{aligned}
$$

onde $\partial_{5} \equiv \partial_{y}$ e o tensor intensidade do campo é dado por $F_{M N}=\partial_{M} A_{N}-\partial_{N} A_{M}$. Na segunda linha, as contrações são feitas com a métrica de Minkowski $\eta_{\mu \nu}$. Vamos tomar o campo $A_{\mu}$ como par sob a simetria $\mathbb{Z}_{2}$. Primeiramente vemos que isso implica que a quinta componente $A_{5}$ tem de ser ímpar, para que o segundo termo da ação tenha paridade bem definida. Campos pares possuirão modo zero e campos ímpares não [35], o que mostra que o orbifold serve para projetar fora modos zero não desejados.

A paridade implica também que as condições de contorno que os campos obedecem devem ser do tipo Neumann, para campos pares ou Dirichlet, para os ímpares, ou seja:

$$
\begin{gathered}
\left.\partial_{5} A_{\mu}\left(x^{\mu}, y\right)\right|_{y=0}=\left.\partial_{5} A_{\mu}\left(x^{\mu}, y\right)\right|_{y=\pi R}=0, \\
\left.A_{5}\left(x^{\mu}, y\right)\right|_{y=0}=\left.A_{5}\left(x^{\mu}, y\right)\right|_{y=\pi R}=0 .
\end{gathered}
$$

Para poder efetuar a integral em $y$ e obter a ação efetiva quadridimensional, introduzimos as expansões em modos de KK:

$$
A_{\mu}\left(x^{\mu}, y\right) \equiv \frac{1}{\sqrt{\pi R}} \sum_{n=0}^{\infty} f^{(n)}(y) A_{\mu}^{(n)}\left(x^{\mu}\right)
$$




$$
A_{5}\left(x^{\mu}, y\right) \equiv \frac{1}{\sqrt{\pi R}} \sum_{n=0}^{\infty} \frac{\partial_{5} f^{(n)}(y)}{m_{n}} A_{5}^{(n)}\left(x^{\mu}\right)
$$

As $f^{(n)}(y)$ são chamadas "funções de onda" na dimensão extra. Para que $A_{\mu}$ seja par sob $\mathbb{Z}_{2}$ elas tem de satisfazer:

$$
f^{(n)}(y)=f^{(n)}(-y),\left.\quad \partial_{5} f^{(n)}(y)\right|_{0, \pi R}=0
$$

E sua normalização é dada por:

$$
\frac{1}{\pi R} \int_{0}^{\pi R} f^{(n)}(y) f^{(m)}(y) d y=\delta^{n m} .
$$

Veremos que as funções de onda $f^{(n)}(y)$ são um conjunto completo de soluções que diagonalizam a equação de movimento dos campos, e assim os estados da torre de modos de KK terão massas bem definidas. O termo de mistura na ação entre $A_{\mu}$ e $A_{5}$ pode ser eliminado indo ao gauge $A_{5}=0$. Esse gauge é análogo ao gauge unitário escolhido para eliminar modos de NG no mecanismo de Higgs. ${ }^{5}$ De fato, os modos de KK de $A_{5}$ se comportam como escalares sob a simetria de Lorentz quadridimensional e podem ser vistos como bósons de NG, que são absorvidos para dar massa aos modos de KK de $A_{\mu}$ [36]. Nesse gauge, as equações de movimento de $A_{\mu}$ se escrevem:

$$
\left[-\partial^{\nu} \partial_{\mu}+\delta_{\mu}^{\nu}\left(\partial^{2}-\partial_{5} e^{2 k y} \partial_{5}\right)\right] A^{\mu}=0
$$

onde $\partial^{2} \equiv \eta^{\mu \nu} \partial_{\mu} \partial_{\nu}$. Todas as contrações são feitas com $\eta_{\mu \nu}$. Introduzindo a ex-

\footnotetext{
${ }^{5}$ De maneira mais cuidadosa, introduzimos a classe de gauges $R_{\xi}$ através do termo de fixação de gauge $\mathcal{L}_{G}=-\sqrt{g} / \xi \operatorname{Tr}\left[D^{M} A_{M}^{2}\right]$, com $D_{M} A^{N}=\partial_{M} A^{N}+\Gamma_{M, P}^{N} A^{P}+i g\left[A_{M}, A^{N}\right]$. Podemos então desacoplar $A_{5}$ no limite $\xi \rightarrow \infty[43,44]$.
} 
pansão de modos de KK, obtemos:

$$
\left(\delta_{\nu}^{\mu} \partial^{2}-\partial^{\mu} \partial_{\nu}\right) A^{\nu(n)} f^{(n)}(y)-\partial_{5}\left(e^{2 k y} \partial_{5} f^{(n)}(y)\right) A^{\mu(n)}=0 .
$$

Para obter modos de KK com massas definidas, eles devem satisfazer:

$$
\left(\delta_{\nu}^{\mu} \partial^{2}-\partial^{\mu} \partial_{\nu}\right) A^{\nu(n)}=m_{n}^{2} A^{\mu(n)}
$$

Tomando a divergência $\partial_{\mu}$ dessa equação, vemos que ela implica a condição de Lorenz, $\partial_{\mu} A^{\mu(n)}=0$, ou seja, os modos de KK satisfazem equações de Proca:

$$
\left(\partial^{2}+m_{n}^{2}\right) A_{\mu}^{(n)}=0
$$

A equação de movimento que determina as funções de onda fica então:

$$
\partial_{5}^{2} f^{(n)}(y)-2 k \partial_{5} f^{(n)}(y)+m_{n}^{2} e^{2 k y} f^{(n)}(y)=0
$$

No caso não-abeliano, a diagonalização da parte quadrática da ação é idêntica, resultando no mesmo perfil na dimensão extra. No entanto, as interações de três e quatro campos de gauge que vem do termo cinético de Yang-Mills não são diagonalizadas por essa transformação, resultando em interações entre os modos de KK [30].

Podemos obter a solução para o modo zero $m_{0}=0$ :

$$
\partial_{5}^{2} f^{(0)}(y)-2 k \partial_{5} f^{(0)}(y)=0
$$


A solução geral é da forma:

$$
f^{(0)}(y)=A e^{2 k y}+B .
$$

com $A$ e $B$ constantes arbitrárias. As condições de contorno de Neumann (2.11) implicam que $A=0$. A normalização (2.16) dá então:

$$
f^{(0)}(y)=1
$$

O modo zero de um campo de gauge tem portanto um perfil plano na dimensão extra, garantindo universalidade dos seus acoplamentos. Isso é esperado, uma vez que o modo zero não possui massa, e assim, a simetria de gauge associada a ele não é quebrada.

Para obter a solução geral de (2.21) para todo $n$, definimos:

$$
z \equiv \frac{m_{n} e^{k y}}{k}, \quad f^{(n)}(z) \equiv z F^{(n)}(z)
$$

Substituindo essas definições em (2.21), obtemos:

$$
\begin{aligned}
& z^{2} \partial_{z}^{2} f^{(n)}-z \partial_{z} f^{(n)}+z^{2} f^{(n)}= \\
& z^{2} \partial_{z}^{2} F^{(n)}+z \partial_{z} F^{(n)}+\left(z^{2}-1\right) F^{(n)}=0 .
\end{aligned}
$$

Vemos que $F^{(n)}(z)$ satisfaz uma equação de Bessel de ordem $\nu=1$, e assim, podemos escrever a solução de (2.21) em termos de funções de Bessel e Neumann [35]:

$$
f^{(n)}(y)=\frac{e^{k y}}{N_{n}}\left[J_{1}\left(\frac{m_{n} e^{k y}}{k}\right)+\beta\left(m_{n}\right) Y_{1}\left(\frac{m_{n} e^{k y}}{k}\right)\right]
$$


onde $\beta\left(m_{n}\right)$ e $m_{n}$ são constantes e podem ser determinados pelas condições de contorno (2.11) e $N_{n}$ pela normalização (2.16).

A condição de contorno em $y=0$ dá:

$$
\begin{gathered}
{\left.\left[z\left(J_{1}(z)+\beta\left(m_{n}\right) Y_{1}(z)\right)+z^{2}\left(\partial_{z} J_{1}(z)+\beta\left(m_{n}\right) \partial_{z} Y_{1}(z)\right)\right]\right|_{y=0}=0} \\
\left.\Rightarrow\left[z^{2}\left(J_{0}(z)+\beta\left(m_{n}\right) Y_{0}(z)\right)\right]\right|_{y=0}=0
\end{gathered}
$$

onde usamos a relação de recorrência (válida para $J_{n}(x)$ e $Y_{n}(x)$ ):

$$
J_{n}^{\prime}(x)+\frac{n}{x} J_{n}(x)=J_{n-1}(x) .
$$

A constante $\beta\left(m_{n}\right)$ vale então:

$$
\beta\left(m_{n}\right)=-\frac{J_{0}\left(\frac{m_{n}}{k}\right)}{Y_{0}\left(\frac{m_{n}}{k}\right)} .
$$

O espectro de massas pode ser determinado grafica ou numericamente da equação transcendental dada pela condição de contorno em $y=\pi R$ :

$$
Y_{0}\left(\frac{m_{n}}{k}\right) J_{0}\left(\frac{m_{n} e^{k \pi R}}{k}\right)-J_{0}\left(\frac{m_{n}}{k}\right) Y_{0}\left(\frac{m_{n} e^{k \pi R}}{k}\right)=0
$$

No limite $m_{n} \ll k$ e $k R \gg 1$ podemos aproximar a constante de normalização por [35]:

$$
N_{n} \simeq \frac{e^{\pi k R} \pi R}{g_{5}^{2} \sqrt{2 \pi k R}} J_{1}\left(\frac{m_{n} e^{\pi k R}}{k}\right) \simeq \frac{e^{\pi k R / 2}}{g_{5}^{2}} \sqrt{\frac{R}{m_{n}}}
$$


Nesse limite, o espectro de massas é dado por:

$$
m_{n} \simeq\left(n-\frac{1}{4}\right) \pi k e^{-\pi k R}, \quad n>0
$$

Vemos que mesmo para uma curvatura grande, se aproximando de $M_{P}$, a presença do fator de warping gera um espectro de modos massivos com espaçamento de ordem TeV. Iremos agora descrever o espectro de férmions do modelo.

\subsection{Férmions}

A ação fermiônica em cinco dimensões pode ser escrita como:

$$
S_{5}^{f}=\int d^{4} x \int_{0}^{\pi R} d y \sqrt{g}\left\{i \bar{\Psi} \Gamma^{M} \nabla_{M} \Psi+m_{\Psi} \bar{\Psi} \Psi\right\}
$$

Para acoplar os espinores ao campo gravitacional, é necessário considerar sua transformação sob uma simetria de Lorentz local. Pelo princípio da equivalência, dado um ponto do espaço-tempo, sempre é possível encontrar um conjunto de coordenadas tal que a métrica nesse ponto seja a métrica de Minkowski. Isso permite definir referenciais ortonormais em cada ponto do espaço-tempo, com auxílio do chamado vielbein $e^{A}{ }_{M} \cdot{ }^{6}$ Em termos do vielbein, a métrica se escreve:

$$
g_{M N}=e^{A}{ }_{M} e^{B}{ }_{N} \eta_{A B},
$$

onde $\eta_{A B}$ é a métrica de Minkowski. Convencionamos que os índices do começo

\footnotetext{
${ }^{6}$ Do alemão, muitas pernas. Em quatro dimensões, usa-se o nome vierbein (quatro pernas).
} 
do alfabeto $(A, B, \ldots)$ se referem a transformações de Lorentz locais, enquanto que os índices $(M, N, \ldots)$ se referem a transformações gerais de coordenadas. $\mathrm{O}$ vielbein inverso pode ser obtido fazendo:

$$
E_{A}^{M}=g^{M N} \eta_{A B} e^{B}{ }_{N}
$$

E satisfaz:

$$
\begin{aligned}
e^{A}{ }_{M} E_{B}{ }^{M} & =\delta_{B}^{A}, \\
E_{A}{ }^{M} e^{A}{ }_{N} & =\delta_{N}^{M} .
\end{aligned}
$$

As matrizes $\Gamma^{M}(x, y)$ satisfazem a algebra de Clifford:

$$
\left\{\Gamma^{M}, \Gamma^{N}\right\}=2 g^{M N}
$$

e podem ser escritas como:

$$
\Gamma^{M} \equiv E_{A}^{M} \gamma^{A}
$$

onde as matrizes $\gamma^{A}=\left(\gamma^{\mu}, i \gamma^{5}\right), \gamma^{5} \equiv i \gamma^{0} \gamma^{1} \gamma^{2} \gamma^{3}$ são escolhidas para satisfzer:

$$
\left\{\gamma^{A}, \gamma^{B}\right\}=2 \eta^{A B}
$$

Finalmente, a derivada covariante espinorial é escrita:

$$
\nabla_{M}=\partial_{M}+\omega_{M}
$$

O campo $\omega_{M} \equiv i \omega_{A B M} \frac{\sigma^{A B}}{2}$ é a chamada conexão de spin, que se transforma 
como um campo de gauge sob transformações locais de Lorentz (grupo de gauge $\mathrm{SO}(1,4))$. Ela é composta dos geradores do grupo de Lorentz na representação espinorial: $\sigma^{A B} \equiv \frac{i}{4}\left[\gamma^{A}, \gamma^{B}\right]$ e da conexão de Levi-Civita $\Gamma_{M O}^{N}$ (que não deve ser confundida com a matriz de Dirac $\Gamma^{M}$ ) expressa na base ortonormal: $\omega_{A B M}=-g_{M N} E_{B}^{M}\left(\partial_{M} E_{A}^{N}+\Gamma_{M O}^{N} E_{A}^{O}\right)$. Para o caso específico da métrica $A d S_{5}$ (2.1), temos:

$$
\begin{aligned}
\Gamma_{\mu \nu}^{5} & =-k e^{-2 k y} \eta_{\mu \nu}, \Gamma_{5 \nu}^{\mu}=-k \delta_{\nu}^{\mu} \\
E_{A}^{M} & =\operatorname{diag}\left(e^{k y}, e^{k y}, e^{k y}, e^{k y}, 1\right) \\
\omega_{M} & =\left(\frac{k}{2} e^{-k y} i \gamma_{5} \gamma_{\mu}, 0\right) .
\end{aligned}
$$

Com essas definições, podemos escrever a ação como:

$$
S_{5}^{f}=\int d^{4} x \int_{0}^{\pi R} d y e^{-4 k y}\left\{e^{k y} i \bar{\Psi} \gamma^{\mu} \partial_{\mu} \Psi+\bar{\Psi}\left(2 k \gamma^{5}-\gamma^{5} \partial_{5}\right) \Psi+m_{\Psi} \bar{\Psi} \Psi\right\}
$$

Como $\gamma^{5}$ foi incluído na álgebra de Clifford, e não existe outra matriz que anticomute com as outras matrizes gama, não existem espinores quirais em cinco dimensões (por essa razão, o termo de massa para os férmions não é proibido por simetrias de gauge). No entanto, ainda podemos efetuar a decomposição $\Psi_{L, R}=\frac{\left(1 \mp \gamma^{5}\right)}{2} \Psi$, chegando à expressão:

$$
\begin{array}{r}
S_{5}^{f}=\int d^{4} x \int_{0}^{\pi R} d y e^{-4 k y}\left\{e^{k y} i \bar{\Psi}_{L, R} \gamma^{\mu} \partial_{\mu} \Psi_{L, R}+\left(\bar{\Psi}_{L}\left(2 k-\partial_{5}\right) \Psi_{R}-L \leftrightarrow R\right)+\right. \\
\left.+m_{\Psi}\left(\bar{\Psi}_{L} \Psi_{R}+\text { h.c. }\right)\right\} .
\end{array}
$$


Vemos que para que o termo com $\partial_{5}$ seja invariante sob $\mathbb{Z}_{2}, \Psi_{L}$ e $\Psi_{R}$ devem ter paridades opostas, ou seja, $\Psi(y)= \pm \gamma^{5} \Psi(-y) .{ }^{7}$ O campo que escolhermos como par terá modo zero, e o outro não. Isso permite obter um modo zero quiral, embora os acoplamentos de gauge cinco dimensionais sejam necessariamente vetoriais. Novamente, vemos que a compatificação em um orbifold elimina um modo zero indesejado.

Por outro lado, $\bar{\Psi} \Psi$ é ímpar sob $\mathbb{Z}_{2}$, o que proíbiria a massa para o férmion. No entanto, podemos supor que a massa se originou de um valor esperado de vácuo de um campo escalar no bulk com um perfil ímpar na dimensão extra, gerando um termo de massa da forma [35]:

$$
\begin{aligned}
m_{\Psi} & =c k \epsilon(y), \\
\epsilon(y) & \equiv \frac{y}{|y|},
\end{aligned}
$$

onde $c$ é um parâmetro arbitrário, de ordem um. Notamos que a parametrização $m_{\Psi}=c k$ é tecnicamente natural, uma vez que a massa será da ordem da escala de energia intrínseca da teoria, $k \lesssim M_{P}$. Um termo de massas construído assim é ímpar sob $\mathbb{Z}_{2}$, e portanto é permitido. Ele também irá modificar as condições de contorno de Neumann obedecidas pelos modos pares [35, 37], pois gera termos de massa com sinais opostos nas branas $\left.m_{\Psi}\right|_{0, \pi R}= \pm c k$, que devem ser cancelados.

É útil definir: $\tilde{\Psi}_{L, R} \equiv e^{-2 k y} \Psi_{L, R}$. Em termos desses campos, escrevemos a ação

\footnotetext{
${ }^{7}$ Notamos que ainda resta o termo proibido $2 k \bar{\Psi}_{L} \Psi_{R}$. Um tratamento mais cuidadoso requer tomar o fator de warping $e^{-2 k|y|}, 0<y<2 \pi R$. Assim, as derivadas em $y$ atuando na exponencial serão descontínuas em $y=0, \pi R$, gerando termos com funções degrau invariantes sob $\mathbb{Z}_{2}$, assim como na equação $(2.45)$ [36, 35].
} 
(2.44) como:

$$
\begin{array}{r}
S_{5}^{f}=\int d^{4} x \int_{0}^{\pi R} d y\left\{e^{k y} i \overline{\tilde{\Psi}}_{L, R} \gamma^{\mu} \partial_{\mu} \tilde{\Psi}_{L, R}-\right. \\
\left(\overline{\tilde{\Psi}}_{L} \partial_{5} \tilde{\Psi}_{R}-L \leftrightarrow R\right)+ \\
\left.+m_{\Psi}\left(\overline{\tilde{\Psi}}_{L} \tilde{\Psi}_{R}+\text { h.c. }\right)\right\} .
\end{array}
$$

Obtemos dessa ação as equações de movimento acopladas para os campos $L$ e $R$ :

$$
\begin{aligned}
e^{k y} i \gamma^{\mu} \partial_{\mu} \tilde{\Psi}_{L}-\partial_{5} \tilde{\Psi}_{R}+c k \tilde{\Psi}_{R} & =0 \\
e^{k y} i \gamma^{\mu} \partial_{\mu} \tilde{\Psi}_{R}+\partial_{5} \tilde{\Psi}_{L}+c k \tilde{\Psi}_{L} & =0 .
\end{aligned}
$$

Introduzimos aqui as expansões em modos de KK:

$$
\tilde{\Psi}_{L, R} \equiv \frac{1}{\sqrt{\pi R}} \sum_{n=0}^{\infty} h_{L, R}^{(n)}(y) \psi_{L, R}^{(n)}\left(x^{\mu}\right) .
$$

Normalizamos as funções de onda como:

$$
\frac{1}{\pi R} \int_{0}^{\pi R} e^{k y} h_{L, R}^{*(n)}(y) h_{L, R}^{(m)}(y) d y=\delta^{n m} .
$$

Substituindo em (2.47) e impondo que os modos de KK satisfaçam a equação de Dirac:

$$
i \gamma^{\mu} \partial_{\mu} \psi^{(n)}-m_{n} \psi^{(n)}=0
$$

Obtemos as equações para as funções de onda:

$$
\begin{aligned}
e^{k y} m_{n} h_{L}^{(n)}-\partial_{5} h_{R}^{(n)}+c k h_{R}^{(n)} & =0 \\
e^{k y} m_{n} h_{R}^{(n)}+\partial_{5} h_{L}^{(n)}+c k h_{L}^{(n)} & =0 .
\end{aligned}
$$


Elas podem ser desacopladas, obtendo:

$$
\left[m_{n}^{2} e^{2 k y}+e^{k y}\left(\partial_{5} e^{-k y} \partial_{5}\right)-c(c \pm 1) k^{2}\right] h_{L, R}^{(n)}=0,
$$

com o sinal "+" para o campo $L$ e " - " para o $R$. Analogamente ao caso do campo de gauge, a solução geral é dada por [35]:

$$
h_{L, R}^{(n)}(y)=\frac{e^{\frac{1}{2} k y}}{N_{n}}\left[J_{\left|c \pm \frac{1}{2}\right|}\left(\frac{m_{n} e^{k y}}{k}\right)+\beta_{\left|c \pm \frac{1}{2}\right|}\left(m_{n}\right) Y_{\left|c \pm \frac{1}{2}\right|}\left(\frac{m_{n} e^{k y}}{k}\right)\right]
$$

Podemos determinar as constantes $\beta_{\left|c \pm \frac{1}{2}\right|}\left(m_{n}\right)$ e $N_{n}$ pelas condições de contorno e pela normalização (2.49) das funções de onda, respectivamente. Se supormos que $\Psi_{L}$ é par sob $\mathbb{Z}_{2}$, obtemos de (2.51) a equação para o modo zero:

$$
\left(\partial_{5}+c k\right) h_{L}^{0}=0
$$

com a solução:

$$
h_{L}^{0}=A e^{-c k y}
$$

Essa solução obedece automaticamente a condição de contorno de Neumann modificada, que é identica à equação de movimento. Substituindo em (2.49), obtemos a solução normalizada:

$$
h_{L}^{(0)}(y)=\sqrt{\frac{k \pi R(1-2 c)}{e^{k \pi R(1-2 c)}-1}} e^{-c k y}
$$

Analogamente, se tivessemos escolhido $\Psi_{R}$ par, obteríamos o modo zero de mão 
direita:

$$
h_{R}^{(0)}(y)=\sqrt{\frac{k \pi R(1+2 c)}{e^{k \pi R(1+2 c)}-1}} e^{c k y} .
$$

Vemos que o parâmetro $c$ controla a localização do férmion na dimensão extra. Como o Higgs está localizado em $y=0$, o acoplamento de Yukawa (ver a seção (2.4) será grande para férmions localizados no IR e pequeno para férmions no UV. Dessa forma, com a escolha de parâmetros de ordem um, somos capazes de reproduzir a hierarquia de massas dos férmions. Para obter a dependência correta da localização com o parâmetro $c$, consideramos o termo cinético dos modos zero:

$$
S_{5}^{f}=\int d^{4} x \int_{0}^{\pi R} d y\left\{e^{k y} e^{ \pm 2 c k y}\left|A_{L, R}\right|^{2} \bar{\psi}_{L, R}^{(0)} i \gamma^{\mu} \partial_{\mu} \psi_{L, R}^{(0)}+\cdots\right\}
$$

com o sinal " - " para o campo $L$ e "+" para o $R$. Vemos que o fator que controla a localização é a exponencial:

$$
\begin{aligned}
& e^{\left(1 / 2-c_{L}\right) k y} \text { para mão esquerda, } \\
& e^{\left(1 / 2+c_{R}\right) k y} \text { para mão direita. }
\end{aligned}
$$

Assim, para $c_{L}>\frac{1}{2}$, o modo zero estará localizado próximo da brana UV, e para $c_{L}<\frac{1}{2}$ próximo da brana IR. O caso $c_{L}=\frac{1}{2}$ gera um modo zero plano. Analogamente, temos a localização próxima do UV (IR) para $c_{R}<-\frac{1}{2}\left(>-\frac{1}{2}\right)$ para o modo zero de mão direita. Comportamentos típicos para o caso de mão esquerda estão representados na figura (2.2).

Passaremos agora a estudar os acoplamentos de gauge e a violação de sabor no modelo de RS. 


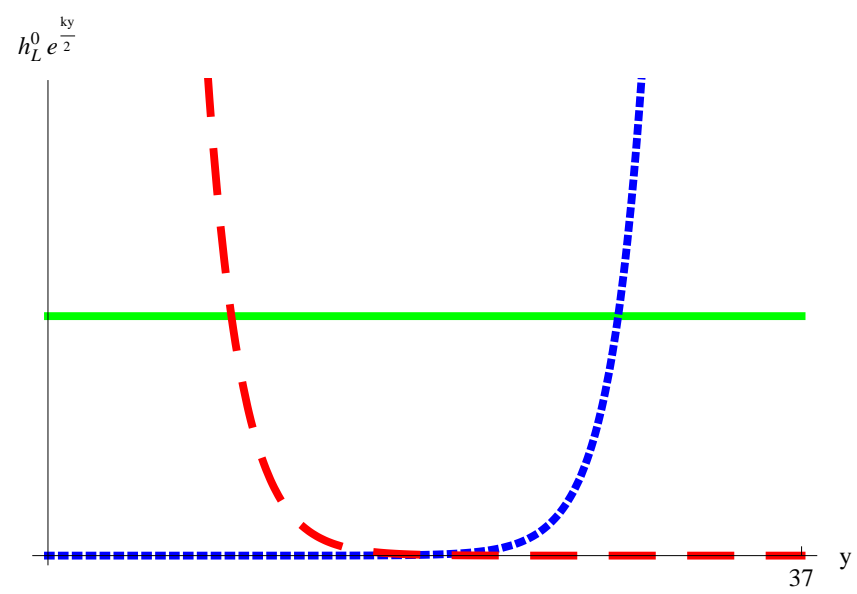

Figura 2.2: Função de onda do modo zero de mão esquerda em função da posição na dimensão extra, $y$. Os casos apresentados são $c_{L}=0.5$ (verde), $c_{L}=1.1$ (vermelho tracejado), $c_{L}=-0.1$ (azul pontilhado). A normalização do caso plano foi ajustada para permitir melhor visualização.

\subsection{Acoplamentos e Violação de Sabor}

Introduzindo interações de gauge para um férmion através da derivada covariante $D_{M}=\nabla_{M}-i g_{5} A_{M}$, geramos na ação cinco dimensional o termo:

$$
S_{\Psi A}^{5}=\int d^{4} x \int_{0}^{\pi R} d y \sqrt{g} g_{5} \bar{\Psi}\left(x^{\mu}, y\right) \Gamma^{\mu} A_{\mu}\left(x^{\mu}, y\right) \Psi\left(x^{\mu}, y\right)
$$

onde $g_{5}$ é o acoplamento de gauge 5-dimensional e trabalhamos no calibre $A_{5}=0$.

Em geral, interações que são renormalizáveis em quatro dimensões deixam de sê-lo em teorias com dimensões extras. De fato, uma vez que as dimensões canônicas de massa dos campos são $[\Psi]=2$ e $[A]=3 / 2$, a constante de acoplamento $g_{5}$ possui dimensão canônica $\left[g_{5}\right]=-1 / 2$, e portanto a interação de gauge é não renormalizável. Por essa razão, teorias cinco dimensionais devem ser vistas como teorias efetivas, com um corte $\Lambda$ acima do qual a teoria é fortemente acoplada e o espalhamento de modos de KK longitudinais (análogos a bósons de Goldstone) 
viola a unitariedade perturbativa [30]. Em teorias com dimensões extras curvas, a simetria de escala da métrica $A d S_{5}$ (equação (2.4)) faz com que a escala de física fortemente acoplada varie com a posição na dimensão extra segundo o fator de warping $e^{-k y}$. Se o corte na brana $U V$ for da ordem da escala de Planck, o correspondente corte na brana $I R$ será de ordem $\Lambda_{I R}=e^{-k \pi R} \Lambda_{U V} \simeq 1 \mathrm{TeV}$, que é a escala de massas do primeiro modo de KK [45].

Uma vez que a teoria é não renormalizável, devemos esperar a presença de operadores de dimensão superior como por exemplo, a interação de quatro férmions $\sim c_{i j k l} / \Lambda^{2} \bar{\psi}_{i} \psi_{j} \bar{\psi}_{k} \psi_{l}$. No entanto, em geral tais operadores serão suprimidos por um corte efetivo $\Lambda \sim M_{P}$ se ao menos um dos férmions estiver localizado no UV, o que garante a proteção contra efeitos fenomenologicamente indesejáveis, como o decaimento do próton (de fato essa é uma motivação para permitir a propagação dos férmions no bulk. Ver, por exemplo, [46]).

Vamos expandir os campos em modos de KK, usando (2.13) e (2.48), obtendo:

$$
\begin{array}{r}
S_{\Psi A}^{5}=\sum_{n, m, p} \frac{1}{(\pi R)^{3 / 2}} \int d^{4} x \int_{0}^{\pi R} d y g_{5}\left[h_{L, R}^{*(n)}(y) \bar{\psi}_{L, R}^{(n)}\left(x^{\mu}\right)\right] i \gamma^{\mu}\left[f^{(m)}(y) A_{\mu}^{(m)}\left(x^{\mu}\right)\right] \times \\
\\
{\left[h_{L, R}^{(p)}(y) \psi_{L, R}^{(p)}\left(x^{\mu}\right)\right] .}
\end{array}
$$

Podemos definir o acoplamento efetivo entre o n-ésimo modo de KK do férmion e o m-ésimo modo do campo de gauge escrevendo:

$$
S_{\Psi A}^{5}=g_{(n, m)}^{L, R} \int d^{4} x \bar{\psi}_{L, R}^{(n)}\left(x^{\mu}\right) i \gamma^{\mu} A_{\mu}^{(m)}\left(x^{\mu}\right) \psi_{L, R}^{(n)}\left(x^{\mu}\right)
$$


onde definimos:

$$
g_{(n, m)}^{L, R}=\frac{g_{5}}{(\pi R)^{3 / 2}} \int_{0}^{\pi R} d y e^{k y} f^{(m)}(y)\left|h_{L, R}^{(n)}(y)\right|^{2} .
$$

Para $m=n=0$, devemos recuperar o acoplamento de gauge usual do MP, $g$. Assim, utilizando $f^{(0)}(y)=1$ e a normalização das funções de onda dos férmions (2.49):

$$
g_{(0,0)} \equiv g=\frac{g_{5}}{\sqrt{\pi R}}
$$

Vemos que o acoplamento de gauge quadridimensional é suprimido por um fator que depende do tamanho da dimensão extra, $\sqrt{\pi R}$ em relação ao acoplamento em cinco dimensões. Essa é uma característica genérica de teorias com dimensões extras. Como desejamos que os modos zero dos férmions sejam identificados com os férmions do MP, estamos interessados nas modificações em sua física devido ao acoplamento com os modos de KK dos bósons de gauge. O acoplamento de maior relevância para isso é com o primeiro modo, $m=1$, já que sua massa é menor e portanto as interações mediadas pela troca deste modo estarão menos suprimidas. Reescrevendo o acoplamento em termos de $g$, ele será dado por:

$$
g_{(0,1)}^{L, R}=\frac{g}{(\pi R)} \int_{0}^{\pi R} d y e^{k y} f^{(1)}(y)\left|h_{L, R}^{(0)}(y)\right|^{2}
$$

A figura (2.3) mostra o comportamento desse acoplamento em unidades de $g$ quadridimensional como função da localização, para férmions de ambas as quiralidades.

Para $c_{L}<1 / 2$ ou $c_{R}>-1 / 2$ (localização no IR), a constante de acoplamento se aproxima assintoticamente do valor $g_{01}^{R} / g \simeq 8,4$, enquanto que para $c_{L}>1 / 2$ ou 


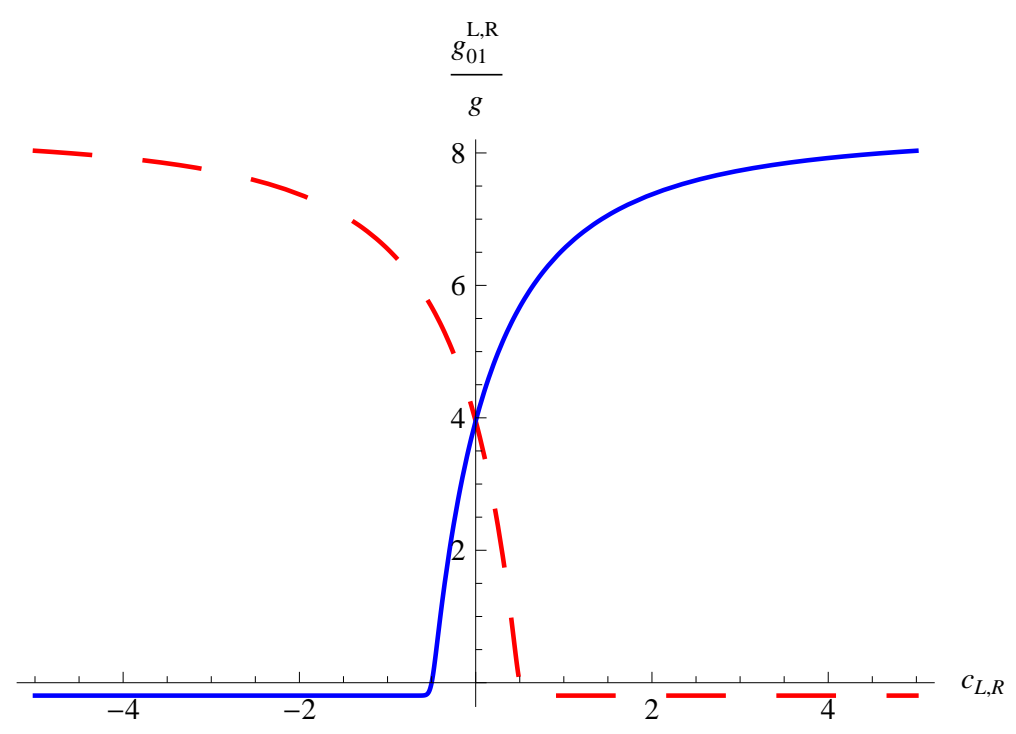

Figura 2.3: Acoplamento de gauge dos modos zero com o primeiro modo de KK de um bóson de gauge, em unidades do acoplamento do MP. Em vermelho tracejado (azul) temos o acoplamento de mão esquerda (direita) em função da localização $c_{L(R)}$.

$c_{R}<-1 / 2$ (localização no UV), ocorre saturação rápida, no valor $g_{01}^{R} / g \simeq-0,2$, [35]. Essa saturação pode ser entendida do fato de a função de onda do primeiro modo de KK do bóson de gauge ser pequena e aproximadamente constante no UV.

Os acoplamentos de Yukawa, por sua vez, são definidos por:

$$
\mathcal{L}_{\mathrm{Y}}^{5}=\sum_{i, j=1}^{3}\left[\int d^{4} x \int_{0}^{\pi R} d y \sqrt{g} \mathrm{Y}_{i j}^{5} \bar{\Psi}_{i}\left(x^{\mu}, y\right) \delta(y-\pi R) H\left(x^{\mu}\right) \Psi_{j}\left(x^{\mu}, y\right)\right]
$$

onde $i, j=1,2,3$ são índices de geração e $H\left(x^{\mu}\right)$ é o dubleto de Higgs do MP. Como no caso do acoplamento de gauge, a interação é não-renormalizável, e os acoplamentos de Yukawa $Y_{i j}^{5}$ terão dimensão canônica de massa $\left[Y_{i j}^{5}\right]=-1$. Assim, podemos escrever $\mathrm{Y}_{i j}^{5} \equiv \lambda_{i j}^{5} / k \operatorname{com} \lambda_{i j}^{5}$ adimensional e $k$ a curvatura de $A d S_{5}$. 
Vamos assumir que $\left|\lambda_{i j}^{5}\right| \sim \mathcal{O}(1)$, de maneira que a hierarquia de massas não será gerada por esses parâmetros. Novamente, tomamos o modelo mais simples em que a localização do Higgs é dada por uma função $\delta(y-\pi R)$. O acoplamento do modo zero dos férmions com o Higgs pode ser obtido utilizando a decomposição em modos de KK:

$$
\Psi_{L, R} \equiv \frac{e^{2 k y}}{\sqrt{\pi R}} \sum_{n=0}^{\infty} h_{L, R}^{(n)}(y) \psi_{L, R}^{(n)}\left(x^{\mu}\right),
$$

$\operatorname{com} n=0$. Fazendo isso, a lagrangiana (2.65) pode ser escrita como

$$
\begin{aligned}
& \mathcal{L}_{\mathrm{Y}}^{5}=\sum_{i, j=1}^{3} \frac{\mathrm{Y}_{i j}^{5}}{\pi R} \int d^{4} x \int_{0}^{\pi R} d y \delta(y-\pi R)\left\{\left[h_{L}^{(0)}(y) \bar{\psi}_{L}^{(0)}\left(x^{\mu}\right)\right]_{i} H\left(x^{\mu}\right) \times\right. \\
& {\left.\left[h_{R}^{(0)}(y) \psi_{R}^{(0)}\left(x^{\mu}\right)\right]_{j}+\text { h.c. }\right\} . }
\end{aligned}
$$

Devemos escrever todos os acoplamentos de Yukawa permitidos pelas simetrias de gauge. Em particular, se definirmos os campos dos quarks como:

$$
\begin{aligned}
Q_{L} & =\left\{\left(\begin{array}{l}
u \\
d
\end{array}\right)_{L},\left(\begin{array}{c}
c \\
s
\end{array}\right)_{L},\left(\begin{array}{l}
t \\
b
\end{array}\right)_{L}\right\}, \\
U_{R} & =\left\{u_{R}, c_{R}, t_{R}\right\} \\
D_{R} & =\left\{d_{R}, s_{R}, b_{R}\right\}
\end{aligned}
$$

e efetuarmos a integral (trivial) em $y$, podemos escrever as interações de Yukawa dos quarks no modelo de RS como:

$$
\mathcal{L}_{\mathrm{Y}}^{4}=\sum_{i, j=1}\left\{\int d^{4} x \mathrm{Y}_{i, j}^{U} \bar{Q}_{L i}^{(0)} H U_{R j}^{(0)}+\mathrm{Y}_{i, j}^{D} \bar{D}_{R j}^{(0)} \tilde{H} Q_{L i}^{(0)}+\text { h.c. }\right\},
$$

onde omitimos a dependência em $x^{\mu}$ dos campos quadridimensionais e definimos o dubleto conjugado de carga $\tilde{H} \equiv-i \sigma_{2} H^{*}$, com a matriz de Pauli $\sigma_{2}$. Defi- 
nimos as matrizes:

$$
\begin{aligned}
Y_{i j}^{U} & \equiv \frac{\lambda_{i j}^{5(U)}}{\pi R k} e^{k \pi R} h_{L i}^{(0) Q}(\pi R) h_{R j}^{(0) U}(\pi R), \\
Y_{i j}^{D} & \equiv \frac{\lambda_{i j}^{5(D)}}{\pi R k} e^{k \pi R} h_{L i}^{(0) Q}(\pi R) h_{R j}^{(0) D}(\pi R) .
\end{aligned}
$$

Esses são os acoplamentos de Yukawa que devem corresponder aos do MP. Os fatores adicionais de $e^{k \pi R}$ surgem pois após integrar sobre a dimensão extra, devemos efetuar a redefinição (2.8) para obter o termo cinético canônico para o Higgs. Se multiplicarmos os acoplamentos de Yukawa pelo valor esperado de $H, v / \sqrt{2}$, obteremos as correspondentes matrizes de massas, $M_{i j}^{U}$ e $M_{i j}^{D}$. Ao substituirmos as expressões para as funções de onda do modo zero (equações (2.55) e (2.56)), vemos que o acoplamento de Yukawa terá a forma funcional: ${ }^{8}$

$$
Y_{i j} \equiv \lambda_{i j}^{5} \sqrt{\frac{\left(1-2 c_{L}\right)}{e^{k \pi R\left(1-2 c_{L}\right)}-1}} \sqrt{\frac{\left(1+2 c_{R}\right)}{e^{k \pi R\left(1+2 c_{R}\right)}-1}} e^{k \pi R\left(1-c_{L}+c_{R}\right)} .
$$

Embora os parâmetros $\lambda_{i j}^{5}$ e $c_{L, R}$ sejam não hierárquicos, as matrizes de Yukawa obtidas do modelo de WED são capazes de gerar a hierarquia de massas dos férmions, pois as funções de onda dos modos zero fermiônicos, $h_{L, R}^{(0)}$ dependem exponencialmente da localização [35]. Como casos típicos, tomando o limite:

$$
\begin{aligned}
& e^{\left(1-2 c_{L}\right) k \pi R} \gg 1, \\
& e^{\left(1+2 c_{R}\right) k \pi R} \gg 1,
\end{aligned}
$$

\footnotetext{
${ }^{8} \mathrm{Na}$ realidade, a presença do Higgs na brana IR modifica as condições de contorno das equações de movimento. Assim existe uma mistura entre os modos zero e os modos de $K K$, e as expressões (2.55) e (2.56) possuem correções de ordem $O\left(m_{f}^{2} / M_{K K}^{2}\right)$ [47, 48], que ignoramos aqui.
} 
temos o acoplamento de Yukawa de um férmion fortemente localizado no IR:

$$
Y_{i j}^{I R} \equiv \lambda_{i j}^{5} \sqrt{\left(1-2 c_{L}\right)\left(1+2 c_{R}\right)} .
$$

Esse acoplamento é de ordem $\mathcal{O}(1)$, portanto, para férmions localizados no $I R$, como por exemplo o top, devemos obter massas de ordem $v$. Já para a localização no $U V$, tomamos o limite oposto:

$$
\begin{aligned}
& e^{\left(1-2 c_{L}\right) k \pi R} \ll 1, \\
& e^{\left(1+2 c_{R}\right) k \pi R} \ll 1 .
\end{aligned}
$$

Obtemos o acoplamento da forma:

$$
Y_{i j}^{U V} \equiv \lambda_{i j}^{5} \sqrt{\left(2 c_{L}-1\right)\left(2 c_{R}-1\right)} e^{k \pi R\left(1-c_{L}+c_{R}\right)},
$$

que é exponencialmente suprimido, permitindo obter massas pequenas para férmions localizados no $U V$, como o quark up.

A resolução do problema da hierarquia de massas dos férmions tem como preço a geração de correntes neutras que trocam sabor (flavor changing neutral currents - FCNC) a nível árvore. De fato, lembramos que no MP as correntes neutras para os quarks de mão esquerda e carga positiva tem a forma:

$$
\mathcal{L}_{\text {quarks }}^{(0)}=-\frac{g}{\cos \theta_{W}}\left(g_{u_{L}} \bar{u}_{L} \gamma^{\mu} u_{L}+g_{c_{L}} \bar{c}_{L} \gamma^{\mu} c_{L}+g_{t_{L}} \bar{t}_{L} \gamma^{\mu} t_{L}\right) Z_{\mu}
$$

Os acoplamentos são universais: $g_{u_{L}}=g_{c_{L}}=g_{t_{L}}=\left[1 / 2-(2 / 3) \sin ^{2} \theta_{W}\right] \equiv g_{L}^{(u p)}$, o que torna a corrente neutra invariante por uma rotação unitária $L_{U}$ para auto- 
estados de massa:

$$
\begin{gathered}
\mathcal{L}_{\text {quarks }}^{(0)}=-\frac{g}{\cos \theta_{W}}\left(\begin{array}{ccc}
\bar{u}^{\prime} & \bar{c}^{\prime} & \bar{t}^{\prime}
\end{array}\right)_{L} L_{U}^{-1}\left(\begin{array}{ccc}
g_{L} & 0 & 0 \\
0 & g_{L} & 0 \\
0 & 0 & g_{L}
\end{array}\right) L_{U} \gamma^{\mu}\left(\begin{array}{c}
u^{\prime} \\
c^{\prime} \\
t^{\prime}
\end{array}\right)_{L} Z_{\mu} \Rightarrow \\
\mathcal{L}_{\text {quarks }}^{(0)}=-\frac{g}{\cos \theta_{W}} g_{L}\left(\bar{u}_{L}^{\prime} \gamma^{\mu} u_{L}^{\prime}+\bar{c}_{L}^{\prime} \gamma^{\mu} c_{L}^{\prime}+\bar{t}_{L}^{\prime} \gamma^{\mu} t_{L}^{\prime}\right) Z_{\mu} .
\end{gathered}
$$

O mesmo ocorre para os outros quarks, caracterizando o chamado mecanismo GIM [49], que proíbe as FCNC a nível árvore. Esses efeitos que só aparecem no MP a ordem de 1-loop, através de diagramas de caixa. Por exemplo, a mistura entre os mésons $K_{0}$ e $\bar{K}_{0}$ é dada pelos diagramas da figura (2.4).
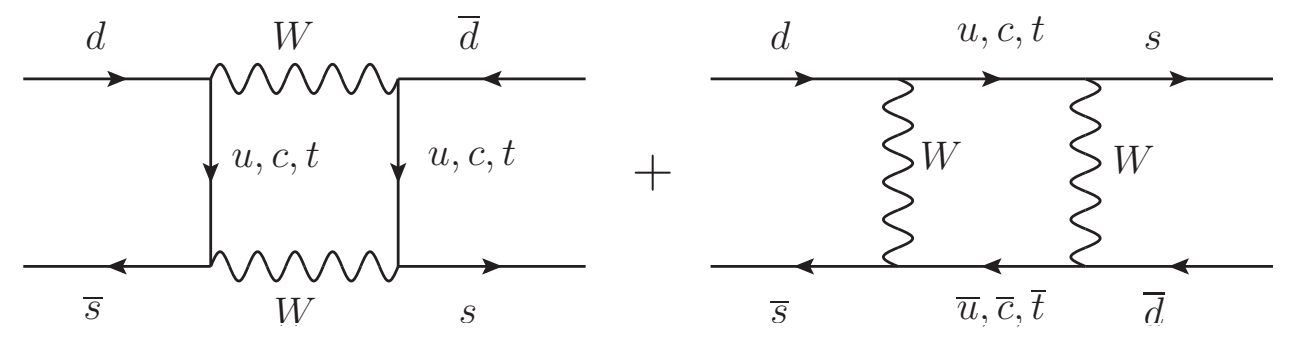

Figura 2.4: Diagramas de caixa que contribuem para a violação de sabor no MP a 1-loop, no setor de Kaons.

No entanto, a universalidade dos acoplamentos que garante o mecanismo GIM é violada se tentarmos gerar a hierarquia de massas dos férmions em um modelo de $W E D$, pois necessitamos de localizações diferentes para cada sabor. De fato, vemos da figura (2.3) que o acoplamento correspondente entre os quarks de mão esquerda do MP e o primeiro modo de KK do glúon, por exemplo, não será em geral universal, mas sim caracterizado por uma matriz no espaço de sa- 
bor:

$$
G_{U}^{L} \equiv L_{U}^{-1}\left(\begin{array}{ccc}
g_{(0,1)}^{L(1)} & 0 & 0 \\
0 & g_{(0,1)}^{L(2)} & 0 \\
0 & 0 & g_{(0,1)}^{L(3)}
\end{array}\right) L_{U},
$$

onde o índice $(i)=1,2,3$ representa a geração. Analogamente, definimos os correspondentes matrizes de acoplamento para os quarks tipo down de mão esquerda e os quarks de mão direita:

$$
\begin{aligned}
G_{D}^{L} & \equiv L_{D}^{\dagger} g_{(0,1)}^{L} L_{D}, \\
G_{U}^{R} & \equiv R_{U}^{\dagger} g_{(0,1)}^{R(U)} R_{U}, \\
G_{D}^{R} & \equiv R_{D}^{\dagger} g_{(0,1)}^{R(D)} R_{D},
\end{aligned}
$$

onde omitimos os índices de sabor e definimos as correspondentes transformações para auto-estados de massa, $L_{U}, R_{U}$ e $R_{D}$.

Embora exista essa violação de sabor a nível árvore, é possível mostrar que a estrutura hierárquica dos acoplamentos de Yukawa suprime grande parte dos processos de FCNC. Por analogia, esse novo mecanismo é chamado RS-GIM [50, 51, 52, 53]. Descreveremos de maneira simples como ele atua, segundo [38]. Assumindo por simplicidade duas famílias, consideramos por exemplo a violação de sabor no setor down de mão esquerda:

$$
G_{D}^{L} \equiv L_{D}^{-1}\left(\begin{array}{cc}
g_{(0,1)}^{L(1)} & 0 \\
0 & g_{(0,1)}^{L(2)}
\end{array}\right) L_{D} .
$$


Como $L_{D}$ é unitária, seus elementos satisfazem:

$$
\begin{aligned}
\left|L_{D(11)}\right|^{2}+\left|L_{D(12)}\right|^{2} & =1, \\
\left|L_{D(22)}\right|^{2}+\left|L_{D(21)}\right|^{2} & =1, \\
\left|L_{D(12)}\right|^{2}-\left|L_{D(21)}\right|^{2} & =0, \\
\left|L_{D(11)}\right|^{2}-\left|L_{D(22)}\right|^{2} & =0, \\
L_{D(11)}^{*} L_{D(21)}+L_{D(12)}^{*} L_{D(22)} & =0, \\
L_{D(11)}^{*} L_{D(12)}+L_{D(21)}^{*} L_{D(22)} & =0 .
\end{aligned}
$$

O que permite escrever:

$$
G_{D}^{L} \equiv\left(\begin{array}{cc}
g_{(0,1)}^{L(1)} & \left(g_{(0,1)}^{L(1)}-g_{(0,1)}^{L(2)}\right) L_{D(11)} L_{D(21)}^{*} \\
\left(g_{(0,1)}^{L(1)}-g_{(0,1)}^{L(2)}\right) L_{D(11)}^{*} L_{D(21)} & g_{(0,1)}^{L(2)}
\end{array}\right)
$$

Vemos que os elementos fora da diagonal são proporcionais a diferença $g_{(0,1)}^{L(1)}$ $g_{(0,1)}^{L(2)}$. Assim, se esses acoplamentos forem similares, a violação de sabor será suprimida. De fato, para a primeira e segunda famílias, podemos localizar os dubletos no $U V$ e portanto estamos na região $c_{L}>1 / 2$ [35], e assim, como mostrado na figura (2.3), o acoplamento é universal. Para a terceira família, deveremos ter $c_{L}<1 / 2$, para localizar o quark top no IR, mas nesse caso os vínculos experimentais são mais fracos [54] e permitem $M_{K K}>2 \mathrm{TeV}$ [35] para o primeiro estado massivo do glúon. Dessa forma, através do mecanismo RS-GIM, a maior parte dos processos de violação de sabor é suficientemente suprimida e não gera vínculos muito restritivos para as massas dos modos de KK. No entanto, foi mostrado em [55] que a violação de CP no setor de káons $\left(\epsilon_{\text {káon }}\right)$ possui contribuiçõoes 
vindas de acoplamentos com modos de KK que são problemáticas. As contribuições para o coeficiente de operadores do tipo $\bar{d}_{R}^{i} s_{L}^{i} \bar{d}_{L}^{j} s_{R}^{j}$ são maiores do que os de interações da forma $\bar{d}_{L}^{i} \gamma^{\mu} s_{L}^{i} \bar{d}_{L}^{j} \gamma^{\mu} s_{L}^{j}$ por um fator de aproximadamente 140 [56]. Isso gera um vínculo para a massa dos modos de KK dado por $M_{K K}>30 \mathrm{TeV}$, que além de fenomenologicamente indesejável, reintroduziria uma hierarquia em relação a escala eletrofraca [55].

Através de novas simetrias globais no setor de sabor, é possível reduzir o vínculo a $M_{K K}>3 \mathrm{TeV}[57,56]$. No entanto, a necessidade de introduzir novas simetrias de maneira ad hoc para eliminar a violação de sabor nos motiva a buscar novos modelos que permitam reproduzir a hierarquia de massas sem gerar as FCNCs. Nesse espírito, introduziremos a técnica de Desconstrução Dimensional para obter modelos quadridimensionais inspirados no modelo de RS que permitem solucionar os problemas de hierarquia e que, ao mesmo tempo, suprimem naturalmente a violação de sabor a nível árvore. 


\section{Capítulo 3}

\section{Desconstrução Dimensional}

\subsection{Introdução}

Embora o modelo de RS seja capaz de resolver os problemas de hierarquia de gauge e das massas dos férmions, vimos que ele sofre de problemas com violação de sabor. Além disso, teorias de gauge em mais de quatro dimensões sempre possuem acoplamentos com dimensão dada por $[g]=M^{-d / 2}$ onde $d$ é o número de dimensões extras e portanto, são teorias efetivas com um corte equivalente a um número máximo de modos de KK que podemos incluir sem violar a unitariedade perturbativa.

A baixas energias, podemos construir uma teoria efetiva quadridimensional contendo interações renormalizáveis, e cujo espectro seja semelhante ao espectro de modos de KK, em um certo limite. No entanto, essa teoria não necessita ter o mesmo comportamento a altas energias de uma teoria com dimensões extras, e na verdade admite outros completamentos ultravioleta. Essa abordagem se chama Desconstrução Dimensional (concebida independentemente por [29] e 
[30]) e com ela esperamos obter modelos que preservem as boas caracteristicas das dimensões extras, como a resolução dos problemas de hierarquia, mas evitando alguns de seus problemas. Descreveremos a seguir como construir um modelo deste tipo, seguindo basicamente a notação e o tratamento empregados em [58] e [59].

Consideramos uma teoria de calibre em quatro dimensões com o grupo de gauge dado pelo produto:

$$
G=G_{0} \times G_{1} \times \ldots \times G_{N-1} \times G_{N}
$$

onde em geral temos diferentes grupos de gauge $G_{j}=S U(m)_{j}$ para cada sítio $j=0,1, \ldots, N$. Incluímos também um conjunto de campos escalares $\Phi_{j}$ que se transformam na representação fundamental de $G_{j-1}$ e antifundamental de $G_{j}$ :

$$
\Phi_{j} \rightarrow U_{j-1} \Phi_{j} U_{j}^{\dagger}
$$

Esses campos se chamam campos de ligação e definem a estrutura da teoria, convenientemente representada em diagramas chamados de moose ou quiver. $\mathrm{O}$ tipo mais simples de diagrama quiver que podemos construir é a cadeia linear da figura (3.1). Nesses diagramas, cada círculo representa um sítio com uma simetria de calibre $S U(m)_{j}$, e as linhas tracejadas representam os campos de ligação, com a convenção de que o campo de ligação pertence a representação fundamental do grupo de onde a seta está saindo e antifundamental do círculo onde está entrando.

No limite de $N$ muito grande, mostraremos que certas teorias desconstruídas correspondem a dimensões extras discretizadas, e assim, a energias muito meno- 


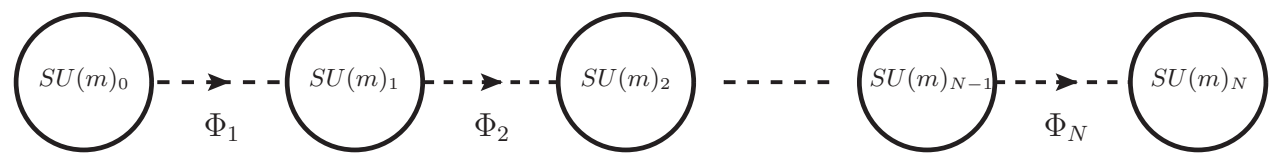

Figura 3.1: Diagrama quiver para uma cadeia linear de grupos de gauge.

res que o espaçamento da rede, ambas as teorias devem ser indistinguíveis. A estrutura geométrica da cadeia linear lembra um segmento de reta, e de fato essa configuração nos interessa pois desejamos que esse modelo se pareça com o modelo cinco-dimensional de Randall-Sundrum (que está compactificado em um orbifold $S^{1} / \mathbb{Z}_{2}$ ) no limite de baixas energias e grande número de sítios. No entanto, a estrutura dos campos de ligação é em principio arbitrária e pode representar qualquer tipo de variedade ou pode ainda não ter interpretação simples como dimensão extra.

Nas seções seguintes construiremos a ação desse tipo de modelo para o setor de gauge e de férmions, e iniciaremos o estudo do setor de Higgs. Estamos interessados em uma teoria com um número pequeno de sítios, que apresentará propriedades interessantes, bastante distintas da teoria extra-dimensional. Em particular, mostraremos que as violações de sabor são naturalmente suprimidas para poucos sítios. Em última análise, o modelo de RS será apenas uma inspiração e a teoria com poucos sítios deverá ser vista como um modelo puramente quadridimensional, sem interpretação como dimensão extra. 


\subsection{Bósons de Gauge}

A ação do setor de gauge da cadeia linear é escrita como:

$$
S_{4}^{A}=\int d^{4} x\left\{-\frac{1}{2} \sum_{j=0}^{N} \operatorname{Tr}\left[F_{\mu \nu, j} F_{j}^{\mu \nu}\right]+\sum_{j=1}^{N} \operatorname{Tr}\left[\left(\mathcal{D}_{\mu} \Phi_{j}\right)^{\dagger}\left(\mathcal{D}^{\mu} \Phi_{j}\right)\right]-V(\Phi)\right\}
$$

Os tensores de intensidade dos campos de gauge são $F_{\mu \nu, j}=F_{\mu \nu, j}^{a} T^{a}, a=$ $1,2, \ldots, m^{2}-1 \mathrm{com}$

$$
F_{\mu \nu, j}^{a}=\partial_{\mu} A_{\nu, j}^{a}-\partial_{\nu} A_{\mu, j}^{a}+g_{j} f_{j}^{a b c} A_{\mu, j}^{b} A_{\nu, j}^{c} .
$$

A derivada covariante é dada por:

$$
\mathcal{D}_{\mu} \Phi_{j}=\partial_{\mu} \Phi_{j}+i g_{j-1} A_{\mu, j-1}^{a} T_{j-1}^{a} \Phi_{j}-i g_{j} \Phi_{j} A_{\mu, j}^{a} T_{j}^{a}
$$

Identificamos a ação dessa teoria com uma soma de modelos sigma [60]. Se "desligarmos" os acoplamentos de gauge, a teoria exibirá a simetria global $S U(m)_{j-1} \times S U(m)_{j}$ para cada campo de ligação, análoga a simetria quiral da QCD. Se assumirmos que o potencial é tal que os campos adquirem um vev diagonal, podemos parametrizar, no limite não linear:

$$
\Phi_{j}=v_{j} e^{\left(i \pi_{j}^{a}(x) T_{j}^{a}\right) / v_{j}},
$$

onde $\pi_{j}^{a}$ são os bósons de Nambu-Goldstone da quebra da correspondente "simetria quiral" $S U(m)_{j-1} \times S U(m)_{j}$ ao subgrupo diagonal. A quebra de cada simetria ocorre na repectiva escala $v_{j}$. Escrevendo $v_{j} \equiv v q^{j}$, com $0<q<1$ temos uma 
sequência decrescente de vevs de 0 a $N .{ }^{1}$ Esta escolha permitirá efetuar a correspondência com o modelo de RS adiante. Como no caso da QCD, os campos de ligação são apenas uma descrição efetiva a baixas energias, e requerem completamentos ultravioleta, como por exemplo, condensados quirais de férmions [29].

A partir deste ponto, vamos tomar todos os grupos de gauge idênticos, ou seja, para todos os sítios, $T_{j}^{a} \equiv T^{a}$ e $g_{j} \equiv g$, o que corresponde a impor uma simetria de translação discreta $j \rightarrow j+1$. Antes de prosseguir, destacamos uma sutileza: a nível quântico, devemos especificar a escala de energia em que avaliamos as constantes de acoplamento. Para obter uma evolução do grupo de renormalização que é semelhante a do acoplamento de gauge na teoria com dimensões extras curvas, a escolha adequada é $[62,63]$ :

$$
g_{0}(v)=g_{1}\left(v_{1}\right)=\ldots=g_{N}\left(v_{N}\right)=g .
$$

Expandindo o termo cinético de $\Phi$, obtemos:

$$
\begin{aligned}
\operatorname{Tr}\left[\left(D_{\mu} \Phi_{j}\right)^{\dagger}\left(D^{\mu} \Phi_{j}\right)\right] & =\operatorname{Tr}\left[\left(\partial_{\mu} \Phi_{j}\right)^{\dagger}\left(\partial^{\mu} \Phi_{j}\right)\right]+g \operatorname{Tr}\left[i\left(\partial^{\mu} \Phi_{j}\right)^{\dagger}\left(A_{\mu, j-1} \Phi_{j}-\Phi_{j} A_{\mu, j}\right)+\text { h.c. }\right] \\
& +\frac{v_{j}^{2} g^{2}}{2}\left(A_{\mu, j}^{a 2}+A_{\mu, j-1}^{a 2}\right)-2 g^{2} \operatorname{Tr}\left[\Phi_{j}^{\dagger} A_{\mu, j-1} \Phi_{j} A_{j}^{\mu}\right]
\end{aligned}
$$

Podemos entender mais facilmente a mistura entre os bósons de NG e os campos de gauge expandindo o campo de ligação nos termos com o traço a ordem mais baixa em $\pi_{j} / v_{j}$ e usando a normalização $\operatorname{Tr}\left[T^{a} T^{b}\right]=\delta^{a b} / 2$. Assim, obtemos:

$$
\operatorname{Tr}\left[\left(D_{\mu} \Phi_{j}\right)^{\dagger}\left(D^{\mu} \Phi_{j}\right)\right]=\frac{1}{2}\left[\partial_{\mu} \pi_{j}^{a}-v_{j} g\left(A_{\mu, j}^{a}-A_{\mu, j-1}^{a}\right)\right]^{2} .
$$

\footnotetext{
${ }^{1}$ Embora não tenhamos feito a análise do potencial escalar dos campos de ligação, é possível obter os vevs com o perfil decrescente sem grande ajuste fino dos parâmetros do potencial [61].
} 
Essa mistura pode ser removida nos gauges $R_{\xi}$, introduzindo o termo de fixação de gauge: ${ }^{2}$

$$
\mathcal{L}_{G}=-\sum_{j=0}^{N} \frac{1}{2 \xi_{j}}\left[\partial_{\mu} A_{j}^{\mu, a}+\xi_{j} g\left(v_{j} \pi_{j}^{a}-v_{j+1} \pi_{j+1}^{a}\right)\right]^{2}
$$

É suficiente tomar $\xi_{j}=\xi$ para todos os sítios. É fácil ver que o termo cruzado cancela os termos de mistura após uma integração por partes. Assim, no gauge $R_{\xi}$, obtemos a Lagrangiana:

$$
\begin{aligned}
& S_{4}^{A}=\int d^{4} x\left\{-\frac{1}{2} \sum_{j=0}^{N} \operatorname{Tr}\left[F_{\mu \nu, j} F_{j}^{\mu \nu}\right]+\sum_{j=1}^{N} \frac{g^{2} v_{j}^{2}}{2}\left(A_{\mu, j}^{a}-A_{\mu, j-1}^{a}\right)^{2}+\right. \\
& \left.+\sum_{j=0}^{N} \frac{1}{2}\left(\partial_{\mu} \pi_{j}^{a}\right)\left(\partial^{\mu} \pi_{j}^{a}\right)-\sum_{j=1}^{N} \frac{g^{2} \xi}{2}\left(v_{j} \pi_{j}^{a}-v_{j+1} \pi_{j+1}^{a}\right)^{2}+\mathcal{O}\left(\frac{\pi_{j}^{3}}{v_{j}^{3}}\right)\right\} .
\end{aligned}
$$

Consideramos primeiro a Lagrangiana de massas dos bósons de NG:

$$
\mathcal{L}_{m_{\pi}}=-\sum_{j=1}^{N} \frac{g^{2} \xi}{2}\left(v_{j} \pi_{j}^{a}-v_{j+1} \pi_{j+1}^{a}\right)^{2} \equiv-\frac{1}{2} \pi^{a \dagger} M_{\pi}^{2} \pi^{a}
$$

onde $\pi^{a} \equiv\left\{\pi_{1}^{a}, \pi_{2}^{a}, \ldots, \pi_{N}^{a}\right\}^{t}$. A matriz de massas dos bósons de NG é dada por:

$$
M_{\pi}^{2}=g^{2} v^{2} \xi\left(\begin{array}{cccccc}
2 q^{2} & -q^{3} & 0 & \cdots & 0 & 0 \\
-q^{3} & 2 q^{4} & -q^{5} & \cdots & 0 & 0 \\
0 & -q^{5} & 2 q^{6} & \cdots & 0 & 0 \\
\vdots & \vdots & \vdots & \ddots & \vdots & \vdots \\
0 & 0 & 0 & \cdots & 2 q^{2(N-1)} & -q^{2 N-1} \\
0 & 0 & 0 & \cdots & -q^{2 N-1} & 2 q^{2 N}
\end{array}\right)
$$

\footnotetext{
${ }^{2} \mathrm{~A}$ introdução de $\mathcal{L}_{G}$ é acompanhada dos correspondentes fantasmas de Fadeev-Popov, que não serão necessários em nossos cálculos a nível árvore.
} 
Para nossos propósitos, é suficiente notar que $\operatorname{Det}\left[M_{\pi}^{2}\right]=\left(g^{2} v^{2} \xi\right)^{N}(N+1) q^{N(N+1)}$ $\neq 0$ e assim, nos gauges $R_{\xi}$ todos os bósons de NG obtém uma massa dependente do gauge (não física) proporcional a $\sqrt{\xi}$. O gauge unitário é obtido no limite $\xi \rightarrow \infty$, desacoplando completamente os bósons de NG do espectro, o que é consequência do fato de que todos os grupos de gauge são iguais, e assim cada subgrupo diagonal contém exatamente os $m^{2}-1$ graus de liberdade para serem absorvidos pelos respectivos $m^{2}-1$ bósons de gauge. ${ }^{3}$ Como temos $N$ campos de ligação para $N+1$ sítios, restará uma simetria de gauge $S U(m)$ não quebrada, correspondente ao modo zero. Daqui em diante trabalharemos no gauge unitário, por simplicidade.

Para estabelecer o espectro de modos de gauge massivos, consideramos a Lagrangiana de massas dos $A_{j}^{\mu}$ :

$$
\mathcal{L}_{m_{A}}=\frac{g^{2}}{2} \sum_{j=1}^{N}\left[v_{j}\left(A_{\mu, j}^{a}-A_{\mu, j-1}^{a}\right)\right]^{2} \equiv \frac{1}{2} A_{\mu}^{a \dagger} M_{A}^{2} A^{\mu, a}
$$

$\operatorname{com} A_{\mu}^{a} \equiv\left\{A_{\mu, 0}^{a}, A_{\mu, 1}^{a}, \ldots, A_{\mu, N}^{a}\right\}^{t}$. A matriz de massas dos bósons de gauge é dada então por:

$$
M_{A}^{2}=g^{2} v^{2}\left(\begin{array}{cccccc}
q^{2} & -q^{2} & 0 & \cdots & 0 & 0 \\
-q^{2} & q^{2}+q^{4} & -q^{4} & \cdots & 0 & 0 \\
0 & -q^{4} & q^{4}+q^{6} & \cdots & 0 & 0 \\
\vdots & \vdots & \vdots & \ddots & \vdots & \vdots \\
0 & 0 & 0 & \cdots & q^{2(N-1)}+q^{2 N} & -q^{2 N} \\
0 & 0 & 0 & \cdots & -q^{2 N} & q^{2 N}
\end{array}\right)
$$

\footnotetext{
${ }^{3}$ Poderíamos ter ido diretamente ao gauge unitário fazendo a transformação de gauge $U_{j}=$ $v_{j} e^{\left(-i \pi_{j}^{a}(x) T_{j}^{a}\right) / v_{j}}$, mas o gauge $R_{\xi}$ será útil quando generalizarmos a cadeia linear para o caso em que o Higgs é um pseudo bóson de NG, no capítulo (4).
} 
Podemos diagonalizar essa matriz através da transformação unitária:

$$
A_{\mu, j}=\sum_{n=0}^{N} f_{j, n} A_{\mu, n}^{\prime}
$$

onde os $A_{\mu, n}^{\prime}$ são os auto-estados de massa.

Podemos extrair das equações de movimento de $A_{j}^{\mu}$ a equação que deve ser satisfeita pelos $f_{j, n}$ para diagonalizar (3.15). Por simplicidade, trataremos o caso abeliano. O caso não abeliano possui interações entre diferentes modos massivos que vem das equações de movimento não lineares, mas o espectro e as autofunções são identicos ao caso $U(1)$ e podem ser encontrados em [30].

As equações de Euler-Lagrange

$$
\frac{\partial \mathcal{L}}{\partial A_{\nu, j}}-\partial_{\mu}\left(\frac{\partial \mathcal{L}}{\partial\left(\partial_{\mu} A_{\nu, j}\right)}\right)=0
$$

aplicadas a Lagrangiana da teoria $U(1)$ resultam em um conjunto de equações de Maxwell acopladas pelos termos de massa:

$$
\left(\partial^{2} A_{j}^{\nu}-\partial^{\nu} \partial_{\mu} A_{j}^{\mu}\right)+g^{2} v_{j}^{2}\left(A_{j}^{\nu}-A_{j-1}^{\nu}\right)+g^{2} v_{j+1}^{2}\left(A_{j}^{\nu}-A_{j+1}^{\nu}\right)=0 .
$$

Substituindo a expansão (3.16), obtemos:

$$
\left(\delta_{\mu}^{\nu} \partial^{2}-\partial^{\nu} \partial_{\mu}\right) f_{j, n} A_{n}^{\prime \mu}+g^{2} v^{2} q^{2 j}\left[\left(1+q^{2}\right) f_{j, n}-f_{j-1, n}-q^{2} f_{j+1, n}\right] A_{n}^{\prime \nu}=0 .
$$

Para que os $A_{n}^{\prime \mu}$ sejam autoestados de massa, eles tem de satisfazer:

$$
\left(\delta_{\mu}^{\nu} \partial^{2}-\partial^{\nu} \partial_{\mu}\right) A_{n}^{\prime \mu}+m_{n}^{2} A_{n}^{\prime \nu}=0
$$


Tomando o gradiente $\partial_{\nu}$ dessa equação, vemos que $\partial_{\nu} A_{n}^{\prime \nu}=0$ e assim ela é equivalente a equação de Proca:

$$
\partial^{2} A_{n}^{\prime \nu}+m_{n}^{2} A_{n}^{\prime \nu}=0
$$

Substituindo esse resultado em (3.19), obtemos:

$$
-m_{n}^{2} f_{j, n}+g^{2} v^{2} q^{2 j}\left[\left(1+q^{2}\right) f_{j, n}-f_{j-1, n}-q^{2} f_{j+1, n}\right]=0 .
$$

Dividindo por $g^{2} v^{2} q^{2 j+1}$ chegamos a equação:

$$
\left[q+q^{-1}-q^{-1}\left(x_{n} q^{-j}\right)^{2}\right] f_{j, n}-q f_{j+1, n}-q^{-1} f_{j-1, n}=0
$$

onde definimos $x_{n} \equiv m_{n} / g v$. O fato de existir uma simetria $S U(m)$ não quebrada ou, equivalentemente, de o determinante de (3.15) ser nulo implica que a equação acima sempre admite uma solução para $m_{0}=0$, o modo zero. A equação fica:

$$
\left(q+q^{-1}\right) f_{j, 0}-q f_{j+1,0}-q^{-1} f_{j-1,0}=0 .
$$

Podemos obter uma solução impondo condições contorno tipo "Neumann" na cadeia de sítios:

$$
\begin{aligned}
f_{0, n} & =f_{-1, n}, \\
f_{N+1, n} & =f_{N, n} .
\end{aligned}
$$


Essas condições para $n=0$ sobre (3.24) implicam que:

$$
f_{j+1,0}=f_{j, 0}, \forall j
$$

ou seja, o modo zero tem o mesmo peso em todos os sítios, o que é análogo ao fato do modo zero na teoria de dimensões extras ter um perfil plano (ver a equação (2.24)). Podemos determinar a constante $f_{j, 0} \equiv f_{0}$ através da condição de normalização:

$$
\sum_{j=0}^{N} f_{j, n}^{2}=1 .
$$

Para o modo zero:

$$
\begin{aligned}
& \sum_{j=0}^{N} f_{j, 0}^{2}=1 \Rightarrow \\
& f_{0}=\frac{1}{\sqrt{N+1}} .
\end{aligned}
$$

Surpreendentemente, também é possível obter uma solução analítica para os outros estados massivos. Para isso, definimos (comparar com (2.25)):

$$
\begin{aligned}
t[j] & \equiv x_{n} q^{-j}, \\
F^{(n)}(t[j]) & \equiv q^{j} f_{j, n} .
\end{aligned}
$$

Substituindo essas definições em (3.23) obtemos uma assim chamada equação de q-diferenças:

$$
\left(q+q^{-1}-q^{-1} t^{2}\right) F^{(n)}(t)-F^{(n)}\left(t q^{-1}\right)-F^{(n)}(t q)=0 .
$$

Esse tipo de equação é conhecida na literatura matemática por equação de 
Hahn-Exton [64, 65], e é um análogo discreto de uma equação de Bessel:

$$
q^{-\frac{\nu}{2}}\left(q^{\nu}+1-q t^{2}\right) J_{\nu}\left(t q^{1 / 2} ; q\right)-J_{\nu}(t q ; q)-J_{\nu}(t ; q)=0
$$

Suas soluções são conhecidas por funções de q-Bessel e de q-Neumann.

A função de q-Bessel , $J_{\nu}(t ; q)$, é dada pela série:

$$
J_{\nu}(t ; q)=t^{\nu} \frac{\left(q^{\nu+1} ; q\right)_{\infty}}{(q ; q)_{\infty}} \sum_{j=0}^{\infty} \frac{(-1)^{j} q^{j(j+1) / 2}}{\left(q^{\nu+1} ; q\right)_{j}(q ; q)_{j}} t^{2 j}
$$

onde $(p ; q)_{k}$, chamados de fatoriais q-deslocados ou símbolos de q-Pochhammer, são definidos por:

$$
(p ; q)_{k}=\left\{\begin{array}{ll}
1, & \text { se } k=0, \\
\prod_{n=0}^{k-1}\left(1-p q^{n}\right) & \text { se } k \geq 1
\end{array},\right.
$$

para $p \in \mathbb{C}, \quad k \in \mathbb{Z}_{+}=\{0,1,2, \ldots\}$ e $(p ; q)_{\infty} \equiv \lim _{k \rightarrow \infty}(p ; q)_{k}$. Por sua vez, a função de q-Neumann $Y_{\nu}(t ; q)$, é definida por:

$$
Y_{\nu}(t ; q)=\frac{\Gamma_{q}(\nu) \Gamma_{q}(1-\nu)}{\pi} q^{-\nu^{2} / 2}\left[\cos (\pi \nu) q^{\nu / 2} J_{\nu}(t ; q)-J_{-\nu}\left(t q^{-\nu / 2} ; q\right)\right]
$$

para índice $\nu$ não inteiro. $\mathrm{O}$ caso de índice inteiro é obtido no limite $\nu \rightarrow n \epsilon \mathbb{Z}$. A função $\Gamma_{q}(\nu)$ é definida por:

$$
\Gamma_{q}(\nu)=\frac{(q ; q)_{\infty}}{\left(q^{\nu} ; q\right)_{\infty}}(1-q)^{1-\nu}
$$

e satifaz o limite $\lim _{q \rightarrow 1^{-}} \Gamma_{q}(\nu)=\Gamma(\nu)$, onde $\Gamma(\nu)$ é a função gama de Euler. 
O limite do contínuo é dado por:

$$
\lim _{q \rightarrow 1^{-}} J_{\nu}\left(t(1-q) ; q^{2}\right)=J_{\nu}(t)
$$

em que recuperamos as funções usuais contínuas de Bessel e Neumann [66, 67].

A equação (3.30) corresponde ao caso $\nu=1$ da equação de Hahn-Exton e sua solução é [66, 67]:

$$
F^{(n)}(t)=A J_{1}\left(t ; q^{2}\right)+B Y_{1}\left(t ; q^{2}\right)
$$

onde $A$ e $B$ são constantes. Podemos obter os $f_{j, n}$ de (3.29):

$$
f_{j, n}=N_{n} q^{-j}\left[J_{1}\left(x_{n} q^{-j} ; q^{2}\right)+\beta\left(x_{n} ; q^{2}\right) Y_{1}\left(x_{n} q^{-j} ; q^{2}\right)\right],
$$

onde $N_{n}$ pode ser determinada pela normalização (3.27). Pode-se mostrar que a escolha da constante:

$$
\beta\left(x_{n} ; q^{2}\right)=-\frac{J_{0}\left(x_{n} ; q^{2}\right)}{Y_{0}\left(x_{n} ; q^{2}\right)},
$$

é tal que $f_{j, n}$ satisfaz a condição de contorno (3.25) em $j=0$ [66]. O espectro de massas pode então ser determinado pela condição de contorno em $j=N$ :

$$
J_{0}\left(x_{n} ; q^{2}\right) Y_{0}\left(q^{-(N+1)} x_{n} ; q^{2}\right)-Y_{0}\left(x_{n} ; q^{2}\right) J_{0}\left(q^{-(N+1)} ; q^{2}\right)=0 .
$$

O limite $q \rightarrow 1^{-}, N \rightarrow \infty$ e $q^{N}$ fixo corresponde ao contínuo, onde recuperamos as soluções da teoria extra-dimensional (2.27) e o espectro de massas de modos de KK. Nesse limite, o espectro é dado por (comparar com (2.33)) [66]:

$$
m_{n} \simeq\left(n-\frac{1}{4}\right) \pi g v(1-q) q^{N}, \quad n>0
$$


Longe do contínuo $q \ll 1$, o espectro é exponencial, de maneira que apenas o primeiro modo massivo é relevante para a fenomenologia.

A correspondência com o contínuo para $N$ grande pode ser estabelecida comparando as ações da teoria desconstruída e do modelo de RS cinco dimensional discretizado na dimensão extra, e então identificando os parâmetros de ambas.

Primeiramente, tomamos a ação dos campos de gauge no modelo de RS (equação (2.10)) com a redefinição $A_{M} \rightarrow A_{M} / g_{5}$ (note que, por consistência, ao reescalar o campo $A_{M}$ devemos mudar sua dimensão canônica para $\left[A_{M}\right]=1$ ):

$$
\begin{aligned}
S_{5}^{A} & =\int d^{4} x \int_{0}^{\pi R} d y \sqrt{g}\left\{-\frac{1}{2 g_{5}^{2}} \operatorname{Tr}\left[F_{M N}^{2}\right]\right\} \\
& =\int d^{4} x \int_{0}^{\pi R} d y\left\{-\frac{1}{2 g_{5}^{2}} \operatorname{Tr}\left[F_{\mu \nu} F^{\mu \nu}\right]+\frac{1}{2 g_{5}^{2}} e^{-2 k y}\left(\partial_{5} A_{\mu}^{a}-\partial_{\mu} A_{5}^{a}\right)^{2}\right\}
\end{aligned}
$$

Efetuamos a discretização pelas substituições: ${ }^{4}$

$$
\begin{aligned}
\int_{0}^{\pi R} d y & \rightarrow \sum_{j=0}^{N} a \\
\partial_{5} A_{\mu} & \rightarrow \frac{A_{\mu, j}-A_{\mu, j-1}}{a},
\end{aligned}
$$

onde $a$ é o espaçamento da rede. Obtemos assim:

$$
S_{5}^{A}=\frac{a}{g_{5}^{2}} \int d^{4} x\left\{-\frac{1}{2} \sum_{j=0}^{N} \operatorname{Tr}\left[F_{\mu \nu, j} F_{j}^{\mu \nu}\right]+\sum_{j=1}^{N} \frac{e^{-2 k a j}}{2}\left(\frac{A_{\mu, j}^{a}-A_{\mu, j-1}^{a}}{a}-\partial_{\mu} A_{5, j}^{a}\right)^{2}\right\} .
$$

O limite contínuo é obtido quando $a \rightarrow 0, N \rightarrow \infty, N a=L$ fixo. Devemos

\footnotetext{
${ }^{4}$ Sempre existem ambiguidades na discretização, por exemplo, poderíamos ter tomado $\partial_{5} A_{\mu} \rightarrow \frac{A_{\mu, j+1}-A_{\mu, j}}{a}$.
} 
comparar essa ação com a ação desconstruída:

$$
S_{4}^{A}=\frac{1}{g^{2}} \int d^{4} x\left[-\frac{1}{2} \sum_{j=0}^{N} \operatorname{Tr}\left[F_{\mu \nu, j} F_{j}^{\mu \nu}\right]+\sum_{j=1}^{N} \frac{v^{2} g^{2} q^{2 j}}{2}\left(A_{\mu, j}^{a}-A_{\mu, j-1}^{a}-\partial_{\mu} \frac{\pi_{j}^{a}}{v_{j}}\right)^{2}\right]
$$

onde reescalamos $A_{j}^{\mu} \rightarrow A_{j}^{\mu} / g$ por conveniência. Para que a ação $S_{4}^{A}$ tenda ao modelo de RS no contínuo, vemos que precisamos fazer as identificações: ${ }^{5}$

$$
\begin{aligned}
\frac{g_{5}^{2}}{a} & \leftrightarrow g^{2} \\
v & \leftrightarrow \frac{1}{\sqrt{a} g_{5}}=\frac{1}{a g}, \\
q & \leftrightarrow e^{-k a} .
\end{aligned}
$$

Comparando as ações, vemos que os bósons de NG $\pi_{j}$ correspondem portanto ao campo $A_{5}$, portanto o campo de ligação $\Phi_{j}=v_{j} e^{i \pi_{j} / v_{j}}$ é o análogo discreto da linha de Wilson $e^{i \int_{a j}^{a(j+1)} d y A_{5}}$ [66]. Com essas identificações, para $N$ grande e energias $E \ll 1 / a$, ambos os modelos devem ser indistinguíveis. Notamos que uma vez que $v=1 / a g$, as massas dos bósons de gauge $m \sim g v$ não dependem do valor do acoplamento $g$. Isso garante que, no contínuo, a torre de KK tenha massas com origem puramente geométrica. Por outro lado, isso requer campos de ligação independentes para cada grupo de gauge simples no quiver.

Se quisermos reproduzir a hierarquia de RS entre as escalas $M_{P}=10^{19} \mathrm{GeV}$ e a escala $M_{E W}=1 \mathrm{TeV}$ (2.5), precisamos que o sítio $N$ seja identificado com a

\footnotetext{
${ }^{5}$ Uma identificação alternativa, com $v_{j}=v$ igual para todos os sítios e $g_{j}$ e $a_{j}$ variáveis pode ser encontrada em [68].
} 
brana IR $(y=\pi R)$, e o sítio zero com a brana UV $(y=0)$. Numericamente, temos:

$$
\begin{aligned}
e^{-k \pi R} & \leftrightarrow e^{-k a N} \sim \frac{M_{E W}}{M_{P}}=10^{-16} \Rightarrow \\
q=e^{-k a} & \sim e^{-\frac{16 \ln 10}{N}} \simeq e^{-\frac{37}{N}} .
\end{aligned}
$$

Essa escolha permite reproduzir a solução do problema da hierarquia de gauge obtida no modelo de RS. Tipicamente o modo massivo mais leve terá massa de ordem $g v_{N}=g v q^{N} \sim 1 \mathrm{TeV}$, com $g v=1 / a \sim M_{P}=10^{19} \mathrm{GeV}$. O espaçamento da rede $1 /$ a pode então ser identificado com o corte ultravioleta da teoria cincodimensional, $\Lambda \sim M_{P}$. Passaremos agora a apresentar como se dá a inclusão dos férmions na desconstrução, e posteriormente, como obter a solução análoga a do modelo de RS para o problema da hierarquia de massas, sem gerar grandes FCNC.

\subsection{Férmions}

Vamos incluir os férmions na teoria desconstruída. A ação fermiônica no gauge unitário será dada por:

$$
\begin{array}{r}
S_{4}^{f}=\int d^{4} x\left\{\sum_{j=0}^{N}\left[\bar{\psi}_{L, j} i \not \partial \psi_{L, j}+\bar{\psi}_{R, j} i \not \partial \psi_{R, j}+\left(\mu_{j} \bar{\psi}_{L, j} \psi_{R, j}+\text { h.c. }\right)\right]+\right. \\
\left.+\sum_{j=1}^{N} \lambda v_{j}\left(\bar{\psi}_{R, j-1} \psi_{L, j}+\text { h.c. }\right)\right\},
\end{array}
$$

onde utilizamos a decomposição quiral usual $\psi_{L, R} \equiv \frac{1 \mp \gamma^{5}}{2} \psi$ e $v_{j}$ vem do valor 
esperado de $\Phi_{j}$, como anteriormente. Os acoplamentos de Yukawa $\lambda$ sempre são entre o férmion de mão direita do sítio $j$ com o férmion de mão esquerda do sítio $j+1$, o que define a chamada direção de salto (hopping direction) da teoria. Podemos representar tal situação no diagrama quiver, mostrado na figura (3.2).

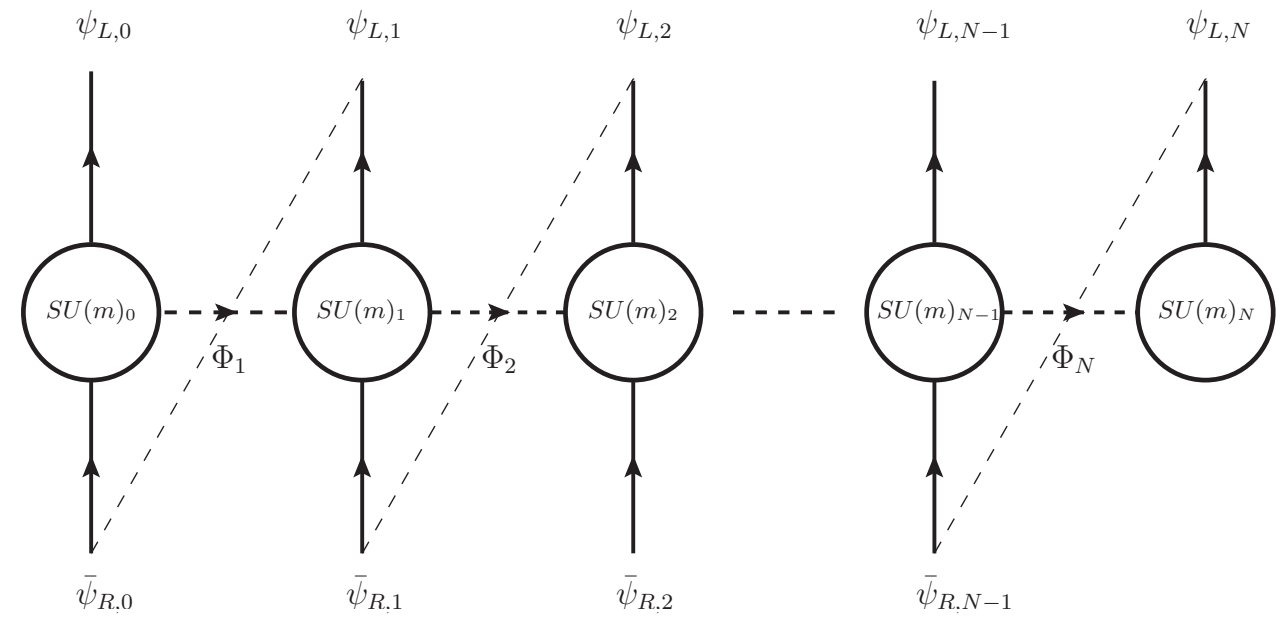

Figura 3.2: Diagrama quiver para uma cadeia linear de grupos de gauge incluindo os férmions.

As setas verticais representam os férmions. Novamente, a seta saindo indica a representação fundamental e a seta entrando a representação antifundamental com respeito ao grupo do círculo. A linha tracejada na diagonal indica os acoplamentos de Yukawa com os campos de ligação. Notamos a ausência do campo $\bar{\psi}_{R, N}$. Mais adiante veremos que essa é uma condição de contorno que impõe que o modo zero da teoria seja um campo de mão esquerda. Alternativamente, poderiamos ter $\psi_{L, 0}=0$, obtendo assim o modo zero de mão direita.

A Lagrangiana de massas dos férmions é:

$$
\mathcal{L}_{m f}=\sum_{j=0}^{N}\left(\mu_{j} \bar{\psi}_{L, j} \psi_{R, j}+\text { h.c. }\right)+\sum_{j=1}^{N} \lambda v_{j}\left(\bar{\psi}_{R, j-1} \psi_{L, j}+\text { h.c. }\right) \equiv \bar{\Psi}_{L} M_{f} \Psi_{R}+\text { h.c }
$$


onde $\Psi_{L} \equiv\left\{\psi_{L, 0}, \ldots, \psi_{L, N}\right\}^{t}$. Obtemos a matriz de massas para os férmions de mão esquerda fazendo:

$$
M_{f} M_{f}^{\dagger}=\left(\begin{array}{cccccc}
\mu_{0}^{2} & \lambda \mu_{0} v_{1} & 0 & \cdots & 0 & 0 \\
\lambda \mu_{0} v_{1} & \lambda^{2} v_{1}^{2}+\mu_{1}^{2} & \lambda \mu_{1} v_{2} & \cdots & 0 & 0 \\
0 & \lambda \mu_{1} v_{2} & \lambda^{2} v_{2}^{2}+\mu_{2}^{2} & \cdots & 0 & 0 \\
\vdots & \vdots & \vdots & \ddots & \vdots & \vdots \\
0 & 0 & 0 & \cdots & \lambda^{2} v_{N-1}^{2}+\mu_{N-1}^{2} & \lambda \mu_{N-1} v_{N} \\
0 & 0 & 0 & \cdots & \lambda \mu_{N-1} v_{N} & \lambda^{2} v_{N}^{2}
\end{array}\right) .
$$

Como no caso dos bósons de gauge, podemos diagonalizar essa matriz pela mudança de base:

$$
\psi_{L, j}=\sum_{n=0}^{N} h_{j, n}^{L} \psi_{L, n}^{\prime} .
$$

E analogamente, diagonalizamos $M_{f}^{T} M_{f}$ com a transformação:

$$
\psi_{R, j}=\sum_{n=0}^{N} h_{j, n}^{R} \psi_{R, n}^{\prime} .
$$

As equações de movimento podem ser obtidas de (3.48):

$$
\begin{array}{ll}
\text { Para } \bar{\psi}_{R, j}: & i \not \partial \psi_{R, j}+\lambda v_{j+1} \psi_{L, j+1}+\mu_{j} \psi_{L, j}=0, \\
\text { Para } \bar{\psi}_{L, j}: & i \not \partial \psi_{L, j}+\lambda v_{j} \psi_{R, j-1}+\mu_{j} \psi_{R, j}=0 .
\end{array}
$$

Os $\psi_{R, n}^{\prime}$ e $\psi_{L, n}^{\prime}$ terão massa bem definida se satisfizerem a equação de Dirac:

$$
\begin{aligned}
& i \not \partial \psi_{L, n}^{\prime}-m_{n} \psi_{R, n}^{\prime}=0, \\
& i \not \partial \psi_{R, n}^{\prime}-m_{n} \psi_{L, n}^{\prime}=0,
\end{aligned}
$$


obtemos de (3.53) e (3.54) que

$$
\begin{gathered}
m_{n} h_{j, n}^{R}+\mu_{j} h_{j, n}^{L}+\lambda v_{j+1} h_{j+1, n}^{L}=0 \\
m_{n} h_{j, n}^{L}+\mu_{j} h_{j, n}^{R}+\lambda v_{j} h_{j-1, n}^{R}=0 .
\end{gathered}
$$

É simples desacoplar essas equações:

$$
\begin{gathered}
\left(\mu_{j}^{2}+\lambda^{2} v_{j}^{2}-m_{n}^{2}\right) h_{j, n}^{L}+\lambda \mu_{j} v_{j+1} h_{j+1, n}^{L}+\lambda \mu_{j-1} v_{j} h_{j-1, n}^{L}=0, \\
\left(\mu_{j}^{2}+\lambda^{2} v_{j+1}^{2}-m_{n}^{2}\right) h_{j, n}^{R}+\lambda \mu_{j+1} v_{j+1} h_{j+1, n}^{R}+\lambda \mu_{j} v_{j} h_{j-1, n}^{R}=0 .
\end{gathered}
$$

Antes de prosseguir, gostaríamos de identificar os parâmetros livres $\mu_{j}$ e $\lambda$ com os correspondentes na Lagrangiana de RS, a fim de que ambas sejam equivalentes no limite do contínuo. Por conveniência, reproduzimos aqui a ação (2.46):

$$
\begin{array}{r}
S_{5}^{f}=\int d^{4} x \int_{0}^{\pi R} d y\left\{e^{k y} i \overline{\tilde{\Psi}}_{L, R} \gamma^{\mu} \partial_{\mu} \tilde{\Psi}_{L, R}-\right. \\
\left(\overline{\tilde{\Psi}}_{L} \partial_{5} \tilde{\Psi}_{R}-L \leftrightarrow R\right)+ \\
\left.+m_{\Psi}\left(\overline{\tilde{\Psi}}_{L} \tilde{\Psi}_{R}+\text { h.c. }\right)\right\} .
\end{array}
$$

Lembramos que $\tilde{\Psi}_{L, R}=e^{-2 k y} \Psi_{L, R}$. Antes de discretizar a ação, definimos os operadores de diferenças:

$$
\begin{aligned}
\nabla_{5} \Psi & \equiv \frac{\psi_{j+1}-\psi_{j}}{a} \\
\nabla_{5}^{*} \Psi & \equiv \frac{\psi_{j}-\psi_{j-1}}{a}
\end{aligned}
$$

onde a mudança $\Psi(x, y) \rightarrow \psi_{j}(x)$ é apenas uma notação para indicar que o campo 
$\psi_{j}$ é função apenas de $x^{\mu}$. Assim, tomando:

$$
\partial_{5} \Psi \rightarrow \frac{\nabla_{5}+\nabla_{5}^{*}}{2} \Psi=\frac{\psi_{j+1}-\psi_{j-1}}{2 a} .
$$

Como $\nabla_{5}^{\dagger}=-\nabla_{5}^{*}$, garantimos que a hermiticidade de $i \partial_{5}$ é preservada na discretização $[69,70,71] .^{6}$ Tomando o parâmetro de massa em unidades da curvatura $\mathrm{AdS}, m_{\Psi} \equiv c k$, sendo $c$ o parâmetro de localização do férmion, a ação discretizada é escrita:

$$
\begin{aligned}
S_{5}^{f}= & \int d^{4} x \sum_{j=0}^{N} a\left\{e^{k a j}\left(\bar{\psi}_{L, j} i \partial_{L} \psi_{L, j}+\bar{\psi}_{R, j} i \partial_{R} \psi_{R, j}\right)+c k\left(\bar{\psi}_{R, j} \psi_{L, j}+\text { h.c. }\right)\right. \\
& \left.+\frac{1}{2}\left(\bar{\psi}_{R, j} \psi_{L, j+1}-\bar{\psi}_{L, j} \psi_{R, j+1}+\text { h.c. }\right)\right\} .
\end{aligned}
$$

A ação discretizada sofre de um problema típico enfrentado em teorias de gauge na rede, chamado problema de duplicação dos férmions. Para ver isso, é útil considerar a ação no espaço de momentos. Usamos a representação integral da delta de Kronecker:

$$
\delta_{j, k}=\int_{-\pi / a}^{\pi / a} \frac{d p}{2 \pi} e^{i p(j-k) a}
$$

\footnotetext{
${ }^{6}$ Alternativamente, poderíamos ter tomado $\partial_{5} \rightarrow \nabla_{5}$, como foi feito para os bósons. Nesse caso, devemos adicionar divergências totais a ação cinco-dimensional para que ela seja hermitiana, como é feito em $[39,58]$.
} 
para escrever:

$$
\begin{aligned}
\int_{0}^{\pi R} d y \bar{\Psi} i \gamma^{5} \partial_{5} \Psi & \rightarrow \sum_{j, k=0}^{N} a \bar{\psi}_{j} i \gamma^{5} \frac{\left(\delta_{j, k+1}-\delta_{j, k-1}\right)}{2 a} \psi_{k} \\
& =\sum_{j, k=0}^{N} a \int_{-\pi / a}^{\pi / a} \frac{d p_{5}}{2 \pi} e^{i p_{5}(j-k) a} \bar{\psi}_{j} \gamma^{5} \frac{\operatorname{sen}\left(p_{5} a\right)}{a} \psi_{k}
\end{aligned}
$$

Vemos que o propagador fermiônico no espaço de momentos possui um polo adicional nos limites da zona de Brillouin, $p_{5}= \pm \pi / a$, dobrando o espectro fermiônico e, em particular, gerando dois modos zero (para $m \neq 0$ obtemos um polo para $p^{2} \sim m^{2}$ e um para $\left.p^{2} \sim(\pi / a)^{2}\right)$. Uma maneira de resolver esse problema é introduzindo o chamado termo de Wilson na ação $[69,70,71]:^{7}$

$$
\begin{aligned}
S_{\text {Wilson }} & =\eta a \int d^{4} x \int_{0}^{\pi R} d y \sqrt{g} \bar{\Psi}\left(\partial_{5}\right)^{2} \Psi \\
& \simeq \eta a \int d^{4} x \int_{0}^{\pi R} d y \overline{\tilde{\Psi}}\left(\partial_{5}\right)^{2} \tilde{\Psi}
\end{aligned}
$$

onde a igualdade da segunda linha é válida a menos de termos de ordem mais alta em $a$. Tal termo tende a zero no limite do contínuo $a \rightarrow 0$ (ou, na linguagem cinco-dimensional, é um operador irrelevante suprimido pelo corte $\Lambda=1 / a$ ), mas permitirá resolver o problema do espectro dobrado na teoria discretizada, através da escolha do parâmetro $\eta$. Para discretizar a ação de Wilson, definimos o Laplaciano discreto:

$$
\left(\partial_{5}\right)^{2} \Psi \rightarrow \nabla_{5}^{*} \nabla_{5} \Psi=\frac{\psi_{j+1}+\psi_{j-1}-2 \psi_{j}}{a^{2}}
$$

\footnotetext{
${ }^{7} \mathrm{~A}$ introdução de $S_{\text {Wilson }}$ em teorias quirais involve sutilezas com respeito a anomalias e simetria quiral. Nosso tratamento é incompleto, mas o leitor interessado pode encontrar mais detalhes em $[69,70,71,58]$.
} 
Assim, chegamos ao termo de Wilson discretizado:

$$
\begin{array}{r}
S_{\text {Wilson }}=\int d^{4} x \sum_{j=0}^{N} \eta\left\{\bar{\psi}_{R, j} \psi_{L, j+1}+\bar{\psi}_{L, j} \psi_{R, j+1}\right. \\
\left.-2 \bar{\psi}_{L, j} \psi_{R, j}+\text { h.c. }\right\} \text {. }
\end{array}
$$

Somamos então este termo a (3.64)

$$
\begin{aligned}
& S_{5}^{f}+S_{\text {Wilson }}= \\
& \int d^{4} x \sum_{j=0}^{N}\left\{\bar{\psi}_{L, j} i \partial_{L} \psi_{L, j}+\bar{\psi}_{R, j} i \not_{R} \psi_{R, j}+(c k a-2 \eta) \frac{e^{-k a j}}{a}\left(\bar{\psi}_{R, j} \psi_{L, j}+\text { h.c. }\right)\right. \\
& \left.+\frac{e^{-k a\left(j+\frac{1}{2}\right)}}{a}\left[\left(\eta-\frac{1}{2}\right) \bar{\psi}_{L, j} \psi_{R, j+1}+\left(\eta+\frac{1}{2}\right) \bar{\psi}_{R, j} \psi_{L, j+1}+\text { h.c. }\right]\right\}
\end{aligned}
$$

onde redefinimos $\psi_{j} \rightarrow \frac{e^{-\frac{k a j}{2}}}{\sqrt{a}} \psi_{j}$ para normalizar canonicamente o termo cinético. A escolha $\eta= \pm 1 / 2$ permite eliminar um dos dois modos zero. Assim, para obter o modo zero de mão esquerda, optamos por $\eta=1 / 2$, obtendo:

$$
\begin{aligned}
S_{L}^{f}= & \int d^{4} x \sum_{j=0}^{N}\left\{\bar{\psi}_{L, j} i \not_{L} \psi_{L, j}+\bar{\psi}_{R, j} i \not_{R} \psi_{R, j}\right. \\
& \left.-\frac{e^{-k a(j+c)}}{a}\left(\bar{\psi}_{R, j} \psi_{L, j}+\text { h.c. }\right)+\frac{e^{-k a\left(j+\frac{1}{2}\right)}}{a}\left(\bar{\psi}_{R, j} \psi_{L, j+1}+\text { h.c. }\right)\right\},
\end{aligned}
$$

onde notamos que $1-c k a=e^{-c k a}$ já que mantemos termos até $1^{\text {a }}$ ordem em $a$. Podemos agora comparar essa ação com a ação da teoria desconstruída (3.48). Impondo as condições de identificação obtidas para os bósons de gauge (3.46), 
vemos que devemos identificar os parâmetros livres como [58]:

$$
\mu_{j} \leftrightarrow-g v_{j} q^{c+1 / 2}, \quad \lambda \leftrightarrow g
$$

Notamos que a identificação do acoplamento de Yukawa $\lambda$ com o acoplamento de gauge $g$ é consequência do fato de que na teoria com dimensões extras, o campo de ligação está relacionado ao campo de gauge $A_{5}$. Do ponto de vista da teoria desconstruída, esse é um ajuste dos parâmetros que deve ser feito para obter o limite correto no contínuo.

Substituindo essas condições nas equações de movimento (3.59) e (3.60), chegamos a:

$$
\begin{aligned}
& {\left[q^{-\left(c+\frac{1}{2}\right)}+q^{\left(c+\frac{1}{2}\right)}-q^{-\left(c+\frac{1}{2}\right)}\left(x_{n} q^{-j+\frac{1}{2}}\right)^{2}\right] h_{j, n}^{L}-q h_{j+1, n}^{L}-q^{-1} h_{j-1, n}^{L}=0,} \\
& {\left[q^{-\left(c-\frac{1}{2}\right)}+q^{\left(c-\frac{1}{2}\right)}-q^{-\left(c-\frac{1}{2}\right)}\left(x_{n} q^{-j-\frac{1}{2}}\right)^{2}\right] h_{j, n}^{R}-q h_{j+1, n}^{R}-q^{-1} h_{j-1, n}^{R}=0,}
\end{aligned}
$$

onde $x_{n}=m_{n} / g v$. Novamente, podemos relacionar essas equações a equações de Hahn-Exton, e assim podemos escrever as correspondentes soluções em termos de funções de q-Bessel e q-Neumann:

$$
\begin{aligned}
& h_{j, n}^{L}=N_{n}^{L} q^{-j}\left[J_{\left|c+\frac{1}{2}\right|}\left(x_{n} q^{-j+\frac{1}{2}} ; q^{2}\right)+\beta_{\left|c+\frac{1}{2}\right|}\left(x_{n} ; q^{2}\right) Y_{\left|c+\frac{1}{2}\right|}\left(x_{n} q^{-j+\frac{1}{2}} ; q^{2}\right)\right], \\
& h_{j, n}^{R}=N_{n}^{R} q^{-j}\left[J_{\left|c-\frac{1}{2}\right|}\left(x_{n} q^{-j-\frac{1}{2}} ; q^{2}\right)+\beta_{\left|c-\frac{1}{2}\right|}\left(x_{n} ; q^{2}\right) Y_{\left|c-\frac{1}{2}\right|}\left(x_{n} q^{-j-\frac{1}{2}} ; q^{2}\right)\right]
\end{aligned}
$$

onde $N_{n}^{L}$ e $N_{n}^{R}$ são fatores de normalização e $\beta_{\left|c \pm \frac{1}{2}\right|}\left(x_{n} ; q^{2}\right)$ é determinado pela condição de contorno em $j=0$. O espectro é obtido da condição de contorno em $j=N$. 
Para analizar o modo zero, retornamos às equações (3.57) e (3.58). Para $m_{0}=$ 0, elas serão dadas por:

$$
\begin{gathered}
\mu_{j} h_{j, 0}^{L}+\lambda v_{j+1} h_{j+1,0}^{L}=0 \Rightarrow \\
\frac{h_{j+1,0}^{L}}{h_{j, 0}^{L}}=-\frac{\mu_{j}}{\lambda v_{j+1}}=q^{c_{L}-1 / 2}, \\
\mu_{j} h_{j, 0}^{R}+\lambda v_{j} h_{j-1,0}^{R}=0 \Rightarrow \\
\frac{h_{j-1,0}^{R}}{h_{j, 0}^{R}}=-\frac{\mu_{j}}{\lambda v_{j}}=q^{c_{R}+1 / 2} .
\end{gathered}
$$

A escolha das condições $\bar{\psi}_{R, N}=0$ ou $\psi_{L, 0}=0$ permite eliminar o modo zero de mão direita, ou esquerda, respectivamente. A escolha do parâmetro $c$ determinará qual dos sítios possui maior contribuição para o modo zero. No caso de mão esquerda, como $0<q<1$, , para $c_{L}>1 / 2$ os coeficientes $h_{j, 0}^{L}$ decrescem do sítio zero ao sítio $N$, e assim, analogamente ao caso contínuo (seção (2.3)), dizemos que o modo zero de mão-esquerda estará "localizado" próximo ao sítio zero, correspondente a brana $U V$, enquanto que para $c_{L}<1 / 2$ estará localizado próximo ao sítio $N$, correspondente a brana IR. Para o modo zero de mão direita, teremos para $c_{R}>-1 / 2$ a localização no sítio $N$, e para $c_{R}<-1 / 2$ a localização no sítio zero.

Definimos a normalização dos modos massivos dos férmions por:

$$
\sum_{j=0}^{N}\left|h_{j, n}^{L, R}\right|^{2}=1 .
$$


Para o modo zero, as relações (3.77) e (3.78) implicam:

$$
\frac{h_{1,0}^{L, R}}{h_{0,0}^{L, R}}=Z_{L, R},
$$

onde definimos $Z_{L} \equiv q^{c_{L}-1 / 2}$ e $Z_{R} \equiv q^{-\left(c_{R}+1 / 2\right)}$. Iterando sucessivas vezes, obtemos:

$$
h_{j, 0}^{L, R}=Z_{L, R}^{j} h_{0,0}^{L, R}
$$

Dessa forma, a normalização do modo zero é dada por:

$$
\sum_{j=0}^{N}\left|h_{j, 0}^{L, R}\right|^{2}=\left|h_{0,0}^{L, R}\right|^{2} \sum_{j=0}^{N} Z_{L, R}^{2 j}=1 .
$$

Efetuando a soma da série geométrica, concluímos que

$$
h_{0,0}^{L, R}=\left(\frac{1-Z_{L, R}^{2}}{1-Z_{L, R}^{2(N+1)}}\right)^{1 / 2}
$$

Passaremos agora a estudar os acoplamentos dos modos zero dos férmions aos bósons de gauge e ao Higgs, e extraíremos consequências da teoria desconstruída com poucos sítios para a violação de sabor.

\subsection{Acoplamentos}

Vamos considerar os acoplamentos de gauge na teoria desconstruída. Para um férmion de mão esquerda, eles serão dados por:

$$
\mathcal{L}_{g}=\sum_{j=0}^{N} \tilde{g} \bar{\psi}_{L, j} \gamma^{\mu} A_{\mu, j} \psi_{L, j},
$$


onde $\tilde{g}$ é o acoplamento de gauge, assumido universal para todos os sítios para que obtenhamos a teoria de RS no contínuo. Substituindo as expansões em modos massivos (3.16) e (3.51), obtemos:

$$
\mathcal{L}_{g}=\sum_{j, n, m, p=0}^{N}\left[\tilde{g}\left(h_{j, n}^{L}\right)^{*} f_{j, m} h_{j, p}^{L}\right] \bar{\psi}_{L, n}^{\prime} \gamma^{\mu} A_{\mu, m}^{\prime} \psi_{L, p}^{\prime} .
$$

Os acoplamentos na base de autoestados de massa podem então ser definidos por:

$$
g_{n, m, p}^{L} \equiv \tilde{g} \sum_{j=0}^{N}\left(h_{j, n}^{L}\right)^{*} f_{j, m} h_{j, p}^{L} .
$$

Estamos interessados no acoplamento com o modo zero do férmion, para estudar modificações na física do MP. Assim, fazemos $n=p=0$, obtendo:

$$
g_{0 m}^{L} \equiv \tilde{g} \sum_{j=0}^{N}\left|h_{j, 0}^{L}\right|^{2} f_{j, m} .
$$

Podemos obter a normalização correta do acoplamento fazendo $m=0$, e substituindo $f_{j, 0}=f_{0}=1 / \sqrt{N+1}$ :

$$
g_{00}=\frac{\tilde{g}}{\sqrt{N+1}} \sum_{j=0}^{N}\left|h_{j, 0}^{L}\right|^{2}=\frac{\tilde{g}}{\sqrt{N+1}},
$$

onde usamos a condição de normalização para o férmion, (3.82). Como $g_{00} \equiv g$ deve ser o acoplamento de gauge do MP, obtemos a relação:

$$
g=\frac{\tilde{g}}{\sqrt{N+1}} .
$$


Essa relação é análoga a que existe entre os acoplamentos de gauge 5-dimensional e os do MP, $g=g_{5} / \sqrt{\pi R}$ ou seja, $N+1$ faz o papel de "tamanho da dimensão extra" na teoria descontruída. ${ }^{8}$ De agora em diante, escreveremos os acoplamentos em função de $g$.

Usando as expressões obtidas anteriormente pra $h_{j, 0}^{L}$, (3.81) e (3.83), chegamos ao acoplamento com o m-ésimo autoestado de massa:

$$
g_{0 m}^{L}=g \sqrt{N+1} \sum_{j=0}^{N}\left(Z_{L}\right)^{2 j}\left(\frac{1-Z_{L}^{2}}{1-Z_{L}^{2(N+1)}}\right) f_{j, m}
$$

onde $Z_{L}=q^{\left(c_{L}-1 / 2\right)}$. Estamos particularmente interessados no acoplamento com o primeiro modo massivo, $m=1$, já que ele dará a maior contribuição para modificações a observáveis do MP. Assim,

$$
g_{01}^{L}=g \sqrt{N+1} \sum_{j=0}^{N}\left(Z_{L}\right)^{2 j}\left(\frac{1-Z_{L}^{2}}{1-Z_{L}^{2(N+1)}}\right) f_{j, 1} .
$$

Analogamente, para o férmion de mão direita, obtemos:

$$
g_{01}^{R}=g \sqrt{N+1} \sum_{k=0}^{N}\left(Z_{R}\right)^{2 k}\left(\frac{1-Z_{R}^{2}}{1-Z_{R}^{2(N+1)}}\right) f_{k, 1},
$$

onde $Z_{R}=q^{-\left(c_{R}+1 / 2\right)}$.

O comportamento desses acoplamentos com os parâmetros de localização $c_{L, R}$ para uma teoria de poucos sítios nos interessa, pois, assim como no modelo de RS, ele está relacionado a violação de sabor por FCNC. Como podemos ver na figura (3.3), o acoplamento de gauge para pequenos valores de $N$ se comporta

\footnotetext{
${ }^{8}$ Essa correspondência se refere apenas à definição do acoplamento. Na realidade, no limite do contínuo devemos identificar $a N \operatorname{com} \pi R$, como na fórmula (3.47).
} 


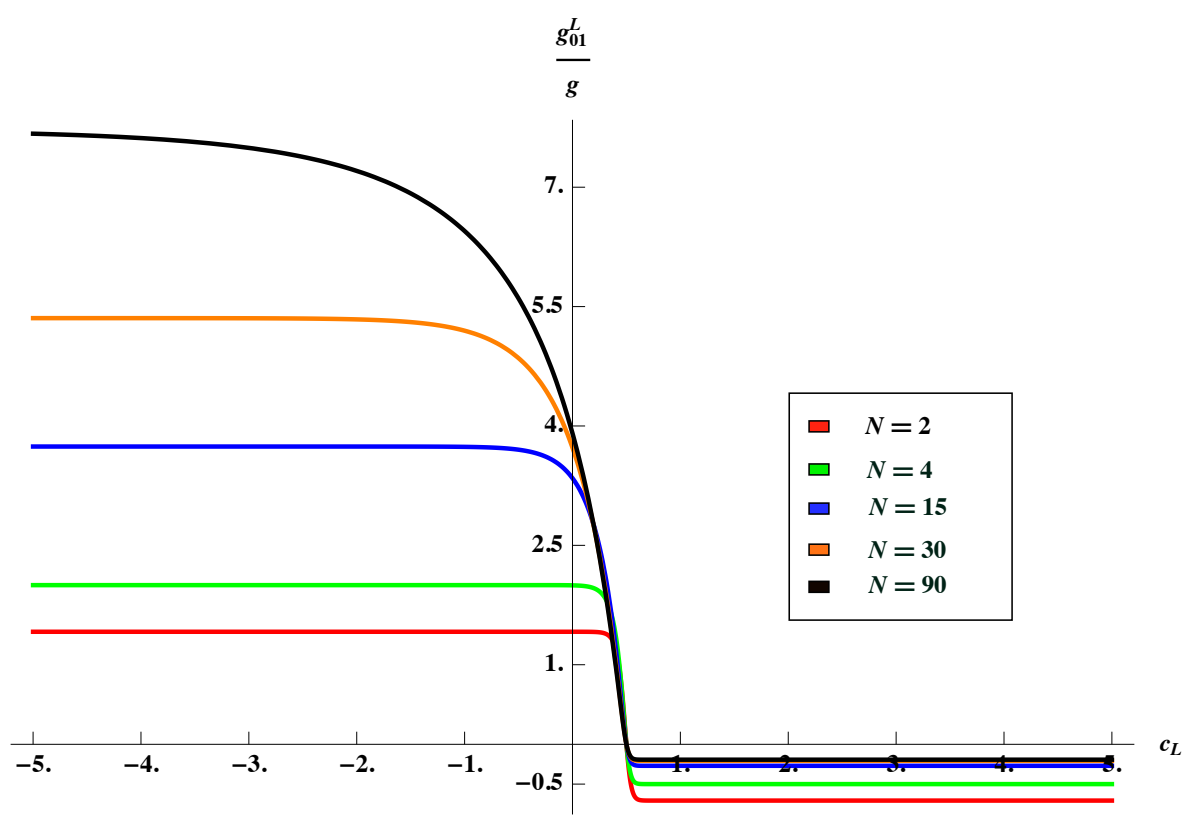

Figura 3.3: Acoplamentos de um férmion de mão esquerda com o primeiro estado excitado de um bóson de gauge, como função do parâmetro de localização $c_{L}$. Do lado esquerdo da figura e de baixo para cima, temos o gráfico para $N=2$, $N=4, N=15, N=30$ e $N=90$.

de maneira bastante diferente do acoplamento obtido no contínuo, exibido na figura (2.3) (no limite de $\mathrm{N}$ grande, ambos coincidem). É interessante notar que ocorre a rápida saturação dos acoplamentos $\operatorname{com} c_{L, R}$, formando dois platôs distintos. Como vimos na seção (2.4), a violação de sabor se faz presente devido a não universalidade dos acoplamentos de gauge. Assim, como os acoplamentos saturam-se rapidamente para poucos sítios, eles são pouco sensíveis à escolha da localização, desde que estejam todos no mesmo platô. Por esse mecanismo, esperamos suprimir as FCNC na teoria desconstruída de poucos sítios. Passaremos agora a obter os acoplamentos de Yukawa.

A maneira mais simples de incluir o setor de Higgs é assumir que existe um escalar elementar $H$ dubleto de $S U(2)_{L}$ no sítio $N$ da teoria. Isso é análogo a 
localizar o Higgs por uma função delta na brana IR no modelo de RS. No capítulo seguinte, apresentaremos um mecanismo dinâmico de localização do Higgs que permite obter modelos mais realistas.

Os acoplamentos de Yukawa serão dados por:

$$
\mathcal{L}_{\mathrm{Y}}=\bar{\psi}_{L, N} \mathrm{YH} \psi_{R, N}+\text { h.c. }
$$

Assim como no MP, a matriz de acoplamentos Y não será, em geral, diagonal na base de interação, e assim, para obter as massas, deveremos efetuar transformações unitárias independentes nos $\psi_{L, R}$. Esperamos que todos os elementos de $\mathrm{Y}$ sejam de ordem $\mathcal{O}(1)$, pois desejamos obter a hierarquia de massas dos férmions naturalmente, através de suas localizações, como ocorre no modelo de RS. Expandindo o férmion em seus auto-estados de massa, obtemos a expressão para os acoplamentos de Yukawa com o modo zero:

$$
\begin{aligned}
& \mathcal{L}_{\mathrm{Y}}=\left(Z_{L}\right)^{N}\left(Z_{R}\right)^{N}\left(\frac{1-Z_{L}^{2}}{1-Z_{L}^{2(N+1)}}\right)^{1 / 2}\left(\frac{1-Z_{R}^{2}}{1-Z_{R}^{2(N+1)}}\right)^{1 / 2} \bar{\psi}_{L, 0} \mathrm{YH} \psi_{R, 0}+\text { h.c } \\
& \equiv \bar{\psi}_{L, 0} \mathrm{Y}_{0} H \psi_{R, 0}+\text { h.c }
\end{aligned}
$$

onde definimos a matriz de Yukawa que deve corresponder a do MP:

$$
\mathrm{Y}_{0} \equiv\left(Z_{L}\right)^{N}\left(Z_{R}\right)^{N}\left(\frac{1-Z_{L}^{2}}{1-Z_{L}^{2(N+1)}}\right)^{1 / 2}\left(\frac{1-Z_{R}^{2}}{1-Z_{R}^{2(N+1)}}\right)^{1 / 2} \mathrm{Y}
$$

Embora os acoplamentos Y sejam não hierárquicos, podemos obter a hierarquia de massas apenas escolhendo os parâmetros de localização $c_{L, R}$, de maneira análoga ao que ocorre no modelo de RS. Dado que os acoplamentos de gauge 
para poucos sítios saturam-se rapidamente com a localização, esperamos que seja possível obter essa hierarquia sem gerar as grandes FCNC que ocorrem no modelo de RS.

Na seção seguinte exploraremos o espaço de parâmetros da teoria de cinco sítios, e exibiremos soluções que geram a hierarquia de massas dos quarks e a matriz de mistura CKM, com acoplamentos de gauge quase universais e que satisfazem também os vínculos impostos pelos parâmetros de precisão eletrofraca. Esses estudos levaram a publicação do trabalho [31].

\subsection{Violação de Sabor}

Para estudar quantitativamente as questões da violação de sabor e dos parâmetros de precisão eletrofraca, trabalhamos em um modelo de cinco sítios $(N=$ 4). Esse valor de $N$ não é especial, mas é tomado apenas para tornar a discussão concreta. Enquanto nos mantivermos em um modelo de poucos sítios, as características serão qualitativamente semelhentes.

Por simplicidade, tomaremos as matrizes $\mathrm{Y}_{u, d}$ reais. Sempre que uma fase for importante, por exemplo, na violação CP no setor de Kaons, assumiremos o valor que maximiza a contribuição. Efetuamos uma varredura do espaço de parâmetros, tomando as localizações entre -1,5 e 1,5 e os acoplamentos de Yukawa entre -3 e 3, buscando soluções que resultam na hierarquia de massas e ângulos de mistura dos quarks, com acoplamentos de gauge quase universais para suprimir as FCNC. Com três dubletos de quarks de mão esquerda, três quarks tipo up e três tipo down de mão direita, temos dezoito entradas das matrizes de Yukawa $\mathrm{Y}_{u, d}$ e mais nove localizações a determinar. Esses parâmetros não são completa- 
mente independentes pois existem classes de equivalência no espaço de parâmetros cujos pontos definem as mesmas massas e ângulos de mistura [52, 48, 53]. Isso é intuitivamente claro se pensarmos que, para uma massa fixa, uma variação da localização dos dubletos de mão esquerda sempre pode ser compensada por uma variação na localização dos quarks de mão direita, e vice-versa. Analogamente, se reescalarmos as funções de onda dos quarks por um fator $\eta$, podemos compensar dividindo o acoplamento de Yukawa pelo mesmo fator, $Y \rightarrow Y / \eta$. Nosso propósito não é classificar essas equivalências, mas apenas exibir pontos particulares do espaço de parâmetros que tem as características desejadas.

Examinando o gráfico dos acoplamentos para $N=4$ (figura (3.4)), temos dois platôs onde os acoplamentos são universais, minimizando portanto a violação de sabor, com uma pequena região de transição aproximadamente entre $0.15<c_{L}<0.6$ (ou $-0.15>c_{R}>-0.6$ ). Para férmions no platô inferior a localização é próxima ao sítio $j=N(\mathrm{UV})$ enquanto que o platô superior corresponde a localização no sítio $j=0$ (IR). Comparando com o caso contínuo (figura (2.3)), vemos que no modelo de RS só existe um platô, ou seja, só obtemos universalidade para localizações no UV. As soluções obtidas podem ser classificadas de acordo com as características qualitativas em dois casos, que chamaremos de caso A e caso B.

Uma solução típica do caso A é exibida nas figuras (3.5), (3.6) e (3.7). Os pontos azuis, verdes e vermelhos representam diferentes sabores de quarks na base de interação. Nesse caso, os quarks de mão esquerda estão localizados no IR e os de mão direita no UV. Vemos que a violação de sabor é mínima, pois mesmo para localizações bastante diferentes, os acoplamentos de gauge se encontram todos no mesmo platô, com exceção de um dos quarks up de mão direita, o que é esperado, 


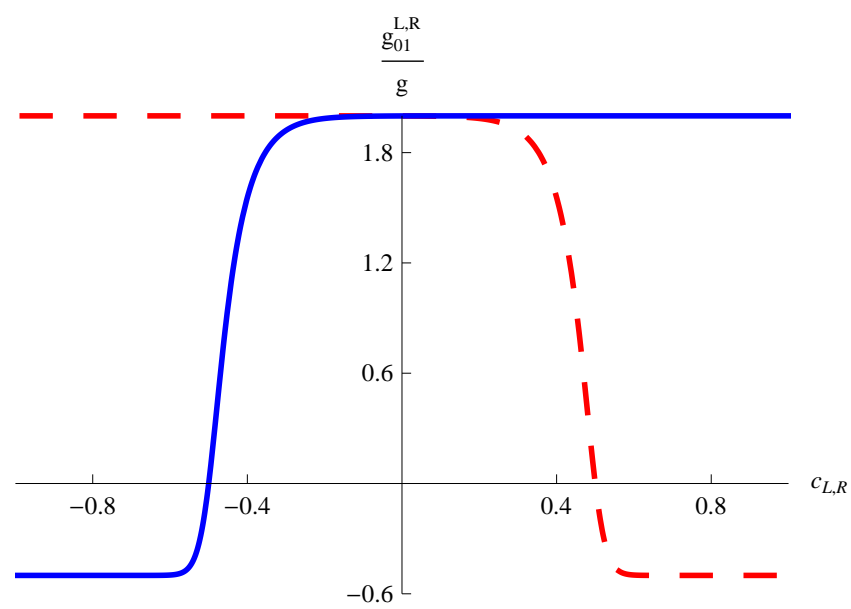

Figura 3.4: Acoplamento de gauge dos modos zero com o primeiro modo massivo de um bóson de gauge para $N=4$, em unidades do acoplamento do MP. Em vermelho tracejado (azul) temos o acoplamento de mão esquerda (direita) em função da localização $c_{L(R)}$.

pois necessitamos obter a grande massa do quark top. Como tanto os quarks de mão esquerda quanto os de mão direita tipo down tem acoplamentos universais, nesse caso não existe violação de sabor no setor down, e assim os vínculos da

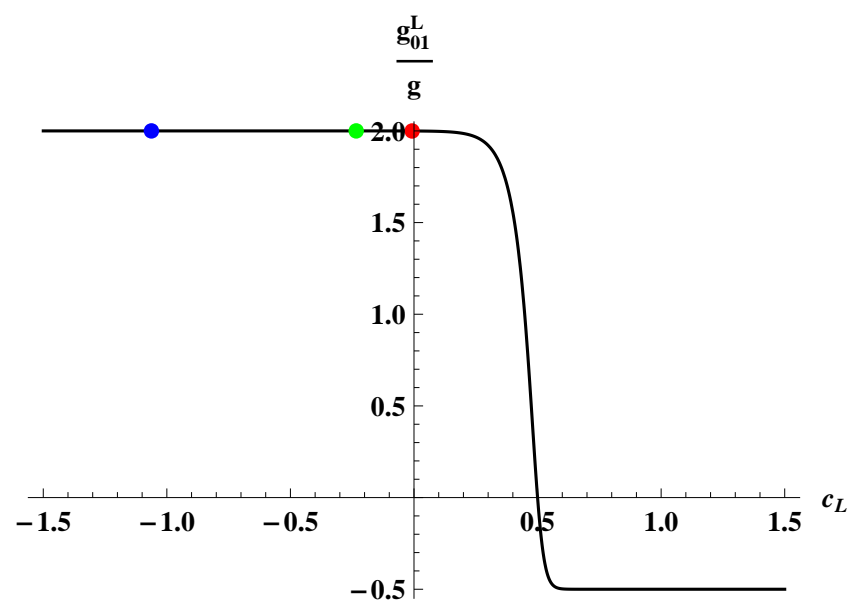

Figura 3.5: Acoplamento de gauge dos modos zero dos dubletos de mão-esquerda de quarks com o primeiro modo massivo de um bóson de gauge para $N=5$, em unidades do acoplamento do MP. Os pontos azul, verde e vermelho representam diferentes sabores na base de interação. A figura corresponde ao caso $\mathbf{A}$. 


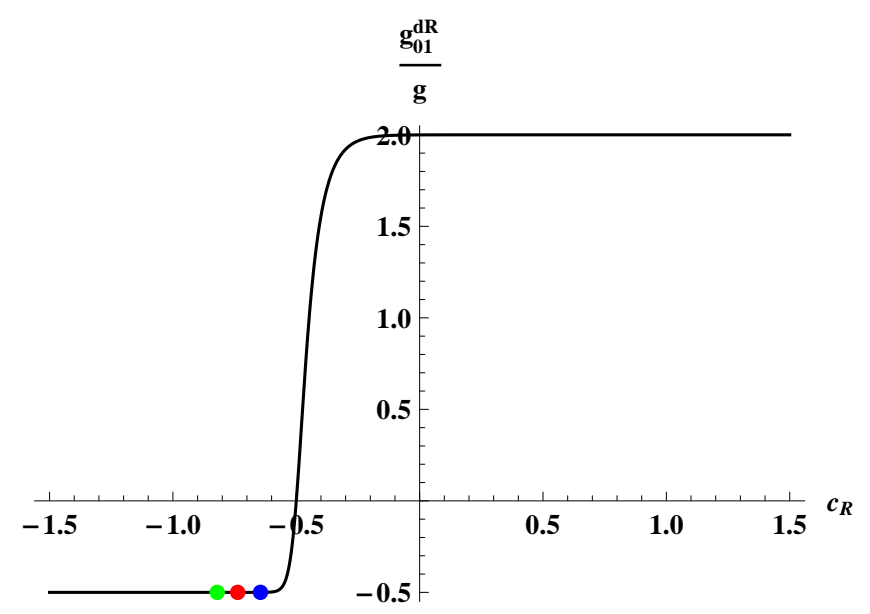

Figura 3.6: Acoplamento de gauge dos modos zero dos singletos de mão-direita de quarks tipo down com o primeiro modo massivo de um bóson de gauge para $N=5$, em unidades do acoplamento do MP. Os pontos azul, verde e vermelho representam diferentes sabores na base de interação. A figura corresponde ao caso A.

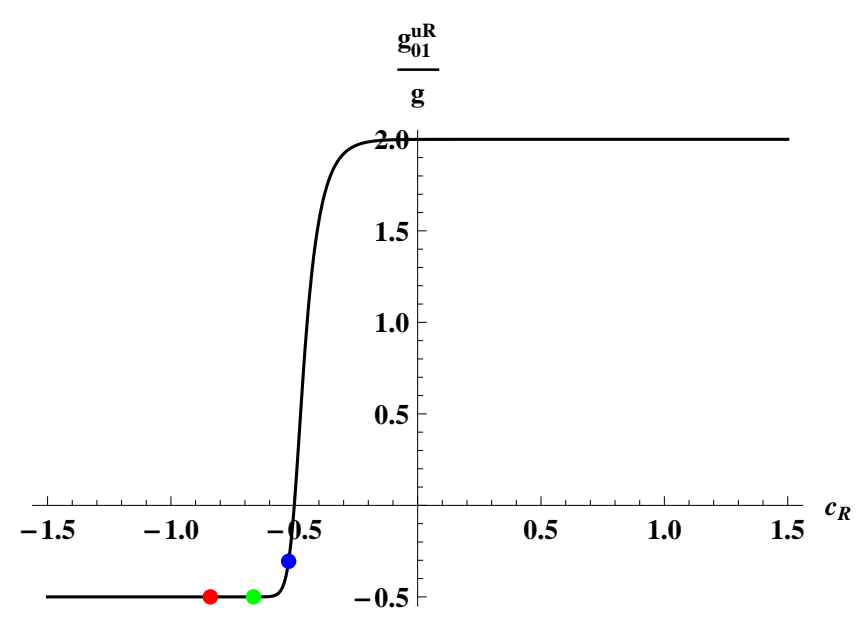

Figura 3.7: Acoplamento de gauge dos modos zero dos singletos de mão-direita de quarks tipo up com o primeiro modo massivo de um bóson de gauge para $N=5$, em unidades do acoplamento do MP. Os pontos azul, verde e vermelho representam diferentes sabores na base de interação. A figura corresponde ao caso A. 
física de Kaons que exigem $M_{K K} \sim 30 \mathrm{TeV}$ no contínuo [57] são evitados, como veremos a seguir. Existe apenas uma violação de sabor pequena no setor up, de maneira que os vínculos mais restritivos virão da mistura de mésons $D^{0}$.

Para o caso B, uma das soluções é mostrada nas figuras (3.8), (3.9) e (3.10), todos os quarks estão localizados no platô UV, com excessão de um dos dubletos de mão esquerda e um dos quarks up de mão direita, novamente devido a massa do top. Esse caso é mais parecido com o que ocorre em modelos de dimensões extras curvas. Nesse caso, a violação de sabor é visivelmente maior do que no caso A e, em particular, a não universalidade dos acoplamentos dos dubletos induz violação de sabor para os quarks tipo down. No entanto, como veremos, as contribuições para os parâmetros de precisão eletrofraca são menores.

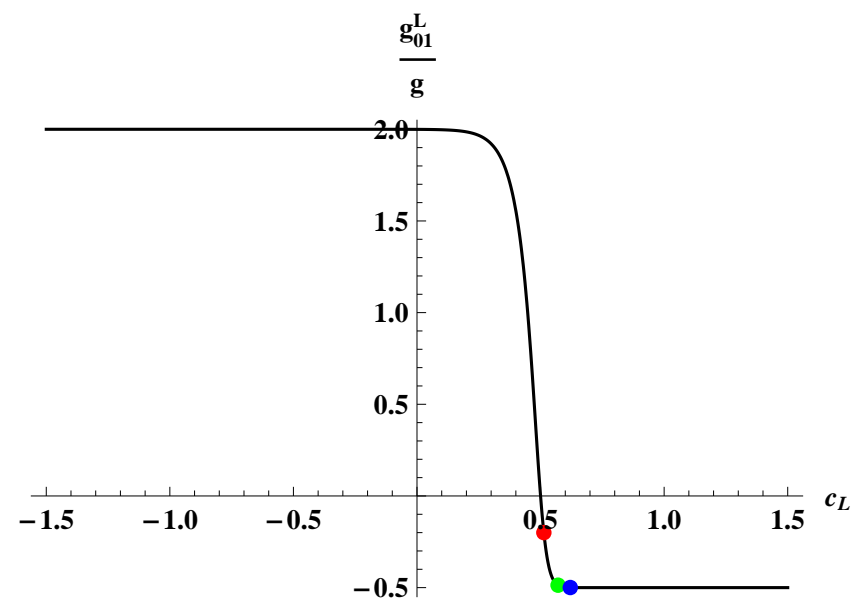

Figura 3.8: Acoplamento de gauge dos modos zero dos dubletos de mão-esquerda de quarks com o primeiro modo massivo de um bóson de gauge para $N=5$, em unidades do acoplamento do MP. Os pontos azul, verde e vermelho representam diferentes sabores na base de interação. A figura corresponde ao caso $\mathbf{B}$.

Se a nossa intenção é apenas resolver os problemas de hierarquia associados ao setor eletrofraco, não é estritamente necessário assumir que as interações fortes se propaguem no diagrama quiver, mas vamos considerar esse caso pois os 


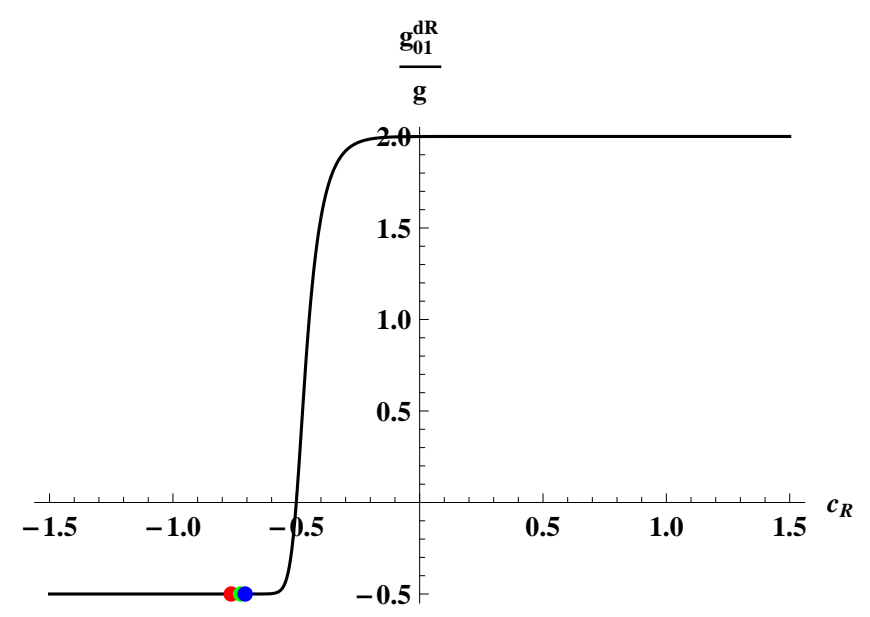

Figura 3.9: Acoplamento de gauge dos modos zero dos singletos de mão-direita de quarks tipo down com o primeiro modo massivo de um bóson de gauge para $N=5$, em unidades do acoplamento do MP. Os pontos azul, verde e vermelho representam diferentes sabores na base de interação. A figura corresponde ao caso B.

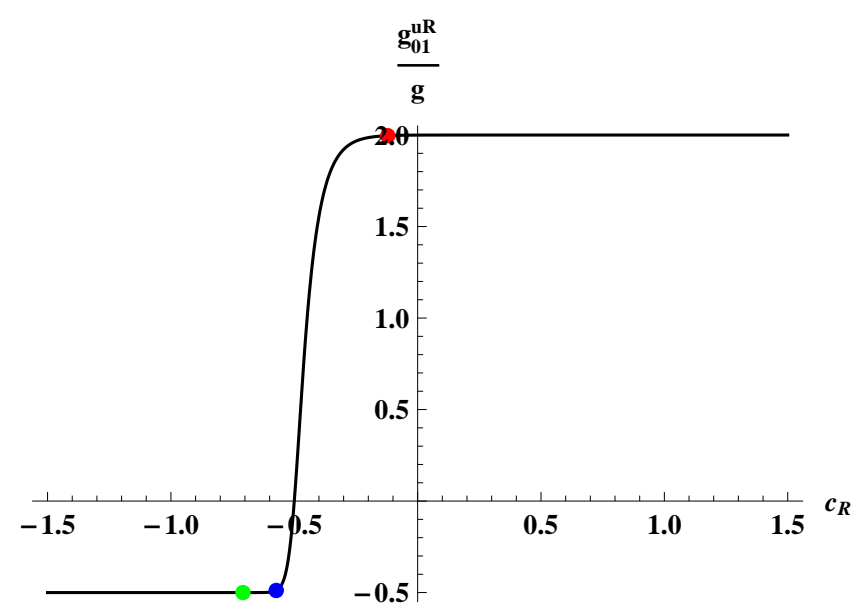

Figura 3.10: Acoplamento de gauge dos modos zero dos singletos de mão-direita de quarks tipo up com o primeiro modo massivo de um bóson de gauge para $N=5$, em unidades do acoplamento do MP. Os pontos azul, verde e vermelho representam diferentes sabores na base de interação. A figura corresponde ao caso B.

vínculos mais restritivos virão de assumir que a violação de sabor é mediada pelo bóson de gauge de $S U(3)_{c}$ e são esses vínculos que podem ser diretamente comparados com os obtidos para os modelos em $A d S_{5}$. 
Para estudar quantitativamente os efeitos de violação de sabor, precisamos efetuar a rotação para a base de autoestados de massa na matriz de acoplamentos, segundo a expressão:

$$
G_{L}^{U} \equiv L_{U}^{-1}\left(\begin{array}{ccc}
g_{u_{L}} & 0 & 0 \\
0 & g_{c_{L}} & 0 \\
0 & 0 & g_{t_{L}}
\end{array}\right) L_{U}
$$

onde $L_{U}$ é a matriz unitária que roda para base de autoestados de massa os quarks up de mão esquerda e os acoplamentos $g_{u_{L}}, g_{c_{L}}$ e $g_{t_{L}}$ são os definidos por (3.91). Analogamente, definimos matrizes de rotação para $R_{U}$ para os quarks up de mão direita, e $R_{D}$ e $L_{D}$ para o setor down de mão direita e esquerda, respectivamente, como na equação (2.80). Os acoplamentos responsáveis pelas transições que violam sabor serão os elementos fora da diagonal dessas matrizes. Para as duas soluções exibidas, eles estão nas tabelas (3.1) para o caso A e (3.2) para o caso B. Esses valores devem ser comparados com o acoplamento forte na escala de massas do primeiro modo massivo, $\sqrt{4 \pi \alpha_{S}\left(M^{(1)}\right)} \approx 1$.

\begin{tabular}{|c|c|c|c|c|c|}
\hline & $\mathrm{L}$ & $\mathrm{R}$ & & $\mathrm{L}$ & $\mathrm{R}$ \\
\hline$\left|G^{u, c}\right|$ & $1.1 \times 10^{-5}$ & $2.2 \times 10^{-8}$ & $\left|G^{d, s}\right|$ & $5.7 \times 10^{-5}$ & $1.6 \times 10^{-9}$ \\
\hline$\left|G^{u, t}\right|$ & $2.0 \times 10^{-4}$ & $2.3 \times 10^{-6}$ & $\left|G^{d, b}\right|$ & $1.9 \times 10^{-4}$ & $2.1 \times 10^{-8}$ \\
\hline$\left|G^{c, t}\right|$ & $5.5 \times 10^{-6}$ & $6.8 \times 10^{-4}$ & $\left|G^{s, b}\right|$ & $5.9 \times 10^{-5}$ & $2.5 \times 10^{-6}$ \\
\hline
\end{tabular}

Tabela 3.1: Caso A: Valores dos acoplamentos não diagonais dos quarks ao primeiro estado excitado do gluon. 


\begin{tabular}{|c|c|c|c|c|c|}
\hline & $\mathrm{L}$ & $\mathrm{R}$ & & $\mathrm{L}$ & $\mathrm{R}$ \\
\hline$\left|G^{u, c}\right|$ & $2.8 \times 10^{-3}$ & $2.9 \times 10^{-4}$ & $\left|G^{d, s}\right|$ & $5.7 \times 10^{-4}$ & $6.5 \times 10^{-6}$ \\
\hline$\left|G^{u, t}\right|$ & $4.2 \times 10^{-3}$ & $2.9 \times 10^{-3}$ & $\left|G^{d, b}\right|$ & $5.9 \times 10^{-3}$ & $5.0 \times 10^{-5}$ \\
\hline$\left|G^{c, t}\right|$ & $3.3 \times 10^{-2}$ & $1.8 \times 10^{-1}$ & $\left|G^{s, b}\right|$ & $6.7 \times 10^{-3}$ & $1.2 \times 10^{-4}$ \\
\hline
\end{tabular}

Tabela 3.2: Caso B: Valores dos acoplamentos não diagonais dos quarks ao primeiro estado excitado do gluon.

A troca do primeiro modo massivo no canal s resulta na Hamiltoniana de interação (válida para momentos $q \ll M_{G}$, onde $M_{G}$ é a massa do estado):

$$
\mathcal{H}_{\mathrm{eff}}=\frac{1}{2 M_{G}^{2}}\left(\bar{\psi}_{\alpha}^{i} \gamma_{\mu}\left(G_{L}^{i j} P_{L}+G_{R}^{i j} P_{R}\right) T_{\alpha \beta}^{a} \psi_{\beta}^{j}\right)\left(\bar{\psi}_{\delta}^{k} \gamma^{\mu}\left(G_{L}^{i j} P_{L}+G_{R}^{k l} P_{R}\right) T_{\delta \lambda}^{a} \psi_{\lambda}^{l}\right)
$$

onde $T^{a}$ são os geradores de $S U(3), P_{L, R}=\left(1 \mp \gamma^{5}\right) / 2$ são os projetores quirais, $\alpha, \beta, \delta, \lambda$ são índices de cor e $i, j, k, l$ de sabor. Podemos simplificar essa expressão utilizando a relação de completeza para os geradores de $S U(N)$ :

$$
\sum_{a=1}^{N^{2}-1} T_{\alpha \beta}^{a} T_{\delta \lambda}^{a}=\frac{1}{2}\left(\delta_{\alpha \lambda} \delta_{\beta \delta}-\frac{1}{N} \delta_{\alpha \beta} \delta_{\delta \lambda}\right)
$$

E as identidades de Fierz:

$$
\begin{aligned}
& \bar{\psi}_{L}^{1} \gamma^{\mu} \psi_{L}^{2} \bar{\psi}_{L}^{3} \gamma_{\mu} \psi_{L}^{4}=\bar{\psi}_{L}^{1} \gamma^{\mu} \psi_{L}^{4} \bar{\psi}_{L}^{3} \gamma_{\mu} \psi_{L}^{2}, \\
& \bar{\psi}_{R}^{1} \gamma^{\mu} \psi_{R}^{2} \bar{\psi}_{R}^{3} \gamma_{\mu} \psi_{R}^{4}=\bar{\psi}_{R}^{1} \gamma^{\mu} \psi_{R}^{4} \bar{\psi}_{R}^{3} \gamma_{\mu} \psi_{R}^{2}, \\
& \bar{\psi}_{L}^{1} \gamma^{\mu} \psi_{L}^{2} \bar{\psi}_{R}^{3} \gamma_{\mu} \psi_{R}^{4}=-2 \bar{\psi}_{L}^{1} \psi_{R}^{4} \bar{\psi}_{R}^{3} \psi_{L}^{2} .
\end{aligned}
$$

Chegamos assim a Hamiltoniana efetiva para transições com $\Delta F=2(i=k$, 
$j=l):$

$$
\begin{aligned}
\mathcal{H}_{\mathrm{eff}}= & \frac{1}{M_{G}^{2}}\left[\frac{1}{6} G_{L}^{i j} G_{L}^{i j}\left(\bar{\psi}_{L}^{i \alpha} \gamma^{\mu} \psi_{L \alpha}^{j}\right)\left(\bar{\psi}_{L}^{i \beta} \gamma^{\mu} \psi_{L \beta}^{j}\right)+(\mathrm{L} \leftrightarrow \mathrm{R})\right. \\
& \left.-G_{L}^{i j} G_{R}^{i j}\left(\left(\bar{\psi}_{R}^{i \alpha} \psi_{L \alpha}^{j}\right)\left(\bar{\psi}_{L}^{i \beta} \psi_{R \beta}^{j}\right)-\frac{1}{3}\left(\bar{\psi}_{R}^{i \alpha} \psi_{L \beta}^{j}\right)\left(\bar{\psi}_{L}^{i \alpha} \psi_{R \beta}^{j}\right)\right)\right]
\end{aligned}
$$

onde $M_{G}$ é a massa do primeiro modo do gluon. Os coeficientes de (3.100) devem ser identificados com os coeficientes de Wilson da Hamiltoniana efetiva a baixas energias na escala $\Lambda=M_{G}$. Seguindo a convenção adotada na literatura [72], as contribuições resultam nos coeficientes:

$$
C_{M}^{1}\left(M_{G}\right)=\frac{1}{6} \frac{\left(G_{L}^{i j}\right)^{2}}{M_{G}^{2}}, \quad C_{M}^{4}\left(M_{G}\right)=\frac{G_{L}^{i j} G_{R}^{i j}}{M_{G}^{2}}, \quad C_{M}^{5}\left(M_{G}\right)=\frac{G_{L}^{i j} G_{R}^{i j}}{3 M_{G}^{2}}
$$

onde $M=K, D, B_{d}, B_{s}$ se refere aos mésons que participam da transição (por exemplo, se $M=K$, então $i, j=d, s$ ). Os ajustes feitos pela colaboração UTFit [72] resultam então em vínculos para os coeficientes de Wilson (para o caso dos Kaons, utilizamos vínculos mais recentes vindos de cálculos na rede [73]), e consequentemente, para a massa $M_{G}$, uma vez que fixamos os acoplamentos. Alguns cuidados são necessários na interpretação desses vínculos. Em geral o coeficiente de Wilson terá a forma genérica $C^{i}=F^{i} / \Lambda^{2}$, onde os $F^{i}$ são funções dos acoplamentos e possíveis fatores de loop e $\Lambda$ é o corte da teoria efetiva, que dá a escala da física nova. O vínculo mais conservador vem de tomar os $F^{i}=1$ e, nesse caso, o vínculo sobre o coeficiente se traduz diretamente num valor mínimo para o corte $\Lambda$. Por outro lado, em nosso modelo, os acoplamentos serão naturalmente suprimidos, e assim o corte, que corresponde à massa $M_{G}$, poderá ser consideravelmente mais baixo. Nos casos em que a escala assumida no vín- 
culo e a escala da massa $M_{G}$ são muito diferentes, é necessário ainda considerar correções que vem da evolução sob o grupo de renormalização dos coeficientes de Wilson. De maneira simplificada, os operadores serão corrigidos por fatores da forma $\eta_{i} \sim\left(\alpha_{s}(\Lambda) / \alpha_{s}\left(M_{G}\right)\right)^{a_{i}}$, onde $\alpha_{s}(\mu)$ é a constante de acoplamento forte e a potência $a_{i}$ é diferente para cada $C_{i}[74]$.

\begin{tabular}{cccc}
\hline \hline Parâmetro & $\begin{array}{c}\text { Intervalo de } 95 \% \\
\text { de confiança }\left(\mathrm{GeV}^{-2}\right)\end{array}$ & $\begin{array}{c}\text { Limite inferior } \\
\text { para } \Lambda(\mathrm{TeV})\end{array}$ & $\begin{array}{c}\text { Vínculo para } \\
M_{G}(\mathrm{TeV})\end{array}$ \\
\hline $\operatorname{Re} C_{K}^{1}$ & {$[-9.6,9.6] \cdot 10^{-13}$} & $1.0 \cdot 10^{3}$ & 0.2 \\
$\operatorname{Re} C_{K}^{4}$ & {$[-3.6,3.6] \cdot 10^{-15}$} & $17 \cdot 10^{3}$ & 0.1 \\
$\operatorname{Re} C_{K}^{5}$ & {$[-1.0,1.0] \cdot 10^{-14}$} & $10 \cdot 10^{3}$ & 0.1 \\
\hline $\operatorname{Im} C_{K}^{1}$ & {$[-2.6,2.8] \cdot 10^{-15}$} & $1.9 \cdot 10^{4}$ & 2.6 \\
$\operatorname{Im} C_{K}^{4}$ & {$[-4.1,3.6] \cdot 10^{-18}$} & $49 \cdot 10^{4}$ & 3.0 \\
$\operatorname{Im} C_{K}^{5}$ & {$[-1.2,1.1] \cdot 10^{-17}$} & $29 \cdot 10^{4}$ & 1.0 \\
\hline \hline$\left|C_{D}^{1}\right|$ & $<7.2 \cdot 10^{-13}$ & $1.2 \cdot 10^{3}$ & 1.0 \\
$\left|C_{D}^{4}\right|$ & $<4.8 \cdot 10^{-14}$ & $4.6 \cdot 10^{3}$ & 2.9 \\
$\left|C_{D}^{5}\right|$ & $<4.8 \cdot 10^{-13}$ & $1.4 \cdot 10^{3}$ & 0.5 \\
\hline \hline$\left|C_{B_{d}}^{1}\right|$ & $<2.3 \cdot 10^{-11}$ & $0.21 \cdot 10^{3}$ & 0.3 \\
$\left|C_{B_{d}}^{4}\right|$ & $<2.1 \cdot 10^{-13}$ & $2.2 \cdot 10^{3}$ & 0.3 \\
$\left|C_{B_{d}}^{5}\right|$ & $<6.0 \cdot 10^{-13}$ & $1.3 \cdot 10^{3}$ & 0.1 \\
\hline \hline$\left|C_{B_{s}}^{1}\right|$ & $<1.1 \cdot 10^{-9}$ & 30 & 0.1 \\
$\left|C_{B_{s}}^{4}\right|$ & $<1.6 \cdot 10^{-11}$ & 250 & 0.1 \\
$\left|C_{B_{s}}^{5}\right|$ & $<4.5 \cdot 10^{-11}$ & 150 & 0.03 \\
\hline \hline
\end{tabular}

Tabela 3.3: Intervalo de $95 \%$ de confiança para $C(\Lambda)$ e correspondentes vínculos para a escala de nova física $\Lambda$, obtidos das referências $[72,73]$. A última coluna corresponde ao vínculo para a massa do primeiro estado massivo do glúon para o caso B descrito no texto.

Os resultados estão sumarizados na tabela (3.3). Na segunda coluna, temos os valores obtidos do ajuste para os coeficientes de Wilson, em um intervalo de $95 \%$ de confiança. A terceira coluna dá a escala de física nova obtida se assumirmos $C(\Lambda) \sim 1 / \Lambda^{2}$ e a quarta coluna o vínculo obtido para a massa do octeto de cor no caso B. Comparando as tabelas (3.1) e (3.2), vemos que os acoplamentos fora da 
diagonal no caso A são ordens de grandeza menores que no caso B, e assim, não existe vínculo significativo nesse caso.

Como esperado, no setor down vemos que o vínculo mais restritivo para o caso B vem da física de Kaons. O vínculo que vem de $\operatorname{Im} C_{K}^{4}$ resulta em:

$$
M_{G}>3 \mathrm{TeV}
$$

Outro vínculo similar vem de $\operatorname{Im} C_{K}^{1}$, que resulta em $M_{G}>2.6 \mathrm{TeV}$. No setor up, o vínculo sobre $\left|C_{D}^{4}\right|$ requer $M_{G}>2.9 \mathrm{TeV}$. Nesse caso, a violação de sabor vem da localização do quark top próxima ao sítio $N$ que é necessária para gerar a massa do top. Em comparação, vimos que os vínculos similares para o modelo de RS requerem que o primeiro modo de $\mathrm{KK}$ do gluon tenha massa $M_{K K} \gtrsim 30 \mathrm{TeV}$ [57].

Vemos então que a teoria desconstruída com poucos sítios permite manter as soluções para os problemas de hierarquia de gauge e de massas dos férmions, que são características atraentes do modelo de RS, com a possibilidade, no entanto, de evitar as grandes FCNC geradas pela não universalidade dos acoplamentos. Passamos a examinar os vínculos que vêm dos parâmetros de precisão eletrofraca.

\subsection{Parâmetros de Precisão Eletrofraca}

Uma fonte importante de vínculos para teorias além do MP é dada pelas medidas de precisão da teoria eletrofraca $[3,4]$. Usualmente, as correções mais importantes são as que afetam a auto-energia dos bósons de gauge de maneira universal, chamadas correções oblíquas, que têm a forma do diagrama da figura (3.11), onde somamos as contribuições dos processos irredutíveis de uma partícula (one- 


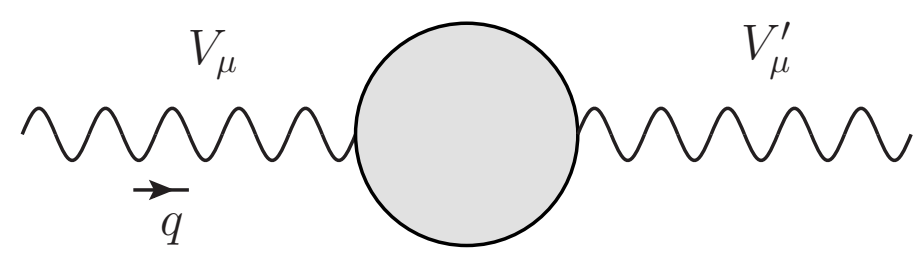

Figura 3.11: Auto-energia de um bóson de gauge $V_{\mu}$. A região cinza representa a soma das contribuições 1PI. O momento carregado pelo bóson é $q_{\mu}$.

particle-irreducible - 1PI). ${ }^{9}$ Antes de discutir as correções oblíquas propriamente, vamos relembrar a interpretação da auto-energia, ou função de polarização do vácuo.

A auto-energia pode ser escrita como:

$$
i \Pi_{V V^{\prime}}^{\mu \nu}\left(q^{2}\right) \equiv i\left[\Pi_{V V^{\prime}}\left(q^{2}\right) g^{\mu \nu}-\Delta_{V V^{\prime}}\left(q^{2}\right) q^{\mu} q^{\nu}\right]
$$

onde em geral $V \neq V^{\prime}$. Se assumirmos que o bóson se acopla a férmions leves, o termo $\Delta_{V V^{\prime}}\left(q^{2}\right)$ pode ser desconsiderado, pela equação de Dirac:

$$
i q_{\mu} J^{\mu}=\bar{\psi} i q \psi=\bar{\psi} m \psi \simeq 0
$$

Podemos ressomar as inserções de $\Pi_{V V^{\prime}}\left(q^{2}\right)$ no propagador (gauge de Feynman):

$$
\begin{aligned}
& \frac{-i g_{\mu \nu}}{q^{2}-M_{V}^{2}}+\frac{-i g_{\mu \alpha}}{q^{2}-M_{V}^{2}} i \Pi_{V V}\left(q^{2}\right) g^{\alpha \beta} \frac{-i g_{\beta \nu}}{q^{2}-M_{V}^{2}}+\cdots \\
& =\frac{-i g_{\mu \nu}}{q^{2}-M_{V}^{2}}\left(1+\frac{\Pi_{V V}}{q^{2}-M_{V}^{2}}+\left(\frac{\Pi_{V V}}{q^{2}-M_{V}^{2}}\right)^{2}+\cdots\right) \\
& =\frac{-i g_{\mu \nu}}{q^{2}-M_{V}^{2}} \frac{1}{1-\frac{\Pi_{V V}}{q^{2}-M_{V}^{2}}}=\frac{-i g_{\mu \nu}}{q^{2}-M_{V}^{2}-\Pi_{V V}\left(q^{2}\right)} .
\end{aligned}
$$

\footnotetext{
${ }^{9}$ Um exemplo importante de correções não universais são as que afetam o vértice $Z b \bar{b}$.
} 
Expandindo $\Pi_{V V^{\prime}}\left(q^{2}\right)=\Pi_{V V}\left(M_{V}^{2}\right)+\Pi_{V V}^{\prime}\left(M_{V}^{2}\right)\left(q^{2}-M_{V}^{2}\right)+\cdots$, obtemos as correções para o polo e resíduo do propagador:

$$
\frac{-i g_{\mu \nu}}{q^{2}-M_{V}^{2}-\Pi_{V V}\left(q^{2}\right)} \simeq \frac{-i g_{\mu \nu}\left(1+\Pi_{V V}^{\prime}\left(M_{V}^{2}\right)\right)}{q^{2}-M_{V}^{2}-\Pi_{V V}\left(M_{V}^{2}\right)}
$$

Portanto a massa é corrigida por $\delta M_{V}^{2}=\Pi_{V V}\left(M_{V}^{2}\right)$ e o resíduo por $\delta Z=\Pi_{V V}^{\prime}\left(M_{V}^{2}\right)$. Notamos que $\Pi_{V V}$ em geral não é uma função real, e sua parte imaginária contribuirá para a largura da partícula [1]. Para o fóton, a auto-energia é transversa $\left(q_{\mu} \Pi_{\gamma \gamma}^{\mu \nu}\left(q^{2}\right)=0\right)$ pelas identidades de Ward e o polo não é deslocado [1]. Na Lagrangiana efetiva que considera as correções quânticas vindas de $\Pi_{V V^{\prime}}\left(q^{2}\right)$, podemos escrever:

$$
\mathcal{L}_{V}=-\frac{1}{4}\left(1+\Pi_{V V}^{\prime}\right) F_{\mu \nu} F^{\mu \nu}+\frac{1}{2}\left(M_{V}^{2}+\Pi_{V V}\right) V_{\mu} V^{\mu}
$$

onde $F_{\mu \nu}$ é o tensor intensidade de campo de $V_{\mu}$.

No MP, temos quatro funções de polarização de vácuo para o setor eletrofraco, $\Pi_{\gamma \gamma}, \Pi_{W W}, \Pi_{Z Z}$, e $\Pi_{\gamma Z}$. Um conjunto equivalente, que podemos adotar por conveniência, é dado por [1]:

$$
\begin{aligned}
& \Pi_{\gamma \gamma}=e^{2} \Pi_{Q Q}, \\
& \Pi_{W W}=g^{2} \Pi_{11}, \\
& \Pi_{Z Z}=\left(g^{2}+g^{\prime 2}\right)\left[\Pi_{33}-2 s_{w}^{2} \Pi_{3 Q}+s_{w}^{4} \Pi_{Q Q}\right], \\
& \Pi_{\gamma Z}=g g^{\prime}\left[\Pi_{3 Q}-s_{w}^{2} \Pi_{Q Q}\right],
\end{aligned}
$$


onde $e^{2}=g^{2} g^{\prime 2} /\left(g^{2}+g^{\prime 2}\right), s_{w}^{2}=g^{\prime 2} /\left(g^{2}+g^{\prime 2}\right)$ define o seno do ângulo de Weinberg, $1,2,3$ denota os geradores de $S U(2)_{L}$ e $Q=T_{3}+Y$ é o operador carga elétrica. As contribuições da física nova para essas auto-energias afetam quantidades observáveis, sujeitas a medidas de grande precisão. Assumindo que a escala de física nova $\Lambda$ é muito maior do que o momento $q^{2}$ típico dos processos, podemos utilizar a aproximação $\Pi_{V V^{\prime}}\left(q^{2}\right) \simeq \Pi_{V V^{\prime}}(0)+\Pi_{V V^{\prime}}^{\prime}(0) q^{2}$, obtendo:

$$
\begin{aligned}
& \Pi_{\gamma \gamma}\left(q^{2}\right) \simeq q^{2} \Pi_{\gamma \gamma}^{\prime}(0) \\
& \Pi_{\gamma Z}\left(q^{2}\right) \simeq q^{2} \Pi_{\gamma Z}^{\prime}(0) \\
& \Pi_{W W}\left(q^{2}\right) \simeq \Pi_{W W}(0)+q^{2} \Pi_{W W}^{\prime}(0) \\
& \Pi_{Z Z}\left(q^{2}\right) \simeq \Pi_{Z Z}(0)+q^{2} \Pi_{Z Z}^{\prime}(0)
\end{aligned}
$$

onde lembramos que $\Pi_{\gamma \gamma}(0)=\Pi_{\gamma Z}(0)=0$. Temos portanto, seis parâmetros, três dos quais podem ser determinados pelas condições de renormalização dos parâmetros do MP. Usualmente são tomados os que são medidos com maior precisão: a constante de estrutura fina, $\alpha$, a constante de Fermi, $G_{F}$, e a massa do bóson $Z, M_{Z}$. Os outros três fornecem então previsões mensuráveis. Nesse caso, um conjunto de observáveis útil para parametrizar as correções oblíquas são os 
parâmetros S, T e U de Peskin-Takeuchi [75], definidos por:

$$
\begin{aligned}
S & =\frac{16 \pi}{g^{2}+g^{\prime 2}}\left(\Pi_{Z Z}^{\prime}(0)-\frac{g^{2}-g^{\prime 2}}{g g^{\prime}} \Pi_{\gamma Z}^{\prime}(0)-\Pi_{\gamma \gamma}^{\prime}(0)\right) \\
& =16 \pi\left(\Pi_{33}^{\prime}(0)-\Pi_{3 Q}^{\prime}(0)\right), \\
T & =\frac{4 \pi}{e^{2}}\left(\frac{\Pi_{W W}(0)}{M_{W}^{2}}-\frac{\Pi_{Z Z}(0)}{M_{Z}^{2}}\right) \\
& =\frac{16 \pi}{v_{E W}^{2} e^{2}}\left(\Pi_{11}(0)-\Pi_{33}(0)\right), \\
U & =\frac{16 \pi}{g^{2}}\left(\Pi_{W W}^{\prime}(0)-c_{w}^{2} \Pi_{Z Z}^{\prime}(0)-2 s_{w} c_{w} \Pi_{\gamma Z}^{\prime}(0)-s_{w}^{2} \Pi_{\gamma \gamma}^{\prime}(0)\right) \\
& =16 \pi\left(\Pi_{11}^{\prime}(0)-\Pi_{33}^{\prime}(0)\right),
\end{aligned}
$$

onde $v_{E W}=246 \mathrm{GeV}$ é a escala da quebra da simetria eletrofraca. Veremos a seguir que S e T são gerados na Lagrangiana efetiva por operadores de dimensão seis. Na prática, o parâmetro U não é muito utilizado, pois contribuições a ele são suprimidas em relação a $S$ e $T$, pois vem de operadores de dimensão oito. Esses parâmetros são calculados para um valor de referência das massas do Higgs e do quark top. Para $m_{t}=173 \mathrm{GeV}$ e $m_{h}=126 \mathrm{GeV}$ o resultado é dado por [76] $S^{\text {exp. }}=0.03 \pm 0.10$ e $T^{\text {exp. }}=0.05 \pm 0.12$, que deve ser comparado com as previsões da física nova.

Integrando fora os modos massivos de gauge, o modelo que consideramos (assim como o modelo de RS) irá gerar operadores que contribuem para $\mathrm{S}$ e $\mathrm{T}$. 
Eles serão parametrizados pela Lagrangiana de dimensão seis [47, 77]:

$$
\begin{aligned}
\mathcal{L}_{6} & \equiv-\frac{g g^{\prime} S_{0}}{8 \pi v_{E W}^{2}} H^{\dagger} T^{a} H B^{\mu \nu} W_{\mu \nu}^{a}-\frac{T_{0} e^{2}}{2 \pi v_{E W}^{2}}\left|\left(D_{\mu} H\right)^{\dagger} H\right|^{2}+ \\
& \left(i \frac{x_{j}}{v_{E W}^{2}} \bar{\psi}_{j} \gamma^{\mu} T^{a} \psi_{j}\left(D_{\mu} H\right)^{\dagger} T^{a} H+i \frac{y_{j} Y_{j}}{2 v_{E W}^{2}} \bar{\psi}_{j} \gamma^{\mu} \psi_{j}\left(D_{\mu} H\right)^{\dagger} H+\frac{V_{i j k l}}{v_{E W}^{2}} \bar{\psi}_{i} \psi_{j} \bar{\psi}_{k} \psi_{l}+\text { h.c. }\right),
\end{aligned}
$$

onde $Y_{j}$ é a hipercarga do férmion $\psi_{j}, W_{\mu \nu}^{a}$ e $B_{\mu \nu}$ são as intensidades de campo de $S U(2)_{L}$ e $U(1)_{Y}$ e $H$ é o dubleto de Higgs. Assumimos que as constantes $S_{0}, T_{0}, x_{j}, y_{j}, V_{i j k l}$ são pequenas e trataremos seus efeitos até $1^{\mathrm{a}}$ ordem. Para entender a modificação induzida por esses operadores, inserimos o valor esperado de vácuo do Higgs $H^{t}=\left(0, v_{E W} / \sqrt{2}\right)$ e a derivada covariante $D_{\mu}=$ $\partial_{\mu}+i g T^{a} W^{a}+i g^{\prime} Y B_{\mu}$. Consideramos primeiro os operadores que não involvem férmions, da primeira linha de (3.113):

$$
\begin{aligned}
& -\frac{T_{0} e^{2}}{2 \pi v_{E W}^{2}}\left|\left(D_{\mu} H\right)^{\dagger} H\right|^{2}=-\frac{T_{0} e^{2} v_{E W}^{2}}{32 \pi}\left(-g W_{3}^{\mu}+g^{\prime} B^{\mu}\right)^{2}=-\frac{T_{0} e^{2} v_{E W}^{2}}{32 \pi} g^{2} g^{\prime 2} Z_{\mu} Z^{\mu}, \\
& -\frac{g g^{\prime} S_{0}}{8 \pi v_{E W}^{2}} H^{\dagger} T^{a} H B^{\mu \nu} W_{\mu \nu}^{a}=-\frac{g g^{\prime} S_{0}}{32 \pi} B_{\mu \nu} W_{3}^{\mu \nu} .
\end{aligned}
$$

Esses operadores contribuem para S e T precisamente com o valor dos parâmetros $S_{0}$ e $T_{0}$ da Lagrangiana. De fato, comparando com (3.107), identificamos a essa ordem:

$$
\begin{gathered}
\Pi_{Z Z}=-\frac{T_{0} e^{2} v_{E W}^{2}}{16 \pi} g^{2} g^{\prime 2} ; \Pi_{W W}=0 \\
\Rightarrow \frac{\Pi_{W W}}{M_{W}^{2}}-\frac{\Pi_{Z Z}}{M_{Z}^{2}}=\frac{T_{0} e^{2}}{4 \pi}=\frac{T e^{2}}{4 \pi}
\end{gathered}
$$


Analogamente, o operador que mistura os termos cinéticos de $B^{\mu}$ e $W_{3}^{\mu}$ contribui $\mathrm{S}=S_{0}$

Passamos a examinar os operadores que involvem correntes fermiônicas em (3.113). As contribuições mais importantes dos operadores de quatro férmions são os vínculos de sabor discutidos na seção anterior, e eles não afetam significativamente os parametros de precisão [47, 77], assim, nos focaremos nos operadores proporcionais a $x_{j}$ e $y_{j}$, que não contribuem diretamente para $\mathrm{S}$ e $\mathrm{T}$, mas modificam as correntes neutra e carregada do MP:

$$
\begin{aligned}
& i \frac{x_{j}}{v_{E W}^{2}} \bar{\psi}_{j} \gamma^{\mu} T^{a} \psi_{j}\left(D_{\mu} H\right)^{\dagger} T^{a} H+\text { h.c }= \\
& \frac{g}{\sqrt{2}} \frac{x_{j}}{4} \bar{\psi}_{j} \gamma^{\mu} T^{ \pm} \psi_{j} W_{\mu}^{ \pm}+\frac{x_{j}}{4}\left(g \psi_{j} W_{3}^{\mu}-g^{\prime} B^{\mu}\right) \bar{\psi}_{j} \gamma_{\mu} T^{3} \psi_{j}, \\
& i \frac{y_{j} Y_{j}}{2 v_{E W}^{2}} \bar{\psi}_{j} \gamma^{\mu} \psi_{j}\left(D_{\mu} H\right)^{\dagger} H+\text { h.c }= \\
& -\frac{y_{j}}{4}\left(g \psi_{j} W_{3}^{\mu}-g^{\prime} B^{\mu}\right) \bar{\psi}_{j} \gamma_{\mu} Y_{j} \psi_{j} .
\end{aligned}
$$

Adicionando a contribuição do MP, vemos que essas contribuições correspondem as modificações nas correntes:

$$
\begin{aligned}
& J_{\mu}^{ \pm} W^{ \pm \mu}=\frac{g}{\sqrt{2}}\left(1+\frac{x_{j}}{4}\right) \bar{\psi}_{j} \gamma^{\mu} T^{ \pm} \psi_{j} W_{\mu}^{ \pm} \\
& J_{Z}^{\mu} Z_{\mu}=\frac{1}{\sqrt{g^{2}+g^{\prime 2}}}\left[g^{2}\left(1+\frac{x_{j}}{4}\left(g^{2}+g^{\prime 2}\right)\right) T_{j}^{3}-g^{\prime 2}\left(1+\frac{y_{j}}{4}\left(g^{2}+g^{\prime 2}\right)\right) Y_{j}\right] \bar{\psi}_{j} \gamma^{\mu} \psi_{j} Z_{\mu} .
\end{aligned}
$$

Vamos supor que $x_{j}$ e $y_{j}$ tenham uma forma universal, independente do férmion ao qual os bósons de gauge se acoplam. Isso será o caso em nosso modelo quando os férmions estiverem localizados no mesmo platô do acoplamento de gauge com 
o primeiro modo massivo (figura (3.4)). Mais precisamente, impomos a condição:

$$
\frac{x_{j}}{g^{2}}=\frac{y_{j}}{g^{\prime 2}}=a \text { para todo } j
$$

Nesse caso, é fácil ver das equações (3.115) e (3.116) que a redefinição dos campos:

$$
\begin{aligned}
& W^{ \pm} \rightarrow W^{ \pm}\left(1-g^{2} \frac{a}{4}\right), \\
& W^{3} \rightarrow W^{3}\left(1-g^{2} \frac{a}{4}\right)+B g g^{\prime} \frac{a}{4}, \\
& B \rightarrow B\left(1-g^{\prime 2} \frac{a}{4}\right)+W^{3} g g^{\prime} \frac{a}{4},
\end{aligned}
$$

restaura as correntes neutra e carregada a sua forma no MP [77]. No entanto, essa mudança gera novas contribuições para S e T. Por exemplo, as massas dos bósons $W$ e $Z$ são modificadas por:

$$
\begin{aligned}
& M_{W}^{2} \rightarrow M_{W}^{2}\left(1-\frac{g^{2} a}{2}\right)=M_{W}^{2}\left(1+\delta \Pi_{W W}\right), \\
& M_{Z}^{2} \rightarrow M_{Z}^{2}\left(1-\frac{a}{2}\left(g^{2}+g^{\prime 2}\right)\right)=M_{Z}^{2}\left(1+\delta \Pi_{Z Z}\right) .
\end{aligned}
$$

Portanto, de (3.112), vemos que T é dado por:

$$
T=T_{0}+\frac{2 \pi g^{2}}{e^{2}} a
$$

Analogamente, analizando a modificação nos tensores de intensidade de campo, vemos que o parâmetro $S$ sofre a mudança:

$$
S=S_{0}+8 \pi a
$$


Vemos que quando as correções de vértice são universais, temos a liberdade de redefini-las como correções oblíquas.

Tendo estabelecido o formalismo, vamos computar S e T para o modelo de cinco sítios, assumindo um dubleto de Higgs localizado no sítio $N$ e o grupo no quiver $S U(2)_{L} \times U(1)_{Y}$. As contribuições para $S$ e T serão geradas pelos modos massivos de gauge. A Lagrangiana de interação com o Higgs é dada, ao substituir o valor esperado de vácuo, por:

$$
\begin{aligned}
\mathcal{L}_{g h}=\frac{1}{2}\left|D_{\mu} H\right|^{2}= & \frac{\tilde{g}^{2} v_{E W}^{2}}{8}\left(\left(W_{1}^{N}\right)^{2}+\left(W_{2}^{N}\right)^{2}+\left(W_{3}^{N}\right)^{2}\right) \\
& +\frac{\tilde{g} \tilde{g}^{\prime} v_{E W}^{2}}{4}\left(W_{3}^{N} B^{N}\right)+\frac{\tilde{g}^{\prime 2} v_{E W}^{2}}{8}\left(B^{N}\right)^{2},
\end{aligned}
$$

onde omitimos os índices de Lorentz. Lembramos que os acoplamentos estão relacionados aos do MP por:

$$
\tilde{g}^{\left({ }^{\prime}\right)}=\sqrt{N+1} g^{\left({ }^{\prime}\right)} .
$$

Expandindo em auto-estados de massa:

$$
W_{\mu}^{a N}=\sum_{j=0}^{N} f_{j, N} W_{\mu}^{a(j)} .
$$

E analogamente para $B_{\mu}^{N}$, vemos que, se tratarmos as inserções do vev do Higgs como uma perturbação, elas induzem mistura entre os modos massivos. Por essa razão, teremos contribuições para $\mathrm{T}$ e $S$ já a nível árvore (o mesmo ocorre em $A d S_{5}$ ). A mistura dos modos zero dos bósons de gauge com os seus modos massivos está representada pelos diagramas das figuras (3.12) e (3.13). 


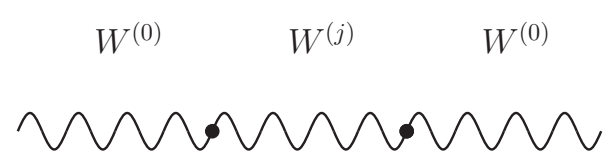

Figura 3.12: Mistura a nível árvore de $W_{a}^{(0)}$ e $W_{a}^{(j)}$.

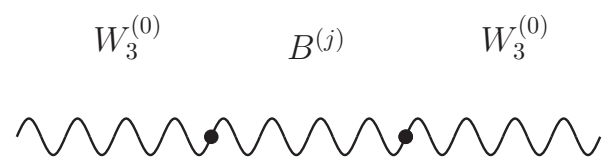

Figura 3.13: Mistura a nível árvore de $W_{3}^{(0)}$ e $B^{(j)}$.

Da Lagrangiana (3.122), vemos que o modo zero de $W_{\mu}^{ \pm}$só se mistura com os seus próprios modos massivos, enquanto que $W_{3}^{\mu}$ se mistura também com os modos massivos de $B^{\mu}$ e vice-versa. Podemos ler o vértice da mistura $W B$ :

$$
i \frac{(N+1) g g^{\prime} v_{E W}^{2}}{4} f_{0, N} f_{j, N}
$$

e da mistura $W W$ :

$$
i \frac{(N+1) g^{2} v_{E W}^{2}}{4} f_{0, N} f_{j, N}
$$

Lembrando que os vevs dos campos de ligação escalam como $v_{j}=v q^{j}$, vemos que, para cinco sítios, o vev do último campo de ligação em $j=4$ será de ordem TeV para $q^{4}=e^{-k a N}=10^{-16}$, o que dá a escala de massas do modo massivo mais leve. O próximo auto-estado terá massa de ordem $v q^{3} \simeq 10^{4} \mathrm{TeV}$, de maneira que suas contribuições podem ser ignoradas com segurança.

Comparando as figuras (3.12) e (3.13) com a figura (3.11), vemos que a ordem mais baixa de teoria de perturbações, as polarizações de vácuo são dadas pelo quadrado desses vértices de mistura vezes o propagador do primeiro modo 
massivo correspondente avaliado para o momento $p^{2}=0$. Assim, temos:

$$
\begin{aligned}
i g^{2} \Pi_{11}(0) & =i \frac{(N+1) g^{2} v_{E W}^{2}}{4} f_{0, N} f_{1, N} \frac{i}{M^{(1) 2}} i \frac{(N+1) g^{2} v_{E W}^{2}}{4} f_{0, N} f_{1, N} \\
& =-i g^{2}\left(\frac{(N+1) g v_{E W}^{2}}{4 M^{(1)}} f_{0, N} f_{1, N}\right)^{2}, \\
i g^{2} \Pi_{33}(0) & =-i g^{2}\left(\frac{(N+1) v_{E W}^{2}}{4} f_{0, N} f_{1, N}\right)^{2}\left(\frac{g^{2}}{M^{(1) 2}}+\frac{g^{\prime 2}}{M^{(1) 2}}\right),
\end{aligned}
$$

onde denotamos a massa do primeiro modo massivo por $M^{(1)}$. A contribuição da mistura $W W$ se cancela entre $\Pi_{11}$ e $\Pi_{33}$, e assim, esses diagramas geram a contribuição para T:

$$
T_{0}=\frac{16 \pi}{v_{E W}^{2} e^{2}}\left[\Pi_{11}(0)-\Pi_{33}(0)\right]=\frac{v_{E W}^{2} \pi}{e^{2}}(N+1)^{2}\left(f_{0, N} f_{1, N}\right)^{2} \frac{g^{\prime 2}}{M^{(1) 2}}
$$

A correspondente contribuição para S dependerá da derivada da polarização do vácuo:

$$
\left.\Pi_{V V}^{\prime}(0) \sim \frac{d}{d p^{2}} \frac{1}{p^{2}-M^{(1) 2}}\right|_{p^{2}=0}=-\frac{1}{M^{(1) 4}}
$$

Que iremos desconsiderar, pois está suprimida por um fator $1 / M^{(1)} 4$. As modificações das correntes fermiônicas são geradas por sua vez por diagramas como os da figura (3.14).

Lembramos que os acoplamentos dos férmions com o primeiro modo massivo em unidades do acoplamento com o modo zero são dados pela equação (3.91):

$$
\frac{g_{01}^{L, R}}{g}=\sqrt{N+1} \sum_{j=0}^{N}\left(Z_{L, R}\right)^{2 j}\left(\frac{1-Z_{L, R}^{2}}{1-Z_{L, R}^{2(N+1)}}\right) f_{1, j}
$$

onde $Z_{L}=q^{c_{L}-1 / 2}$ e $Z_{R}=q^{-\left(c_{R}+1 / 2\right)}$. Quando consideramos a troca de $W_{3}^{(1)}$ 


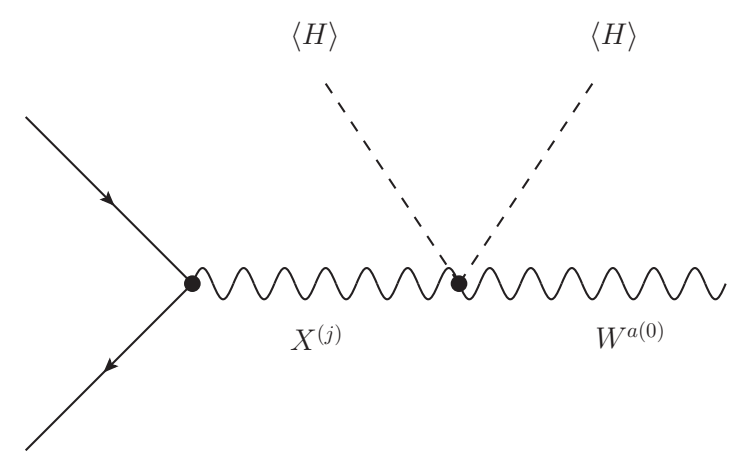

Figura 3.14: Contribuição para a modificação das correntes. $X^{(j)}$ representa qualquer campo que se acople com $W^{a(0)}$.

entre o férmion e o modo zero de $W_{3}$, obtemos o coeficiente proporcional a $x_{j}$ da equação (3.113). Analogamente, a troca de $B^{(1)}$ resulta em uma contribuição para $y_{j}$. Obtemos assim:

$$
\frac{x_{j}}{g^{2}}=\frac{y_{j}}{g^{\prime 2}}=\frac{v_{E W}^{2}}{4}(N+1)^{2} \frac{f_{0, N} f_{1, N}}{M^{(1) 2}} \frac{g_{01 j}}{g} .
$$

No caso em que as localizações são universais, $g_{01 j}=g_{01}$, obtemos então das equações (3.120) e (3.121) uma contribuição para S e T que deve ser somada a já calculada para T (3.126) para chegarmos a expressão final para os parâmetros de precisão eletrofraca:

$$
\begin{aligned}
& T=\frac{v_{E W}^{2} \pi g^{\prime 2}}{e^{2}} \frac{(N+1)^{2}}{M^{(1)} 2} f_{0, N} f_{1, N}\left(f_{0, N} f_{1, N}+\frac{1}{2} \frac{g_{01}}{g}\right), \\
& S=2 \pi v_{E W}^{2}(N+1)^{2} \frac{f_{0, N} f_{1, N}}{M^{(1) 2}} \frac{g_{01}}{g} .
\end{aligned}
$$

Para avaliar S e T, precisamos saber o valor de $f_{0, N}, f_{1, N}$ e $g_{01}$. Como a função 
de onda do modo zero é plana, temos simplesmente $f_{0, N}=1 / \sqrt{N+1}=1 / \sqrt{5}$ da equação (3.28). Para férmions localizados nos platôs, podemos ler o valor de $g_{01} / g$ do gráfico do acoplamento para $N=4$, mostrado na figura (3.4). Temos os valores $g_{01} /\left.g\right|_{U V} \simeq-0.5$ e $g_{01} /\left.g\right|_{I R} \simeq 2$. Podemos relacionar esses valores a $f_{1, N}$ se notamos que, para férmions localizados em $j=0$ :

$$
\left.\frac{g_{01}^{L, R}}{g}\right|_{U V}=\lim _{Z_{L, R} \rightarrow 0} \sqrt{N+1} \sum_{j=0}^{N}\left(Z_{L, R}\right)^{2 j}\left(\frac{1-Z_{L, R}^{2}}{1-Z_{L, R}^{2(N+1)}}\right) f_{1, j}=\sqrt{N+1} f_{1,0} .
$$

Similarmente, para férmions no sítio $\mathrm{N}$ :

$$
\left.\frac{g_{01}^{L, R}}{g}\right|_{I R}=\lim _{Z_{L, R} \rightarrow \infty} \sqrt{N+1} \sum_{j=0}^{N}\left(Z_{L, R}\right)^{2 j}\left(\frac{1-Z_{L, R}^{2}}{1-Z_{L, R}^{2(N+1)}}\right) f_{1, j}=-\sqrt{N+1} f_{1, N} .
$$

Ou seja, $f_{1, N} \simeq-2 / \sqrt{5}$. Para calcular $\mathrm{S}$ e $\mathrm{T}$, precisamos definir a parte universal das correções de vértice que será atríbuida às correções oblíquas (naturalmente, isso é uma convenção, e o ajuste da teoria deve levar em conta também as correções não-oblíquas). Escolhemos tomar a localização do platô de mão esquerda como a parte universal nos casos A e B. Como no caso A os dubletos estão localizados no sítio $j=N$, e no caso B no sítio $j=0$ (exceto a terceira geração), as contribuições fermiônicas para S e T irão diferir entre os dois casos pela razão $\left.g_{01}^{L}\right|_{I R} /\left.g_{01}^{L}\right|_{U V} \simeq-4$. O resultado obtido para o caso B é:

$$
\begin{aligned}
& S \simeq 0.17 \times\left(\frac{3 \mathrm{TeV}}{M^{(1)}}\right)^{2} \\
& T \simeq 0.16 \times\left(\frac{3 \mathrm{TeV}}{M^{(1)}}\right)^{2},
\end{aligned}
$$

que deve ser comparado com o valor do ajuste experimental para $m_{t}=173 \mathrm{GeV}$ 
e $m_{h}=126 \mathrm{GeV}$, dado por [76] $S^{\text {exp. }}=0.03 \pm 0.10$ e $T^{\text {exp. }}=0.05 \pm 0.12$. Vemos então que a massa $M^{(1)}=3 \mathrm{TeV}$ está dentro do intervalo de confiança de $95 \%$. Este valor é o bastante para satisfazer os vínculos de precisão e também os de sabor, como vimos. Em contraste, um cálculo semelhante feito para o modelo de RS resulta em contribuições muito grandes para o parâmetro T, que em geral requerem a extensão do grupo de gauge no bulk para $S U(2)_{L} \times S U(2)_{R} \times U(1)_{X}$, que é quebrado ao grupo eletrofraco do MP por condições de contorno [77, 47, 48]. Contribuições adicionais para $S$ e $T$ virão de loops envolvendo também os modos massivos dos férmions, e são importantes especialmente para a terceira geração. Estas correções, bem como contribuições vindas dos campos de ligação deverão ser estudadas no futuro.

Iremos agora extender nosso modelo para um caso mais realista, onde o Higgs é um pseudo bóson de Nambu-Goldstone, e se localiza naturalmente próximo ao sítio infravermelho. 


\section{Capítulo 4}

\section{O Higgs Como um Pseudo Bóson de Nambu-Goldstone}

\subsection{Motivação}

De maneira geral, como argumentamos no capítulo (1), um desafio enfrentado por modelos de física além do MP que buscam resolver o problema da hierarquia de gauge é o fato de que os vínculos de sabor e de precisão eletrofraca requerem que a escala de física nova $M_{B S M}$ seja elevada a vários $\mathrm{TeV}$, enquanto que a escala eletrofraca é de apenas $M_{E W} \sim 100 \mathrm{GeV}$. De fato, o recém descoberto candidato a bóson de Higgs [6, 7] possui massa $M_{h} \simeq 125 \mathrm{GeV}$ (um valor leve, que já era favorecido pelos ajustes dos parâmetros de precisão [3]), enquanto que ainda não há evidências da existência de outras partículas preditas por extensões do MP, o que indica que, caso existam, suas massas deve estar perto ou acima da escala TeV. Assim, mesmo para modelos que apresentam soluções naturais do problema da hierarquia de gauge, muitas vezes ainda é necessário um pequeno 
ajuste fino de ordem $M_{E W} / M_{B S M}$, que podemos chamar de um "pequeno problema da hierarquia" $[78,79,80,81]$.

Uma maneira de garantir que a separação entre as escalas ocorra naturalmente, é fazer com que a massa do Higgs seja protegida por uma simetria. Conhecemos uma maneira natural de obter escalares leves na teoria quântica de campos: o mecanismo de Goldstone. De fato, bósons de NG são escalares naturalmente sem massa mesmo a nível quântico, pois sua massa é protegida por uma simetria global. Na natureza encontramos exemplos de partículas escalares cuja massa leve é explicada por corresponderem a bósons de NG. Os píons na QCD podem ser vistos como bósons de NG da quebra espontânea da simetria quiral, o que garante a separação de escalas entre a massa do píon e a dos outros hádrons [8]. Como a simetria quiral é quebrada explicitamente pelas massas dos quarks up e down e pela interação eletromagnética, os píons não são bósons de NG exatos e adquirem uma pequena massa. Por essa razão, eles são chamados de pseudo bósons de Nambu-Goldstone. Nesta seção, veremos como o pequeno problema da hierarquia se manifesta em modelos de dimensões extras curvas, e uma possível solução, depois discutiremos a versão desconstruída desse mecanismo e como ela se insere no contexto mais amplo do Higgs como um PBNG.

Para modelos em $A d S_{5}$, vimos que é possível gerar naturalmente uma hierarquia de escalas através da geometria da dimensão extra curva. Assim, desde que o bóson de Higgs esteja localizado próximo a brana IR, temos uma solução para o problema da hierarquia de gauge. No entanto, a solução não é completamente satisfatória, pois não consideramos a razão dinâmica da localização do Higgs. Além disso, como vimos, os vínculos de precisão eletrofraca e de sabor exigem que a escala de massas dos modos de KK esteja em torno de $M_{K K} \sim 10 \mathrm{TeV}[57,48,77]$, 
enquanto que a escala eletrofraca é de ordem $M_{E W} \sim 100 \mathrm{GeV}$. Se tentamos explicar a origem dessas duas escalas através do fator de warping $M_{I R}=M_{U V} e^{-k \pi R}$, vemos que ainda é necessário um ajuste fino de ordem $M_{E W} / M_{K K} \sim 10^{-3}$.

Tanto o pequeno problema da hierarquia quanto o problema da localização do Higgs podem ser resolvidos em modelos de WED se considerarmos os graus de liberdade que constituem o dubleto do Higgs como parte do campo de gauge que se propaga no bulk cinco dimensional, na chamada unificação gauge-Higgs (Gauge-Higgs Unification - GHU) [82, 83, 84, 85]. Não iremos desenvolver esse tema em detalhe aqui, mas apenas apontar as características principais desse tipo de modelo, para tornar mais intuitivo o que faremos na versão desconstruída nas próximas sessões.

A primeira vista, pode parecer impossível obter o Higgs, que é um escalar na representação fundamental de $S U(2)_{L}$, de um campo de gauge, que é um vetor e vive na representação adjunta. A questão do spin é resolvida se lembramos que o campo de gauge cinco dimensional se decompõe como $A_{M}=\left(A_{\mu}, A_{5}\right)$, onde sob o ponto de vista quadridimensional, $A_{\mu}$ é um vetor e $A_{5}$ é um escalar. De fato, vimos na descrição de modos de KK do campo de gauge (seção (2.2)) que as componentes $A_{5}$ podém ser vistas como bósons de NG da quebra da simetria translacional no orbifold, que são absorvidos e dão massa para os modos de KK. Assim, poderemos obter o Higgs se o espectro contiver modos zero de $A_{5}$, que são escalares leves em quatro dimensões. Já o problema da representação é resolvido estendendo o grupo de gauge no bulk a um grupo maior, que contém o grupo eletrofraco como subgrupo. Esse grupo maior é então quebrado por condições de contorno no orbifold, de maneira tal que as componentes $A_{5}$ correspondentes a um dubleto de $S U(2)_{L}$ obtenham um modo zero. 
A escolha mais simples de grupo no bulké $S U(3)$. De fato, na quebra $S U(3) \rightarrow$ $S U(2) \times U(1)$, existem quatro bósons de NG, que é o número de graus de liberdade necessários para o dubleto complexo de $S U(2) .{ }^{1}$ Expandimos o octeto de $S U(3)$ segundo $A_{M}=A_{M}^{A} T^{A}$, e tomamos a base de geradores do grupo $S U(3)$ como $T^{A}=\lambda^{A} / 2$, em que $\lambda^{A}$ são as matrizes de Gell-Mann:

$$
\begin{aligned}
& \lambda^{1}=\left(\begin{array}{lll}
0 & 1 & 0 \\
1 & 0 & 0 \\
0 & 0 & 0
\end{array}\right), \quad \lambda^{2}=\left(\begin{array}{rrr}
0 & -i & 0 \\
i & 0 & 0 \\
0 & 0 & 0
\end{array}\right), \quad \lambda^{3}=\left(\begin{array}{rrr}
1 & 0 & 0 \\
0 & -1 & 0 \\
0 & 0 & 0
\end{array}\right) \\
& \lambda^{4}=\left(\begin{array}{lll}
0 & 0 & 1 \\
0 & 0 & 0 \\
1 & 0 & 0
\end{array}\right), \quad \lambda^{5}=\left(\begin{array}{ccc}
0 & 0 & -i \\
0 & 0 & 0 \\
i & 0 & 0
\end{array}\right), \quad \lambda^{6}=\left(\begin{array}{lll}
0 & 0 & 0 \\
0 & 0 & 1 \\
0 & 1 & 0
\end{array}\right), \\
& \lambda^{7}=\left(\begin{array}{ccc}
0 & 0 & 0 \\
0 & 0 & -i \\
0 & i & 0
\end{array}\right), \lambda^{8}=\frac{1}{\sqrt{3}}\left(\begin{array}{ccc}
1 & 0 & 0 \\
0 & 1 & 0 \\
0 & 0 & -2
\end{array}\right) \text {. }
\end{aligned}
$$

Nessa base, uma escolha simples de orientação dos geradores do subgrupo $S U(2) \times U(1)$ consiste em tomar o subgrupo $S U(2)$ gerado pelos $\tau^{i} \equiv \lambda^{i} / 2, i=$ 1, 2, 3, e o gerador de $U(1)$ como $T^{8}$ (com uma possível normalização). Os outros quatro geradores se transformam sob o subgrupo $S U(2)$ como um dubleto complexo (quatro graus de liberdade), o que é garantido pela decomposição da adjunta de $S U(3)$ no subgrupo $S U(2): \mathbf{8} \rightarrow \mathbf{3} \oplus \mathbf{2} \oplus \overline{\mathbf{2}} \oplus \mathbf{1}$. Assim, escolhemos as condições de contorno do octeto de gauge de $S U(3)$ no orbifold segundo:

$$
A_{\mu}=\left(\begin{array}{ccc}
(++) & (++) & (--) \\
(++) & (++) & (--) \\
(--) & (--) & (++)
\end{array}\right), \quad A_{5}=\left(\begin{array}{ccc}
(--) & (--) & (++) \\
(--) & (--) & (++) \\
(++) & (++) & (--)
\end{array}\right)
$$

\footnotetext{
${ }^{1}$ Outra possibilidade é a quebra $S O(5) \rightarrow S O(4)$, onde $S O(4) \cong S U(2)_{L} \times S U(2)_{R}$ contém o grupo custodial $S U(2)_{R}$. A hipercarga é então tomada como o gerador $T_{R}^{3}$.
} 
onde $(++)$ se refere a condições de contorno de Neumann nas branas UV e IR, respectivamente, (o que garante a existência de um modo zero) enquanto que (--) se refere a condições de Dirichlet (nesse caso, não há modo zero). Lembramos que para preservar a simetria $\mathbb{Z}_{2}$, quando $A_{\mu}$ obedece condições de contorno $(++), A_{5}$ tem de ser $(--)$ e vice versa. Vemos que sob essas condições de contorno, o espectro de baixa energia (modos zero) conterá os bósons de gauge de $S U(2) \times U(1),\left(A_{\mu}^{1}, A_{\mu}^{2}, A_{\mu}^{3}, A_{\mu}^{8}\right)$, mais um dubleto escalar $\left(A_{5}^{4}+i A_{5}^{5}, A_{5}^{6}+i A_{5}^{7}\right)$, que vamos identificar com o Higgs.

Usando as equações de movimento e a expansão em modos de KK de $A_{5}$, de maneira análoga ao que fizemos na seção (2.2), pode-se mostrar que o modo zero de $A_{5}^{A}$ é localizado segundo $A_{5}^{(0), A}(x, y)=h(x)^{A} e^{k y}$, ou seja, localizado no infravermelho, como precisamos para resolver o problema da hierarquia de gauge $[86,43,44]$.

A nível árvore, os modos zero tem massa nula, mas obviamente se quisermos efetuar a EWSB precisamos de um potencial não trivial para o Higgs. A questão mais importante a resolver nesse tipo de modelo é então como gerar uma massa e um termo quártico para os modos zero de $A_{5}$. Como $A_{5}$ é parte de um campo de gauge, esperamos que sua massa seja protegida pela simetria de gauge. Porém, surpreendentemente, é possível adquirir uma massa para $A_{5}$ por correções radiativas devido a geometria não trivial da dimensão extra. Podemos considerar a linha de Wilson, $W \equiv P \exp \left(i \int d y A_{5}\right)$, que se enrola em torno do círculo $S_{1}$ em que a dimensão extra é compactificada. Esse loop de Wilson não pode ser contraído a um ponto, e o valor da fase de Aharonov-Bohm adquirida ao dar uma volta em torno da dimensão extra se torna uma variável dinâmica. O valor da fase é afetado por correções radiativas, e determina o potencial de $A_{5}[43,87,44]$. 
De fato, se gerarmos o operador $|\operatorname{Tr}[W]|^{2}$, que é invariante de gauge, vemos que ele contém uma massa para o modo zero de $A_{5}$ em sua expansão. Além disso, como esse operador é não local na dimensão extra, logo, não pode haver uma divergência ultravioleta associada a ele, o que garante que o potencial efetivo é finito $[87,86]$. Tanto os acoplamentos de gauge quanto os de Yukawa geram um potencial a 1-loop para o Higgs, que pode ser calculado utilizando o formalismo de Coleman-Weinberg [88]. Naturalmente, a massa adquirida por esse mecanismo será da ordem da escala de compactificação, $\sim M_{K K}$, dividida por um fator de loop, o que garante a separação das escalas $M_{K K}$ e $m_{h}$ (pequena hierarquia) $[87,86,43]$.

Estamos interessados na versão desconstruída desse mecanismo, que nos dará um Higgs naturalmente leve e localizado no sítio $N$. Essa questão foi analisada para dimensões extras planas compatificadas em um círculo em [87]. A versão desconstruída é então uma cadeia circular (identificamos os sítios 0 e $N$ ) em que todos os campos de ligacão adquirem um vev comum (geometria plana). Como a linha de Wilson corresponde ao campo de ligação segundo nosso dicionário (3.46), o Higgs será obtido como parte dos $\pi_{j}$, ou seja, ele será um bóson de NG. O loop de Wilson que dá a volta na dimensão extra corresponde na versão desconstruída ao operador $\operatorname{Tr}\left[\Phi_{1} \Phi_{n} \ldots \Phi_{N}\right]$, que não pode ser removido por transformações de gauge. Expandindo esse operador, vemos que o espectro da teoria contém o bóson $h \equiv 1 / \sqrt{N}\left(\pi_{1}+\pi_{2}+\ldots \pi_{N}\right)$, que não é absorvido. Uma vez que as interações de gauge quebram explicitamente a simetria global, esse estado recebe um potencial não trivial por correções radiativas e é identificado com o Higgs. De fato, pode-se mostrar que o potencial é livre de divergências quadráticas para $N>1$ e finito para três sítios ou mais [87]. 
A razão da ausência de divergências quadráticas para o potencial independe da origem extra dimensional deste tipo de modelo. Em última análise, ela vem da estrutura da quebra de simetria no quiver. A contribuição de diagramas de bósons de gauge de qualquer um dos sítios individuais para a massa do Higgs seria quadraticamente divergente, no entanto, como os operadores gerados para o Higgs envolvem toda a cadeia (são não lineares no quiver), $\mathcal{O} \sim\left|\operatorname{Tr}\left[\Phi_{1} \Phi_{n} \ldots \Phi_{N}\right]\right|^{2}$ [87], a contribuição para o potencial é proporcional ao produto dos acoplamentos de gauge $g_{1} g_{2} \ldots g_{N}$. Se qualquer um dos acoplamentos for zero, a contribuicão é nula, e por essa razão, uma divergência quadrática não pode ser gerada, o que é chamado de mecanismo de quebra de simetria coletiva. Uma grande variedade de modelos em que Higgs é um PBNG cuja massa é protegida de divergências quadráticas pela quebra coletiva pode ser construída, e em sua grande maioria eles não tem uma interpretacão como dimensão extra. Tais modelos são chamados de modelos de Little Higgs (review [89]) e são uma área muito ativa de pesquisa.

Apresentaremos na sessão seguinte um modelo desconstruído de uma dimensão extra curva que contém no espectro um PBNG localizado naturalmente no sítio N, que identificaremos com o bóson de Higgs. Também mostraremos nossos resultados preliminares para o seu potencial que é gerado por correções radiativas e finito a 1-loop para modelos de três ou mais sítios.

\subsection{O Modelo}

Para obter um modelo desconstruído onde o Higgs é um PBNG, precisamos estudar uma cadeia linear um pouco mais complexa do que no capítulo anterior. 
Em geral os bósons de NG parametrizam o espaço $G / H$ da quebra espontânea da simetria global $G \rightarrow H$, onde $H$ é um subgrupo de $G$. No caso em que existem bósons de gauge associados aos geradores de $G / H$, os bósons de NG são absorvidos e se tornam as suas componentes longitudinais, pelo mecanismo de Higgs. No entanto, se não houver um campo de gauge correspondente, os modos de NG permanecerão no espectro. Em outras palavras, precisamos que a simetria de gauge seja apenas um subgrupo da simetria global da teoria, para que alguns dos bósons de NG sejam físicos. Além disso, a simetria de gauge (assim como os acoplamentos de Yukawa com férmions) deve quebrar explicitamente a simetria global, para que os bósons de NG adquiram um potencial efetivo não trivial por correções radiativas, como veremos.

O grupo de simetria global deve conter o grupo eletrofraco $S U(2) \times U(1)$ como subgrupo. Como vimos na sessão anterior, a escolha mais simples é $S U(3)$. Ligando as interações de gauge $S U(2)_{L} \times U(1)_{Y}$, a simetria global é explicitamente quebrada pelos acoplamentos de gauge $g$ e $g^{\prime}$, permitindo gerar um potencial a 1-loop. Baseados nessas considerações, tomamos a cadeia linear com simetria global $S U(3)_{j-1} \times S U(3)_{j}$ para cada campo de ligação. Assim, os $\Phi_{j}$ são responsáveis pela quebra $S U(3)_{j-1} \times S U(3)_{j} \rightarrow S U(3)_{d i a g .,}$ e portanto contêm um octeto de bósons de NG $\pi_{j}^{A} T^{A}$, onde $T^{A}$ são os geradores de $S U(3)$.

Tomaremos como simetria de gauge nos sítios zero e $N$ apenas o subgrupo eletrofraco $S U(2) \times U(1)$, enquanto que nos sítios internos tornamos local todo o grupo $S U(3)$. Como os sítios nas extremidades não contém bósons de gauge associados aos geradores de $S U(3) / S U(2) \times U(1)$, restarão alguns bósons de NG que não poderão ser absorvidos, correspondentes aos quatro graus de liberdade do dubleto de Higgs. Além disso, como tornamos local apenas um subgrupo 
$S U(2) \times U(1)$ nas extremidades do quiver, quebramos explicitamente a simetria global, o que permitirá gerar o potencial efetivo. Essa maneira de obter o Higgs a partir dos campos de ligação é análoga a descrita em [87] para uma cadeia circular, mas, no caso aqui apresentado, temos uma hierarquia de vevs que gerará dinamicamente a localização do bóson de Higgs próximo ao sítio $N$. O diagrama quiver correspondente a esse modelo está exibido na figura (4.1). No limite do contínuo, essa cadeia corresponde a uma simetria $S U(3)$ no bulk quebrada a $S U(2) \times U(1)$ nas branas, em analogia com o modelo de GHU da seção anterior.

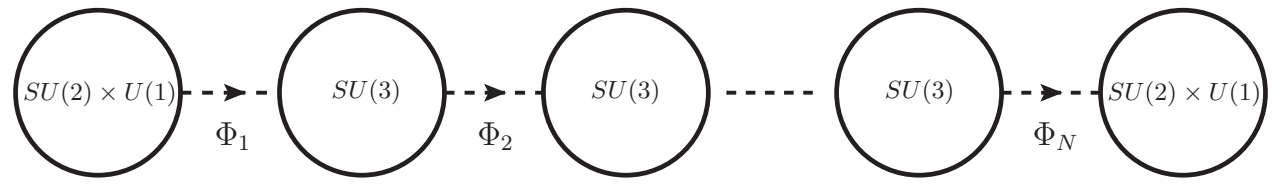

Figura 4.1: Diagrama quiver para uma cadeia de grupos de gauge. Os grupos das extremidades, sítios 0 e $N$, são $S U(2) \times U(1)$ e os demais são $S U(3)$. A simetria global, não representada, é $S U(3)$ em todos os sítios.

Como na seção anterior, tomamos a base de geradores dos grupos $S U(3)$ como $T^{A}=\lambda^{A} / 2$, em que $\lambda^{A}$ são as matrizes de Gell-Mann (4.1). Denotaremos o conjunto de geradores de $S U(2) \times U(1)$ por $Y^{a} \equiv\left\{T^{1}, T^{2}, T^{3}, T^{8}\right\}$, enquanto que os outros quatro geradores serão denotados por $X^{\alpha} \equiv\left\{T^{4}, T^{5}, T^{6}, T^{7}\right\}$. Convencionamos que os índices latinos $a, b, c, \ldots$ assumem os valores $1,2,3,8$ e os indíces gregos $\alpha, \beta, \gamma, \ldots$ os valores $4,5,6,7$. Por sua vez os índices capitalizados $A, B, C, \ldots$ irão de um a oito. As constantes de estrutura de $S U(3)$ não nulas são:

$$
\begin{aligned}
& f_{123}=1, \\
& f_{147}=f_{165}=f_{246}=f_{257}=f_{345}=f_{376}=\frac{1}{2}, \\
& f_{458}=f_{678}=\frac{\sqrt{3}}{2},
\end{aligned}
$$


mais as que podem ser obtidas dessas por uma permutação dos índices. Assim, observamos que a álgebra de $S U(3)$ se decompõe em:

$$
\begin{aligned}
& {\left[X^{\alpha}, X^{\beta}\right]=i f_{\alpha \beta c} Y^{c},} \\
& {\left[Y^{a}, X^{\beta}\right]=i f_{a \beta \gamma} X^{\gamma},} \\
& {\left[Y^{a}, Y^{b}\right]=i f_{a b c} Y^{c} .}
\end{aligned}
$$

Denotamos os acoplamentos dos grupos $S U(2)$ e $U(1)$ dos sítios $j=0$ e $j=N$ por $g_{0, N}$ e $g_{0, N}^{\prime}$, respectivamente. O acoplamento de gauge dos sítios internos será tomado independente de $j$, por simplicidade, e denotado como $\tilde{g}$. Lembramos que para satisfazer as condições de identificação com o contínuo (equações (3.46)), devemos ter o valor esperado de vácuo $v=1 / g a$, onde $g$ é o acoplamento de gauge. Estritamente, isso requer um conjunto de campos de ligação para cada grupo de gauge no quiver e leva a um espectro de modos massivos independente das constantes de acoplamento. Neste capítulo optamos por tomar $v=1 / a$ independente dos acoplamentos. Nesse caso, eliminamos a degenerecência entre os modos associados aos geradores de $S U(2)$ e aos de $U(1)$.

Para obter o espectro de bósons de gauge e de NG a nível árvore, prosseguimos como na seção (3.2). Expandindo o termo cinético dos $\Phi_{j}$, até segunda ordem em $\pi_{j}$, obtemos a mistura entre os bósons de NG e os de gauge. Para os campos associados aos geradores $Y^{a}$, a mistura é dada por:

$$
\sum_{j=1}^{N} \frac{1}{2}\left[\partial_{\mu} \pi_{j}^{a}-v_{j}\left(g_{j} A_{\mu, j}^{a}-g_{j-1} A_{\mu, j-1}^{a}\right)\right]^{2} .
$$


Ou seja, existe um modo de NG para cada bóson de gauge. Assim, todos os bósons de NG associados aos $Y^{a}$ são removidos no gauge unitário exatamente como na seção (3.2), levando a matriz de massas do bósons de gauge de $S U(2)=$ $Y^{1,2,3}$ :

$M_{W}^{2}=v^{2}\left(\begin{array}{cccccc}g_{0}^{2} q^{2} & -g_{0} \tilde{g} q^{2} & 0 & \cdots & 0 & 0 \\ -g_{0} \tilde{g} q^{2} & \tilde{g}^{2}\left(q^{2}+q^{4}\right) & -\tilde{g}^{2} q^{4} & \cdots & 0 & 0 \\ 0 & -\tilde{g}^{2} q^{4} & \tilde{g}^{2}\left(q^{4}+q^{6}\right) & \cdots & 0 & 0 \\ \vdots & \vdots & \vdots & \ddots & \vdots & \vdots \\ 0 & 0 & 0 & \cdots & \tilde{g}^{2}\left(q^{2(N-1)}+q^{2 N}\right) & -g_{N} \tilde{g} q^{2 N} \\ 0 & 0 & 0 & \cdots & -g_{N} \tilde{g} q^{2 N} & g_{N}^{2} q^{2 N}\end{array}\right)$.

Para $U(1)=Y^{8}$, basta substituir $g_{0, N} \rightarrow g_{0, N}^{\prime}$. Como resta uma simetria $S U(2) \times U(1)$ não quebrada, o determinante dessa matriz de massas é nulo, e temos um modo zero, cuja função de onda $f_{j, 0}$ satisfaz:

$$
\begin{aligned}
& g_{0} f_{0,0}=\tilde{g} f_{1,0}, \\
& \tilde{g}\left(q^{2}+q^{4}\right) f_{1,0}=g_{0} q^{2} f_{0,0}+\tilde{g} q^{4} f_{2,0}, \\
& \vdots \\
& \tilde{g} f_{N-1,0}=g_{N} f_{N, 0} .
\end{aligned}
$$

Definindo $\tilde{f}_{j, 0} \equiv g_{j} f_{j, 0}$, temos a relação de recorrência $\tilde{f}_{j, 0}=\tilde{f}_{j-1,0}, \forall j$ como na equação (3.26). Assim, impondo a condição de normalização $\sum_{j=0}^{N}\left|f_{j, 0}\right|^{2}=1$, temos a solução:

$$
f_{j, 0}=\frac{g_{\text {eff }}}{g_{j}},
$$


onde definimos o acoplamento efetivo do grupo diagonal:

$$
\frac{1}{g_{\text {eff }}^{2}} \equiv \sum_{j=0}^{N} \frac{1}{g_{j}^{2}}=\frac{1}{g_{0}^{2}}+\frac{N-1}{\tilde{g}^{2}}+\frac{1}{g_{N}^{2}} .
$$

Vemos que o modo zero não é mais plano, sofrendo uma pequena deformação nos sítios $j=0$ e $j=N$. Vamos estudar o efeito dessa modificação no acoplamento do modo zero a férmions. Considerando um férmion de mão esquerda no quiver, o acoplamento deste ao bóson de gauge é dado por:

$$
\mathcal{L}_{g}=\sum_{j=0}^{N} g_{j} \bar{\psi}_{L, j} \gamma^{\mu} A_{\mu, j} \psi_{L, j}
$$

onde $A_{\mu, j}=A_{\mu, j}^{a} Y_{j}^{a}, \operatorname{com} Y_{j}^{a}$ o gerador na representação do férmion $\psi_{L, j}$. O acoplamento entre os modos zero é obtido fazendo as expansões em modos massivos $\psi_{L, j}=h_{j, m}^{L} \psi_{L, m}^{\prime}$ e $A_{\mu, j}=f_{j, n} A_{\mu, n}^{\prime}$ e tomando $m=n=0$. Para o grupo $S U(2)_{L}$, vamos assumir que o gerador diagonal na representação do férmion é dado simplesmente por $\tau^{a}=\sum_{j=0}^{N} Y_{j}^{a}$. Nesse caso, obtemos:

$$
g_{00}=\sum_{j=0}^{N} g_{j} f_{j, 0}\left|h_{j, 0}^{L}\right|^{2}=g_{e f f},
$$

onde substituimos (4.8) e utilizamos a normalização $\sum_{j=0}^{N}\left|h_{j, 0}^{L}\right|^{2}=1$. Vemos que podemos identificar $g_{\text {eff }}$ com o acoplamento de $S U(2)_{L}$ do MP, $g$. Para a hipercarga, veremos mais adiante que essa escolha simples não será possível se quisermos obter a relação a nível árvore $M_{W}^{2} / M_{Z}^{2}=c_{w}^{2} \simeq 0.8$. De fato, em modelos de GHU, obter o angulo de Weinberg correto requer estender a simetria de gauge no bulk para $S U(3) \times U(1)$ ou adicionar termos cinéticos com acoplamentos diferen- 
tes nas branas, que são permitidos pelo orbifold [90]. ${ }^{2}$ Nossa cadeia corresponde ao segundo caso, já que deixamos livres os acoplamentos nos sítios $j=0, N$.

Para os geradores $X^{\alpha}$, os termos de mistura com os $\pi_{j}^{\alpha}$ são dados por:

$$
\begin{aligned}
& \left.\frac{1}{2}\left[\partial_{\mu} \pi_{1}^{\alpha}-v_{1} \tilde{g} A_{\mu, 1}^{\alpha}\right)\right]^{2}+\sum_{j=2}^{N-1} \frac{1}{2}\left[\partial_{\mu} \pi_{j}^{\alpha}-v_{j} \tilde{g}\left(A_{\mu, j}^{\alpha}-A_{\mu, j-1}^{\alpha}\right)\right]^{2} \\
& \left.+\frac{1}{2}\left[\partial_{\mu} \pi_{N}^{\alpha}+v_{N} \tilde{g} A_{\mu, N-1}^{a}\right)\right]^{2} .
\end{aligned}
$$

Notamos a ausência das misturas $\sim \pi_{1} A_{0} \mathrm{e} \sim \pi_{N} A_{N}$, devido a falta dos geradores correspondentes nas extremidades da cadeia. Introduzimos o termo de fixação de gauge:

$$
\mathcal{L}_{G}=-\sum_{j=1}^{N-1} \frac{1}{2 \xi}\left[\partial_{\mu} A_{j}^{\mu, \alpha}+\xi \tilde{g}\left(v_{j} \pi_{j}^{\alpha}-v_{j+1} \pi_{j+1}^{\alpha}\right)\right]^{2} .
$$

Como anteriormente, as misturas são canceladas, por construção. No entanto, as matrizes de massa são diferentes. Para os bósons de gauge, temos:

$$
M_{A}^{2}=\tilde{g}^{2} v^{2}\left(\begin{array}{ccccc}
q^{2}+q^{4} & -q^{4} & 0 & \cdots & 0 \\
-q^{4} & q^{4}+q^{6} & -q^{6} & \ldots & 0 \\
\vdots & \vdots & \vdots & \cdots & \vdots \\
0 & 0 & 0 & -q^{2 N} & q^{2(N-1)}+q^{2 N}
\end{array}\right) .
$$

Comparando essa matriz com o caso anterior, (4.6) vemos que faltam os termos correspondentes aos bósons das extremidades. Isso é equivalente a impor o aná-

\footnotetext{
${ }^{2}$ Um problema semelhante ocorre em teorias de grande unificação. Por exemplo, para $S U(5)$, $s_{w}^{2}=3 / 8$. Nesse caso, o problema é resolvido notando que os acoplamentos sofrem uma correção grande devida a evolução sob o grupo de renormalização desde $M_{G U T}=10^{16} \mathrm{GeV}$ até a escala eletrofraca.
} 
logo a condições de contorno de Dirichlet no quiver para as funções de onda, $f_{0, j}^{\alpha}=f_{N, j}^{\alpha}=0$, eliminando o modo zero dos $A_{\mu}^{\alpha} X^{\alpha}$. De fato, pode-se checar explicitamente que $\operatorname{Det}\left[M_{A}^{2}\right] \neq 0$. Assim, o espectro dos bósons de gauge associados aos geradores de $S U(3) / S U(2) \times U(1)$ corresponde a uma torre de $N-1$ modos massivos.

A matriz de massas dos bósons de NG por sua vez, será dada por:

$$
M_{\pi}^{2}=g^{2} v^{2} \xi\left(\begin{array}{cccccc}
q^{2} & -q^{3} & 0 & \cdots & 0 & 0 \\
-q^{3} & 2 q^{4} & -q^{5} & \cdots & 0 & 0 \\
0 & -q^{5} & 2 q^{6} & \cdots & 0 & 0 \\
\vdots & \vdots & \vdots & \ddots & \vdots & \vdots \\
0 & 0 & 0 & \cdots & 2 q^{2(N-1)} & -q^{2 N-1} \\
0 & 0 & 0 & \cdots & -q^{2 N-1} & q^{2 N}
\end{array}\right) .
$$

Nesse caso, $\operatorname{Det}\left[M_{\pi}^{2}\right]=0$, o que indica a presença de um modo zero, como esperávamos da estrutura da cadeia. Esse modo é um estado físico, que não se desacopla no limite do gauge unitário, $\xi \rightarrow \infty$. Os outros $N-1$ modos massivos associados aos $X^{\alpha}$ estão em correspondência um a um com os modos de gauge, e são absorvidos no gauge unitário, como requerido do mecanismo de Higgs.

Queremos associar o modo zero da matriz dos bósons de NG ao dubleto do bóson de Higgs. Expandindo $H^{\alpha}=h_{j} \pi_{j}^{\alpha}$, vemos da equação de autovalores $M_{\pi}^{2} \cdot H=0 . H$, que os $h_{j}$ satisfazem a relação de recorrência:

$$
h_{j}=q h_{j+1}
$$

Como $0<q<1$, vemos que o estado $H^{\alpha}=\sum_{j=1}^{N} h_{j} \pi_{j}^{\alpha}$ é um estado localizado em $j=N$, exatamente como esperamos para resolver o problema da hierarquia de 
gauge. Impondo a normalização $\sum_{j=1}^{N}\left|h_{j}\right|^{2}=1$, chegamos a expressão:

$$
H^{\alpha}=\sum_{j=1}^{N} \frac{q^{N-j}}{\sqrt{\sum_{j=1}^{N} q^{2(j-1)}}} \pi_{j}^{\alpha} .
$$

Podemos agora fixar o acoplamento do modo zero do grupo $U(1)$ diagonal, $g_{e f f}^{\prime}$, se expandirmos a derivada covariante $D_{\mu} \Phi_{j}$ até a ordem mais baixa em $\pi_{j}$ e substituirmos as expressões dos modos zero (4.8) e (4.17):

$$
\begin{aligned}
& D_{\mu} \frac{\Phi_{j}}{v_{j}}=\partial_{\mu} \pi_{j}+i g_{j-1} B_{\mu, j-1} \pi_{j}-i g_{j} \pi_{j} B_{\mu, j}+\mathcal{O}\left(\frac{\pi_{j}^{2}}{v_{j}^{2}}\right) \\
& \sim \partial_{\mu} H^{\alpha} T^{\alpha}+i g_{e f f}^{\prime}\left[B_{\mu}^{(0)}, H\right]=T^{\alpha}\left(\partial_{\mu} H^{\alpha}+i g_{\text {eff }}^{\prime} i f_{\alpha 8 \gamma} B_{\mu}^{(0), 8} H^{\gamma}\right),
\end{aligned}
$$

onde denotamos o bóson de gauge correspondente ao gerador $T^{8}$ por $B_{\mu, j}$ e seu modo zero por $B_{\mu}^{(0)}$. O símbolo " " na segunda linha indica que tomamos apenas a parte que contém os modos zero da expressão anterior. Das constantes de estrutura, (4.3) vemos que o acoplamento será proporcional a $f_{678}=\sqrt{3} / 2$, assim, devemos escolher os acoplamentos de gauge de maneira que $g_{\text {eff }}^{\prime}=g^{\prime} / \sqrt{3}$, onde $g^{\prime}$ é o acoplamento de $U(1)_{Y}$ do MP para que a hipercarga do bóson de Higgs seja $Y_{h}=1 / 2$. Como temos cinco acoplamentos de gauge, $g_{0}, g_{0}^{\prime}, g_{N}, g_{N}^{\prime}$ e $\tilde{g}$, sempre temos liberdade suficiente para obter $g$ e $g^{\prime}$ do MP.

Sem perda de generalidade, podemos usar a invariância sob a simetria $S U(2) \times$ $U(1)$ não quebrada para escolher uma das componentes de $H^{\alpha}$ como o bóson de Higgs que adquirirá um vev, que denotaremos simplesmente por $h$ e as outras como os modos de NG que darão as componentes longitudinais dos bósons $W$ e $Z$. Escolhendo por exemplo $h \equiv H^{6}$, podemos checar que a EWSB se dará 
corretamente expandindo o termo quadrático nos modos zero de $S U(2) \times U(1)$ :

$$
\begin{aligned}
& \sum_{j=1}^{N} \frac{1}{2}\left(g_{j-1} \pi_{j} A_{\mu, j-1}^{a}-g_{j} A_{\mu, j} \pi_{j}\right)^{2} \\
& \sim \sum_{A=1}^{8} \frac{1}{2}\left(g_{\text {eff }}^{a} f_{A a 6} A_{\mu}^{(0), a} h g_{\text {eff }}^{b} f_{A b 6} A_{\mu}^{(0), b} h\right) \\
& =\frac{1}{8} h^{2}\left[g^{2}\left(W_{1}^{(0)}\right)^{2}+g^{2}\left(W_{2}^{(0)}\right)^{2}+\left(g W_{3}^{(0)}-g^{\prime} B^{(0)}\right)^{2}\right] .
\end{aligned}
$$

A expressão acima representa a contribuição de ordem mais baixa para as massas dos modos zero, e é idêntica a Lagrangiana de massas dos bósons de gauge do MP. Portanto, a menos de pequenas correções devidas a mistura com os modos massivos (que discutiremos mais adiante), obteremos as massas dos bósons $W$ e $Z$ corretas se $h$ adquirir um vev: $\langle h\rangle \simeq 246 \mathrm{GeV}$.

A nível árvore, o estado $H^{\alpha}$ não possui massa e de fato todas as suas interações involvem derivadas, já que é um bóson de NG. Para que esse estado seja efetivamente o dubleto de Higgs será necessário gerar radiativamente um potencial com os acoplamentos corretos para que o Higgs tenha um vev não trivial, capaz de efetuar a EWSB. Trataremos desse tópico na seção seguinte.

\subsection{Potencial Efetivo}

Uma vez que os acoplamentos de gauge de $S U(2) \times U(1)$ nas extremidades da cadeia quebram explicitamente a simetria global $S U(3)$ diagonal, o estado $H$ não é um bóson de NG verdadeiro, e pode adquirir um potencial não trivial por correções radiativas. Lembramos que a correção para a massa do escalar devida 
a bósons de gauge é positiva, enquanto que a que vem de férmions é negativa, devido a estatística de Fermi-Dirac [1]. Assim, se quisermos obter uma massa taquiônica para o Higgs, para que ele adquira um vev e realize a quebra da simetria eletro-fraca, as correções radiativas para a sua massa que vêm dos loops de férmions (essencialmente o quark top, devido a seu acoplamento de ordem um com o Higgs) devem ser maiores do que as geradas pelos bósons de gauge. Como este trabalho ainda está em produção [32], ainda não temos um modelo completo, que requer escolher corretamente as representações fermiônicas no quiver para obter uma teoria com os acoplamentos corretos e livre de anomalias, como em [58]. Ainda assim, achamos relevante expor aqui os nossos resultados preliminares, que dizem respeito às correções radiativas geradas pelos bósons de gauge.

Uma maneira conveniente de estudar o efeito dessas correções radiativas é o formalismo do potencial efetivo de Coleman-Weinberg [88]. Vamos revisar brevemente como calcular a ação efetiva a 1-loop e depois aplicar a técnica ao nosso modelo para obter o efeito dos loops de bósons de gauge sobre o potencial do Higgs.

Dada a função de partição do campo $\phi(x)$ em presença da fonte externa $J(x)$ :

$$
Z=\int \mathcal{D} \phi e^{i \int d^{4} x(\mathcal{L}[\phi]+J \phi)} .
$$

Podemos definir $W[J] \equiv-i \log Z$. Esse funcional tem a interpretação de (menos) a energia do vácuo em presença da fonte externa $J, e^{i W[J]}=\left\langle 0\left|e^{-i H T}\right| 0\right\rangle_{J}$. Diferenciando com respeito à fonte externa, obtemos o valor esperado de vácuo de 
$\phi(x)$ em presença da fonte, que chamamos de "campo clássico" $\phi_{c}(x)$ :

$$
\frac{\delta W[J]}{\delta J(x)}=-i \frac{\delta}{\delta J(x)} \log Z=\langle 0|\phi(x)| 0\rangle_{J} \equiv \phi_{c}(x)
$$

De fato, pode-se mostrar que sucessivas derivadas funcionais de $W[J]$ geram as funções conexas de n-pontos [1]. Efetuando a transformada de Legendre:

$$
\Gamma\left[\phi_{c}\right] \equiv W[J]-\int d^{4} x J(x) \phi_{c}(x)
$$

Definimos a ação efetiva, que é o funcional gerador de diagramas 1PI [1]. A ação efetiva pode ser interpretada fazendo uma analogia com a termodinâmica: para sistemas a temperatura nula, a configuração mais estável é aquela que minimiza a energia interna, $U$. Já, em presença de flutuações térmicas, o estado de equilibrio termodinâmico é aquele que minimiza a energia livre $F=U-T S$. Analogamente, a nível clássico a configuração de campo mais estável é a que minimiza a energia (o potencial clássico, se consideramos campos constantes), enquanto que em presença de flutuações quânticas devemos minimizar a ação efetiva $\Gamma\left[\phi_{c}\right]$. De fato:

$$
\frac{\delta \Gamma\left[\phi_{c}\right]}{\delta \phi_{c}(x)}=-J(x)
$$

Assim, fazendo $J=0$, vemos que o valor esperado de vácuo de $\phi$ é obtido minimizando a ação efetiva.

Para calcular a ação efetiva, seguimos o método da integral funcional, devido a Jackiw [91]. Fazemos a mudança de variável $\phi(x)=\phi_{c}(x)+\eta(x)$, onde $\eta(x)$ 
representa as flutuações quânticas em torno do vev $\phi_{c}$ então expandimos:

$$
\begin{aligned}
\mathcal{L}[\phi]+J \phi= & \mathcal{L}\left[\phi_{c}\right]+J \phi_{c}+\int d^{4} x \eta(x)\left(\left.\frac{\delta \mathcal{L}}{\delta \phi(x)}\right|_{\phi=\phi_{c}}+J(x)\right)+ \\
& +\left.\frac{1}{2 !} \int d^{4} x d^{4} y \eta(x) \eta(y) \frac{\delta^{2} \mathcal{L}}{\delta \phi(x) \phi(y)}\right|_{\phi=\phi_{c}}+\mathcal{O}\left(\eta^{3}\right) .
\end{aligned}
$$

Podemos eliminar o termo linear em $\eta(x)$ se escrevermos $J(x)=J_{1}(x)+\delta J(x)$, onde $J_{1}(x)$ é tal que $\phi_{c}(x)$ satisfaz a equação de movimento:

$$
\left.\frac{\delta \mathcal{L}}{\delta \phi(x)}\right|_{\phi=\phi_{c}}+J_{1}(x)=0 .
$$

Fazendo isso, $\delta J(x)$ é interpretado como um contratermo que sempre pode ser escolhido para que o vev de $\phi$ não seja deslocado por flutuações quânticas devido a funções de um ponto (tadpoles) [1]. Truncando a série no termo quadrático em $\eta(x)$, obtemos uma integral funcional quadrática, que podemos avaliar formalmente:

$$
\begin{aligned}
e^{i W[J]} & \simeq \int \mathcal{D} \eta \exp \left\{i \int d^{4} x\left(\mathcal{L}\left[\phi_{c}\right]+J \phi_{c}+\left.\frac{1}{2} \int d^{4} y \eta(x) \eta(y) \frac{\delta^{2} \mathcal{L}}{\delta \phi(x) \phi(y)}\right|_{\phi_{c}}\right)\right\} \\
& =\exp \left\{i \int d^{4} x\left(\mathcal{L}\left[\phi_{c}\right]+J \phi_{c}\right)-\frac{1}{2} \ln \operatorname{Det}\left[\frac{\delta^{2} \mathcal{L}}{\delta \phi \delta \phi}\right]_{\phi_{c}}\right\}
\end{aligned}
$$

onde escrevemos $\operatorname{Det}\left[\delta^{2} \mathcal{L}\right]^{-1 / 2}=\exp \left\{-1 / 2 \ln \operatorname{Det}\left[\delta^{2} \mathcal{L}\right]\right\}$. Essa aproximação é equivalente a avaliar a ação efetiva a 1-loop [91]. Podemos então efetuar a transformada de Legendre, obtendo:

$$
\Gamma\left[\phi_{c}\right]=\int d^{4} x\left(\mathcal{L}\left[\phi_{c}\right]\right)+\frac{i}{2} \ln \operatorname{Det}\left[\frac{\delta^{2} \mathcal{L}}{\delta \phi \delta \phi}\right]_{\phi_{c}} .
$$


Antes de prosseguir, notamos que, para férmions, a integral de trajetória grassmaniana (ou equivalentemente, o sinal negativo do loop fermiônico) forneceria $\exp \left\{-i \ln \operatorname{Det}\left[\delta^{2} \mathcal{L}\right]\right\}$ e portanto férmions contribuem com o sinal oposto para a ação efetiva, o que é crucial para a quebra espontânea de simetria. Se expandirmos a ação efetiva em potências do momento:

$$
\Gamma\left[\phi_{c}\right]=\int d^{4} x\left[-V_{e f}\left[\phi_{c}\right]+Z\left[\phi_{c}\right]\left(\partial_{\mu} \phi_{c}\right)^{2}+\cdots\right]
$$

Vemos que, para configurações de campo constantes, correspondentes a valores esperados de vácuo invariantes sob o grupo de Poincaré, o vev de $\phi$ é obtido minimizando a função:

$$
\frac{\partial V_{e f}\left(\phi_{c}\right)}{\partial \phi_{c}}=0
$$

Por essa razão, a função $V_{e f}$ é chamada de potencial efetivo e representa a densidade de energia para campos constantes, levando em conta as correções quânticas. A questão da quebra espontânea de simetria na presença de flutuações quânticas se reduz então ao cálculo de $V_{e f}$. Para sermos mais concretos, para uma teoria escalar $\mathcal{L}=\frac{1}{2}\left(\partial_{\mu} \phi\right)^{2}-V[\phi]$, obtemos, para campos constantes:

$$
\Gamma\left[\phi_{c}\right]=-\int d^{4} x V\left(\phi_{c}\right)+\frac{i}{2} \ln \operatorname{Det}\left[\partial^{2}+V^{\prime \prime}\left(\phi_{c}\right)\right]
$$


Como $\phi_{c}$ não depende de $x$, podemos avaliar o segundo termo no espaço de momentos:

$$
\frac{i}{2} \ln \operatorname{Det}\left[\partial^{2}+V^{\prime \prime}\left(\phi_{c}\right)\right]=\frac{i}{2} \operatorname{Tr} \ln \left[\partial^{2}+V^{\prime \prime}\left(\phi_{c}\right)\right]=\frac{i}{2} \int \frac{d^{4} k}{2 \pi^{4}} \ln \left[-k^{2}+V^{\prime \prime}\left(\phi_{c}\right)\right],
$$

obtendo o potencial efetivo:

$$
V_{e f}\left(\phi_{c}\right)=V\left(\phi_{c}\right)-\frac{i}{2} \int \frac{d^{4} k}{2 \pi^{4}} \ln \left[-k^{2}+V^{\prime \prime}\left(\phi_{c}\right)\right]
$$

O segundo termo tem a interpretação da energia de vácuo em presença do campo de fundo $\phi_{c}$, e em particular, $V^{\prime \prime}\left(\phi_{c}\right)$ é a massa efetiva nesse fundo. No caso de muitos campos, naturalmente generalizamos para a matriz de massas $V^{\prime \prime}\left(\phi_{c}\right) \rightarrow$ $\left.\partial_{i} \partial_{j} V\left(\phi^{k}\right)\right|_{\phi^{k}=\phi_{c}^{k}}$, cujos autovalores são as massas dos campos que são adquiridas devido ao vev de $\phi$. Devemos então somar as contribuições de todos os graus de liberdade ao potencial.

Podemos agora aplicar esse formalismo para obter o efeito das correções radiativas dos bósons de gauge para o potencial do Higgs em nosso modelo. Trabalharemos no gauge $\partial_{\mu} A^{\mu}=0$, por razões que ficarão claras em breve. A Lagrangiana do modelo é dada por:

$$
\begin{aligned}
\mathcal{L} & =\sum_{j=0}^{N}\left\{-\frac{1}{2} \operatorname{Tr}\left[F_{\mu \nu, j} F_{j}^{\mu \nu}\right]-\frac{1}{\xi} \operatorname{Tr}\left[\left(\partial_{\mu} A_{j}^{\mu}\right)^{2}\right]-\bar{\eta}_{j}^{A} \partial_{\mu} D_{A B}^{\mu} \eta_{j}^{B}\right\}+ \\
& +\sum_{j=1}^{N}\left\{\operatorname{Tr}\left[\left(\partial_{\mu} \Phi_{j}\right)^{\dagger} \partial^{\mu} \Phi_{j}\right]+\operatorname{Tr}\left[i\left(\partial^{\mu} \Phi_{j}\right)^{\dagger}\left(g_{j-1} A_{\mu, j-1} \Phi_{j}-g_{j} \Phi_{j} A_{\mu, j}\right)+\text { h.c. }\right]\right. \\
& \left.+\frac{v_{j}^{2}}{2}\left(g_{j}^{2} A_{\mu, j}^{A 2}+g_{j-1}^{2} A_{\mu, j-1}^{A 2}\right)-2 g_{j} g_{j-1} \operatorname{Tr}\left[\Phi_{j}^{\dagger} A_{\mu, j-1} \Phi_{j} A_{j}^{\mu}\right]\right\}
\end{aligned}
$$


onde $\mathcal{L}_{\eta}=-\bar{\eta}_{j}^{A} \partial_{\mu} D_{A B}^{\mu} \eta_{j}^{B}$ é a Lagrangiana de Fadeev-Popov nesse gauge [1]. O termo cinético dos $\Phi_{j}$ pode ser reescrito convenientemente como:

$$
\operatorname{Tr}\left[\left(\partial_{\mu} \Phi_{j}\right)^{\dagger} \partial^{\mu} \Phi_{j}\right]=\frac{1}{v_{j}^{2}} \operatorname{Tr}\left[\left(\Phi_{j}^{\dagger} \partial_{\mu} \Phi_{j}\right)^{\dagger} \Phi_{j}^{\dagger} \partial^{\mu} \Phi_{j}\right]
$$

que é uma forma equivalente, devido à ciclicidade do traço e à identidade $\Phi_{j}^{\dagger} \Phi_{j}=$ $v_{j}^{2}$. Expandindo os $\Phi_{j}=v_{j}\left(\mathbf{1}+i \frac{\pi_{j}}{v_{j}}-\frac{1}{2 !}\left(\frac{\pi_{j}}{v_{j}}\right)^{2}+\cdots\right)$, após algum trabalho algébrico, obtemos:

$$
\begin{aligned}
\Phi_{j}^{\dagger} \partial^{\mu} \Phi_{j} & =i v_{j} \partial_{\mu} \pi_{j}+\frac{1}{2 !}\left[\pi_{j}, \partial_{\mu} \pi_{j}\right]-\frac{i}{3 !} \frac{1}{v_{j}}\left[\pi_{j},\left[\pi_{j}, \partial_{\mu} \pi_{j}\right]\right]+\frac{i}{4 !} \frac{1}{v_{j}^{2}}\left[\pi_{j}\left[\pi_{j},\left[\pi_{j}, \partial_{\mu} \pi_{j}\right]\right]\right]+\cdots \\
& =i v_{j} T^{A}\left(\delta_{A B}+\frac{1}{2 !} \Pi_{A B, j}+\frac{1}{3 !} \Pi_{A B, j}^{2}+\frac{1}{4 !} \Pi_{A B, j}^{3}+\cdots\right) \partial_{\mu} \pi_{j}^{B} \\
& =i v_{j} T^{A} \frac{\left(e^{\Pi_{j}}-1\right)}{\Pi_{j}}{ }_{A B} \partial_{\mu} \pi_{j}^{B} \equiv i v_{j} T^{A} E_{B}^{A}{ }_{B}\left(\pi_{j}\right) \partial_{\mu} \pi_{j}^{B}
\end{aligned}
$$

onde definimos as matrizes $\frac{f_{A C B} \pi_{j}^{C}}{v_{j}} \equiv \Pi_{A B, j}$, e $E_{B}^{A}{ }_{B}\left(\pi_{j}\right) \equiv \frac{\left(e^{\Pi_{j}}-1\right)}{\Pi_{j}}$. Similarmente:

$$
\begin{aligned}
\operatorname{Tr}\left[\Phi_{j}^{\dagger} A_{\mu, j-1} \Phi_{j} A_{j}^{\mu}\right] & =v_{j}^{2} \operatorname{Tr}\left[A_{\mu, j-1} A_{j}^{\mu}\right]+i v_{j} \operatorname{Tr}\left[\left[A_{\mu, j-1}, \pi_{j}\right] A_{j}^{\mu}\right]+ \\
& +\frac{1}{2} \operatorname{Tr}\left[\left[A_{\mu, j-1}, \pi_{j}\right]\left[A_{j}^{\mu}, \pi_{j}\right]\right]+\frac{i}{3 !} \frac{1}{v_{j}} \operatorname{Tr}\left[\pi_{j}\left[\left[A_{\mu, j-1}, \pi_{j}\right],\left[A_{j}^{\mu}, \pi_{j}\right]\right]\right]+\cdots \\
& =\frac{v_{j}^{2}}{2} A_{\mu, j-1}^{A}\left(\delta_{A B}-\Pi_{A B, j}+\frac{1}{2 !} \Pi_{A B, j}^{2}-\frac{1}{3 !} \Pi_{A B, j}^{3}+\cdots\right) A_{j}^{\mu, B} \\
& =\frac{v_{j}^{2}}{2} A_{\mu, j-1}^{A} e_{A B}^{-\Pi_{j}} A_{j}^{\mu, B} .
\end{aligned}
$$


Substituindo essas expansões em (4.33), e expandindo o termo cinético dos $A_{\mu, j}$, obtemos:

$$
\begin{aligned}
\mathcal{L} & =\frac{1}{2} \sum_{j=0}^{N}\left\{A_{\nu, j}^{A}\left(g^{\mu \nu} \partial^{2}-\left(1-\frac{1}{\xi}\right) \partial^{\mu} \partial^{\nu}\right) A_{\mu, j}^{A}+\text { int. }+\mathcal{L}_{\eta}\right\}+ \\
& +\sum_{j=1}^{N}\left\{\frac{1}{2} E_{C}^{A}\left(\pi_{j}\right) E^{A}{ }_{D}\left(\pi_{j}\right) \partial_{\mu} \pi_{j}^{C} \partial^{\mu} \pi_{j}^{D}+v_{j} \partial^{\mu} \pi_{j}^{A} E_{B}^{A}\left(\pi_{j}\right)\left(g_{j-1} A_{\mu, j-1}^{B}-g_{j} A_{\mu, j}^{B}\right)\right. \\
& \left.+\frac{v_{j}^{2}}{2}\left(g_{j}^{2} A_{\mu, j}^{A 2}+g_{j-1}^{2} A_{\mu, j-1}^{A 2}\right)-v_{j}^{2} g_{j} g_{j-1} A_{\mu, j-1}^{A} e_{A B}^{-\Pi_{j}} A_{j}^{\mu, B}\right\}
\end{aligned}
$$

onde o termo int. se refere as interações entre bósons de gauge. A matriz $E^{A}{ }_{B}$ é efetivamente um vielbein na variedade $G / H$, de maneira que podemos definir uma métrica (em geral curva) nesse espaço por $g_{A B} \equiv E^{C}{ }_{A} E^{D}{ }_{B} \delta_{C D}[92,93,94]$. De fato, a Lagrangiana quiral que descreve os bósons de NG deve ser invariante por transformações gerais das "coordenadas" $\pi_{j}^{A}$.

Podemos agora aplicar o formalismo expandindo a Lagrangiana em torno dos valores esperados de vácuo dos campos e mantendo os termos até segunda ordem. Vamos assumir que apenas a combinação linear $H^{\alpha}$ adquire um vev, já que outras combinações lineares são bósons de NG exatos e não podem adquirir um potencial. Podemos usar a unitariedade da expansão em autoestados de (4.15) para escrever:

$$
\pi_{j}^{\alpha}=\frac{q^{N-j}}{\sqrt{\sum_{j=1}^{N} q^{2(j-1)}}} H^{\alpha}+\text { outros estados. }
$$


Escrevemos então $H^{\alpha}=\tilde{H}^{\alpha}+\left\langle H^{\alpha}\right\rangle$, com o vev independente de $x$, e calculamos a derivada funcional:

$$
\begin{aligned}
\frac{1}{2 !} \int d^{4} x d^{4} y & \left(\tilde{A}_{\mu, j}^{A}(x) \tilde{A}_{\nu, k}^{B}(y) \frac{\delta^{2} \mathcal{L}}{\delta A_{\mu, j}^{A}(x) \delta A_{\nu, k}^{B}(y)}+\right. \\
& \left.+\tilde{A}_{\mu, j}^{A}(x) \tilde{\pi}_{k}^{B}(y) \frac{\delta^{2} \mathcal{L}}{\delta A_{\mu, j}^{A}(x) \delta \pi_{k}^{B}(y)}+\tilde{\pi}_{j}^{A}(x) \tilde{\pi}_{k}^{B}(y) \frac{\delta^{2} \mathcal{L}}{\delta \pi_{j}^{A}(x) \delta \pi_{k}^{B}(y)}\right)\left.\right|_{H^{\alpha}=\left\langle H^{\alpha}\right\rangle},
\end{aligned}
$$

onde está implicita a soma em índices de gauge e do quiver. Notamos que $\mathcal{L}_{\eta}$ não contribui no gauge $\partial_{\mu} A^{\mu}=0$. Por simplicidade de notação, omitiremos as tildes nos campos daqui adiante.

Vamos considerar primeiramente a contribuição do termo cinético dos $\pi_{j}^{A}$. Para que a integral funcional sobre os $\pi_{j}^{A}$ seja invariante por reparametrizações dos bósons NG, devemos incluir o determinante da métrica $g \equiv\left|\operatorname{Det}\left[g_{A B}\right]\right|$ na medida de integração $[93,94]$. Assim, a contribuição do termo cinético a ordem mais baixa (1-loop) será:

$$
\left.\int \mathcal{D} \pi_{j}^{A} \sqrt{g}\right|_{H^{\alpha}=\left\langle H^{\alpha}\right\rangle} e^{i \int d^{4} x \sum_{j=1}^{N} \frac{1}{2} g_{A B}\left(\left\langle H^{\alpha}\right\rangle\right) \partial_{\mu} \pi_{j}^{A} \partial^{\mu} \pi_{j}^{B}}
$$

onde indicamos que a ordem mais baixa, a contribuição para o potencial efetivo que vem do determinante da métrica é obtido avaliando a métrica no vev dos campos. De fato, a essa ordem, o determinante contribui a 1-loop. Isso pode ser visto mais facilmente se lembrarmos que a expansão em potências de $\hbar$ corresponde a expansão em loops [95], assim, se restaurarmos as dimensões de $\hbar$ para a ação e expandirmos $S=S_{0}+\hbar S_{1}+\ldots$, onde $S_{0}$ é a contribuição a nível árvore, 
$\hbar S_{1}$ a correção a 1-loop, etc, obtemos:

$$
\sqrt{g} e^{i\left(\frac{S_{0}}{\hbar}+S_{1}+\ldots\right)}=e^{\frac{1}{2} \ln \operatorname{Det}\left[g_{A B}\right]} e^{i\left(\frac{S_{0}}{\hbar}+S_{1}+\ldots\right)} .
$$

Vemos que o determinante da métrica avaliado no vev contribui uma correção para a ação efetiva a 1-loop dado por $\Delta S_{1} \equiv \frac{1}{2} \ln \operatorname{Det}\left[g_{A B}\left(\left\langle H^{\alpha}\right\rangle\right)\right]$, o que é consistente com a equação (4.27). Efetuamos a integral funcional gaussiana sobre os $\pi_{j}^{A}:$

$$
\begin{aligned}
& \int \mathcal{D} \pi_{j}^{A} \sqrt{g\left(\left\langle H^{\alpha}\right\rangle\right)} e^{i \int d^{4} x \sum_{j=1}^{N} \frac{1}{2} g_{A B}\left(\left\langle H^{\alpha}\right\rangle\right) \partial_{\mu} \pi_{j}^{A} \partial^{\mu} \pi_{j}^{B}} \\
& \sim \frac{\sqrt{g\left(\left\langle H^{\alpha}\right\rangle\right)}}{\sqrt{g\left(\left\langle H^{\alpha}\right\rangle\right) \operatorname{Det}\left[-k^{2}\right]}}=\frac{1}{\sqrt{\operatorname{Det}\left[-k^{2}\right]}} .
\end{aligned}
$$

Observamos, portanto, que o termo cinético não irá contribuir a 1-loop, pois a contribuição do determinante cancela exatamente a dependência em $\left\langle H^{\alpha}\right\rangle$ a essa ordem $[93,94]$. A contribuição dos bósons de gauge calculada a partir de (4.39) será dada por:

$$
\begin{aligned}
\mathcal{L}_{A} & =\frac{1}{2} \sum_{j=0}^{N} A_{\nu, j}^{A}\left(g^{\mu \nu} \partial^{2}-\left(1-\frac{1}{\xi}\right) \partial^{\mu} \partial^{\nu}\right) A_{\mu, j}^{A}+ \\
& +\sum_{j=1}^{N}\left\{v_{j} \partial^{\mu} \pi_{j}^{A} E_{B}^{A}\left(\left\langle H^{\alpha}\right\rangle\right)\left(g_{j-1} A_{\mu, j-1}^{B}-g_{j} A_{\mu, j}^{B}\right)\right. \\
& \left.+\frac{v_{j}^{2}}{2}\left(g_{j}^{2} A_{\mu, j}^{A 2}+g_{j-1}^{2} A_{\mu, j-1}^{A 2}\right)-v_{j}^{2} g_{j} g_{j-1} A_{\mu, j-1}^{A} \exp \left(-\frac{q^{N-j} f_{A \beta B}}{v_{j} \sqrt{\sum_{j=1}^{N} q^{2(j-1)}}}\left\langle H^{\beta}\right\rangle\right) A_{j}^{\mu, B}\right\} .
\end{aligned}
$$


Como trabalhamos no gauge $\partial_{\mu} A^{\mu}=0$, o termo da segunda linha é nulo se integrarmos por partes, já que $E_{B}^{A}\left(\left\langle H^{\alpha}\right\rangle\right)$ é uma matriz constante. ${ }^{3}$ A terceira linha é então a matriz de massas dos bósons de gauge em presença do campo de fundo $\left\langle H^{\alpha}\right\rangle$. Como na seção anterior, vamos escolher a orientação do vev de $H$ na direção de $T^{6},\left\langle H^{6}\right\rangle \equiv\langle h\rangle$. Para simplificar a notação, denotaremos:

$$
\begin{aligned}
& \exp \left(-\frac{q^{N-j} f_{A 6 B}}{v_{j} \sqrt{\sum_{j=1}^{N} q^{2(j-1)}}}\langle h\rangle\right) \equiv e_{A B}^{-\frac{H}{v_{j}^{h}}}, \\
& v_{j}^{h} \equiv v_{j} \frac{\sqrt{\sum_{j=1}^{N} q^{2(j-1)}}}{q^{N-j}} .
\end{aligned}
$$

Para obter a contribuição dos bósons de gauge para o potencial efetivo, escolhemos $\xi=1$ (gauge de Feynman) e efetuamos a integral funcional quadrática sobre os bósons de gauge:

$$
\int \mathcal{D} A_{j}^{\mu} \exp \left[i \int d^{4} x\left(\frac{1}{2} \sum_{j, k=0}^{N} A_{\nu, j}^{A}\left(g^{\mu \nu} \delta_{A B} \delta_{j, k} \partial^{2}+M_{A}^{2}(h)\right) A_{\mu, k}^{B}\right)\right]
$$

Em analogia ao caso escalar, obtemos a expressão para $V_{e f}(h)$ (lembrando que o potencial é nulo a nível árvore):

$$
V_{e f}(h)=-\frac{i 3}{2} \int \frac{d^{4} k}{2 \pi^{4}} \operatorname{Tr}\left[\ln \left(-k^{2}+M_{A}^{2}(h)\right)\right],
$$

\footnotetext{
${ }^{3}$ Mais precisamente, nesse gauge o propagador dos bósons é transverso, ou seja, satisfaz $k_{\mu} \Delta^{\mu \nu}\left(k^{2}\right)=0$, eliminando a contribuição dos diagramas com os vértices da segunda linha de (4.43) para $V_{e f}[88,95]$.
} 
onde o fator de 3 corresponde às três polarizações dos bósons de gauge massivos. Para avaliar a integral, efetuamos a rotação de Wick $k_{0} \rightarrow i k_{4}, k^{2} \rightarrow-k_{E}^{2}$. A integral é formalmente divergente, assim, devemos adotar um procedimento de regularização. Mostraremos adiante que para $N \geq 2$, o potencial é finito e portanto o resultado independe da regularização, assim, para mostrar o cancelamento da divergência quadrática de maneira intuitiva, regularizamos integrando o momento euclidiano até um corte $\Lambda$. Obtemos assim o resultado clássico de Coleman-Weinberg [88]:

$$
V_{e f}(h)=\frac{3 \Lambda^{2}}{32 \pi^{2}} \operatorname{Tr}\left[M_{A}^{2}(h)\right]+\frac{3}{64 \pi^{2}} \operatorname{Tr}\left[\left(M_{A}^{2}(h)\right)^{2}\left(\ln \frac{M_{A}^{2}(h)}{\Lambda^{2}}-\frac{1}{2}\right)\right],
$$

onde omitimos termos independentes de $h$, que apenas definem o zero da energia (constante cosmológica) assim como termos que vão a zero no limite $\Lambda \rightarrow \infty$. Podemos absorver o fator de $1 / 2$ redefinindo o corte $\Lambda^{2} \rightarrow \Lambda^{2} e^{1 / 2}$.

Explicitamente, vemos de (4.43) que a matriz de massas tem a forma:

$$
\begin{aligned}
& M_{A}^{2}(h) / v^{2}= \\
& \left(\begin{array}{cccccc}
g_{0}^{2} q^{2} \delta_{A B} & -g_{0} \tilde{g} q^{2} e_{A B}^{-\frac{H}{v_{1}^{h}}} & 0 & \cdots & 0 & 0 \\
-g_{0} \tilde{g} q^{2} e_{A B}^{-\frac{H^{t}}{v_{1}^{h}}} & \tilde{g}^{2}\left(q^{2}+q^{4}\right) \delta_{A B} & -\tilde{g}^{2} q^{4} e_{A B}^{-\frac{H}{v_{2}^{h}}} & \cdots & 0 & 0 \\
0 & -\tilde{g}^{2} q^{4} e_{A B}^{-\frac{H^{t}}{v_{2}^{h}}} & \tilde{g}^{2}\left(q^{4}+q^{6}\right) \delta_{A B} & \cdots & 0 & 0 \\
\vdots & \vdots & \vdots & \ddots & \vdots & \vdots \\
0 & 0 & 0 & \cdots & \tilde{g}^{2}\left(q^{2(N-1)}+q^{2 N}\right) \delta_{A B} & -g_{N} \tilde{g} q^{2 N} e_{A B}^{-\frac{H}{v_{N}^{h}}} \\
0 & 0 & 0 & \cdots & -g_{N} \tilde{g} q^{2 N} e_{A B}^{-\frac{H^{t}}{v_{N}^{h}}} & g_{N}^{2} q^{2 N} \delta_{A B}
\end{array}\right)
\end{aligned}
$$


Quando o índice de gauge for $A=8$, devemos substituir $g_{0, N} \rightarrow g_{0, N}^{\prime}$ nas expressões. É fácil ver de (4.48) que $\operatorname{Tr}\left[M_{A}^{2}(h)\right]$ não depende de $h$. Essa propriedade implica na ausência de divergências quadráticas para o potencial, resolvendo o problema da hierarquia. Para $N \geq 2$, pode-se mostrar que $\operatorname{Tr}\left[\left(M_{A}^{2}(h)\right)^{2}\right]$ também independe do vev do Higgs [87], o que elimina a divergência logaritmica e torna o potencial finito a 1-loop, como em modelos em $A d S_{5}{ }^{4}$

Obter um valor explícito para os termos de massa e quárticos requer diagonalizar a matriz de massas $M_{A}^{2}(h)$. Para modelos em $A d S_{5}$ é possível re-escrever o potencial efetivo em termos de um fator de forma que depende apenas de propriedades analíticas das autofunções (2.27) e pode ser calculado explicitamente [86]. No nosso caso, é provável que seja possível utilizar propriedades análogas das funções de q-Bessel para obter uma expressão analítica, mas até o momento, só pudemos avaliar o espectro numericamente.

Vamos analizar em mais detalhe as características qualitativas do espectro de (4.48). Para uma teoria com $N+1$ sítios, essa matriz possui $8 N$ autovalores, que se dividem em torres de modos massivos após a EWSB. Como vimos na sessão anterior, a escolha $h=\left\langle H^{6}\right\rangle$ define a orientação dos geradores de $S U(2)_{L} \times U(1)_{Y}$ em $S U(3)$. Utilizando a álgebra de comutadores, (4.4), os geradores se dividem em "carregados" $T^{1}, T^{2}, T^{4}, T^{5}$ e "neutros" $T^{3}, T^{6}, T^{7}, T^{8}$, que não se misturam sob a ação das rotações $\exp \left(-\frac{H}{v_{j}^{h}}\right)_{A B}$. Além disso, a matriz $f_{A \beta B} H^{\beta}$ possui dois autovalores nulos, que não são afetados pelo vev de $H$. Um deles é o gerador $T^{6}$, pela nossa escolha da orientação do vev. Mais geralmente, uma combinação linear dos $X^{\alpha}$ não se mistura com outros modos massivos após a EWSB. Como

\footnotetext{
${ }^{4}$ De maneira geral, para $N+1$ sítios, a dependência em $\langle H\rangle$ começa na potência $\operatorname{Tr}\left[\left(M_{A}^{2}(h)\right)^{N+1}\right]$.
} 
os bósons desse setor não tem modo zero, esse gerador corresponde a uma torre de estados pesados. A outra combinação linear que não é afetada pelo vev pertence ao conjunto de geradores $Y^{a}$ e será identificada com o fóton e seus modos massivos, já que a simetria $U(1)_{Q}$ permanece intacta, como vimos no fim da seção anterior.

Obtivemos o espectro e o potencial efetivo numericamente para o caso mais simples que gera um potencial finito, o modelo de três sítios $(N=2)$. Antes de apresentar os resultados numéricos para o espectro e potencial, é interessante calcular a massa dos bósons $W$ e $Z$ utilizando teoria de perturbações. Re-escrevemos a matriz de massas como:

$$
\sum_{j=1}^{N}\left[\frac{v_{j}^{2}}{2}\left(g_{j} A_{\mu, j}^{A}-g_{j-1} A_{\mu, j-1}^{A}\right)^{2}-v_{j}^{2} g_{j} g_{j-1} A_{\mu, j-1}^{A}\left(e_{A B}^{-\frac{H}{v_{j}^{h}}}-\delta_{A B}\right) A_{j}^{\mu, B}\right]
$$

O primeiro termo é a matriz de massas (3.14) não perturbada, que pode ser diagonalizada exatamente, fornecendo o espectro de modos massivos antes da EWSB. O segundo termo é a perturbação, que podemos expandir no parâmetro $\langle h\rangle / v$, que assumimos pequeno. Uma simplificação importante é obtida fazendo a separação em termos pares e ímpares:

$$
\begin{aligned}
& -v_{j}^{2} g_{j} g_{j-1} A_{\mu, j-1}^{A}\left(e_{A B}^{-\frac{H}{v_{j}^{h}}}-\delta_{A B}\right) A_{j}^{\mu, B}= \\
& -v_{j}^{2} g_{j} g_{j-1}\left(A_{\mu, j-1}^{A}\left(\cosh \left(\frac{H}{v_{j}^{h}}\right){ }_{A B}-\delta_{A B}\right) A_{j}^{\mu, B}-A_{\mu, j-1}^{A} \operatorname{senh}\left(\frac{H}{v_{j}^{h}}\right)_{A B} A_{j}^{\mu, B}\right) .
\end{aligned}
$$


Utilizando a álgebra de Lie (4.4), vemos que as potências pares de comutadores (termo $\cosh (H)-1)$ são diagonais nos geradores, ou seja, acoplam os $X^{\alpha}$ com os $X^{\alpha}$ e os $Y^{a}$ com os $Y^{a}$, enquanto que as potências ímpares (termo $\operatorname{senh}(H)$ ) misturam $X^{\alpha}$ e $Y^{a}$. Uma vez que os bósons de gauge associados aos geradores $X^{\alpha}$ não têm modo zero, eles adquirem massas grandes (de ordem $\tilde{g} v \sqrt{q^{2}+q^{4}} \sim$ $10^{7} \mathrm{TeV}$ para $N=2$ ), e podem ser integrados fora com segurança.

Vamos considerar a expressão para a matriz par no setor $\operatorname{dos} Y^{a}$. O termo $\sim\left(A_{j}^{1}\langle h\rangle\right)^{2}$ por exemplo se escreve:

$$
\begin{aligned}
& \cosh \left(\frac{H}{v_{j}^{h}}\right)_{11}-1=\frac{1}{2 !}\left(\frac{\langle h\rangle}{v_{j}^{h}}\right)^{2}\left(f_{165} f_{561}\right)+\frac{1}{4 !}\left(\frac{\langle h\rangle}{v_{j}^{h}}\right)^{4}\left(f_{165} f_{561}\right)^{2}+\cdots \\
& =-\frac{1}{2 !}\left(\frac{\langle h\rangle}{v_{j}^{h}}\right)^{2} f_{165}^{2}+\frac{1}{4 !}\left(\frac{\langle h\rangle}{v_{j}^{h}}\right)^{4} f_{165}^{4}+\cdots \\
& =-\frac{1}{2 !}\left(\frac{\langle h\rangle}{2 v_{j}^{h}}\right)^{2}+\frac{1}{4 !}\left(\frac{\langle h\rangle}{2 v_{j}^{h}}\right)^{4}+\cdots=\cos \left(\frac{\langle h\rangle}{2 v_{j}^{h}}\right)-1,
\end{aligned}
$$

onde usamos $f_{165}=1 / 2$. Notamos que a série passou de $\cosh (x)$ para $\cos (x / 2)$. Prosseguindo dessa maneira, a matriz par será dada por:

$$
\begin{gathered}
\cosh \left(\frac{H}{v_{j}^{h}}\right){ }_{A B}-\delta_{A B}= \\
\left(\begin{array}{cccc}
\cos \left(\frac{\langle h\rangle}{2 v_{j}^{h}}\right)-1 & 0 & 0 & 0 \\
0 & \cos \left(\frac{\langle h\rangle}{2 v_{j}^{h}}\right)-1 & 0 & 0 \\
0 & 0 & \frac{1}{4}\left(\cos \left(\frac{\langle h\rangle}{v_{j}^{h}}\right)+3\right)-1 & -\frac{\sqrt{3}}{4}\left(\cos \left(\frac{\langle h\rangle}{v_{j}^{h}}\right)-1\right) \\
0 & 0 & -\frac{\sqrt{3}}{4}\left(\cos \left(\frac{\langle h\rangle}{v_{j}^{h}}\right)-1\right) & \frac{1}{4}\left(3 \cos \left(\frac{\langle h\rangle}{v_{j}^{h}}\right)+1\right)-1
\end{array}\right) .
\end{gathered}
$$


Nessa matriz vemos dois termos na diagonal correspondentes aos geradores $T^{1} \mathrm{e}$ $T^{2}$ mais um bloco que mistura os geradores $T^{3}$ e $T^{8}$, como esperamos da EWSB. Seus autovalores são $\left\{0, \cos \left(\frac{\langle h\rangle}{v_{j}^{h}}\right)-1, \cos \left(\frac{\langle h\rangle}{2 v_{j}^{h}}\right)-1, \cos \left(\frac{\langle h\rangle}{2 v_{j}^{h}}\right)-1\right\}$. O autovalor nulo corresponde à simetria $U(1)_{Q}$ não quebrada.

Usando essa matriz, podemos escrever a matriz de massas dos bósons carregados $W_{j}^{ \pm}=\left(A_{j}^{1} \mp i A_{j}^{2}\right) / \sqrt{2}$, assim como a matriz dos bósons neutros $A_{j}^{3}, A_{j}^{8}$. Para $N=2$, por exemplo, a matriz dos bósons carregados é escrita:

$$
\begin{aligned}
& M_{c}^{2}= \\
& v^{2}\left(\begin{array}{ccc}
g_{0}^{2} q^{2} & -g_{0} \tilde{g} q^{2} \cos \left(\frac{\langle h\rangle}{2 v \sqrt{q^{2}+1}}\right) & 0 \\
-g_{0} \tilde{g} q^{2} \cos \left(\frac{\langle h\rangle}{2 v \sqrt{q^{2}+1}}\right) & \tilde{g}^{2}\left(q^{2}+q^{4}\right) & -g_{2} \tilde{g} q^{4} \cos \left(\frac{\langle h\rangle}{2 v q^{2} \sqrt{q^{2}+1}}\right) \\
0 & -g_{2} \tilde{g} q^{4} \cos \left(\frac{\langle h\rangle}{2 v q^{2} \sqrt{q^{2}+1}}\right) & g_{2}^{2} q^{4}
\end{array}\right) .
\end{aligned}
$$

Usando a expressão dos modos zero não perturbados (4.8), podemos avaliar a massa dos bósons $W$ e $Z$ físicos perturbativamente, obtendo as expressões:

$$
\begin{aligned}
& M_{W}^{2}=\frac{g^{2}\langle h\rangle^{2}}{4}\left(1-\frac{1}{48} \frac{\langle h\rangle^{2}}{v_{N}^{2}} \frac{\left(q^{2}-1\right)\left(1+q^{2 N}+q^{4 N}\right)}{\left(1+q^{4}+q^{2}\right)\left(q^{2 N}-1\right)}+\mathcal{O}\left(\frac{\langle h\rangle^{4}}{v^{4}}\right)\right), \\
& M_{Z}^{2}=\frac{\left(g^{2}+g^{\prime 2}\right)\langle h\rangle^{2}}{4}\left(1-\frac{1}{12} \frac{\langle h\rangle^{2}}{v_{N}^{2}} \frac{\left(q^{2}-1\right)\left(1+q^{2 N}+q^{4 N}\right)}{\left(1+q^{4}+q^{2}\right)\left(q^{2 N}-1\right)}+\mathcal{O}\left(\frac{\langle h\rangle^{4}}{v^{4}}\right)\right) .
\end{aligned}
$$

Podemos utilizar essas expressões mais (3.112) para calcular a contribuição para o parâmetro T (análoga a obtida na equação (3.126)):

$$
T=\frac{4 \pi}{e^{2}}\left(\frac{1}{16} \frac{\langle h\rangle^{2}}{v_{N}^{2}} \frac{\left(q^{2}-1\right)\left(1+q^{2 N}+q^{4 N}\right)}{\left(1+q^{4}+q^{2}\right)\left(q^{2 N}-1\right)}+\mathcal{O}\left(\frac{\langle h\rangle^{4}}{v^{4}}\right)\right) .
$$


Para $N=2,\langle h\rangle=246 \mathrm{GeV}, v_{N}=v q^{2}=2.3 \mathrm{TeV}$, obtemos $M_{W}=80.37 \mathrm{GeV}$, e o primeiro modo massivo do $W$ tem massa $M_{W}^{(1)} \simeq 3 \mathrm{TeV}$. Para esses valores dos parâmetros, obtemos $T \simeq 0.091\left(\frac{2.3 \mathrm{TeV}}{v_{N}}\right)^{2}$. Notamos que esse resultado não varia significativamente com $N$ para poucos sítios $(q \ll 1)$.

Avaliando numericamente o espectro no modelo de três sítios (incluindo todos os modos massivos), para os parâmetros acima, o espectro de $8 N=16$ bósons de gauge se divide em torres de $N+1=3$ modos massivos para os bósons $W^{ \pm}, Z$ e $\gamma$, totalizando doze modos, sendo as massas do primeiro modo do $W$ e $Z M_{W, Z}^{(1)} \simeq 3.2 \mathrm{TeV}$ e do segundo modo $M_{W, Z}^{(2)} \simeq 2.0 \cdot 10^{11} \mathrm{GeV}$, enquanto que para o fóton temos $M_{\gamma}^{(1)} \simeq 1 \mathrm{TeV}$ e $M_{\gamma}^{(2)} \simeq 9.5 \cdot 10^{10} \mathrm{GeV}$. Os outros quatro estados formam a torre de $N-1=1$ modos para os outros geradores, de massas $M_{X}^{(2)} \simeq 9.1 \cdot 10^{10} \mathrm{GeV}$.

Avaliando numericamente o potencial para $N=2$, obtemos os valores:

$$
V(h)=(256.41 \mathrm{GeV})^{2}\left(\frac{v_{N}}{1 \mathrm{TeV}}\right)^{2} h^{2}+0.030 h^{4}+\mathcal{O}\left(h^{6}\right)
$$

onde indicamos que o termo quadrático escala com $v^{2}$, como esperamos por análise dimensional. Embora ainda não tenhamos calculado as contribuições fermiônicas para o potencial para garantir a $E W S B$, podemos ver se o potencial gerado pelos bósons de gauge está dentro do que esperávamos. Vemos que foi possível gerar radiativamente um potencial com um termo quadrático que é naturalmente menor do que a escala de modos massivos, $v_{N} \sim 1 \mathrm{TeV}$. No entanto, obter um Higgs com massa $m_{h}=125 \mathrm{GeV}$ requer um acoplamento quártico $\lambda \simeq 0.25 \mathrm{em}$ nossa normalização, assim vemos que o termo quártico gerado pelos bósons de gauge é pequeno, o que é um problema genérico de teorias que geram o potencial 
radiativamente.

Outra questão interessante é estudar como o potencial gerado muda com o número de sítios. Ainda não desenvolvemos ferramentas para efetuar esse estudo de maneira sistemática com os métodos numéricos que temos disponíveis, mas estamos trabalhando em desenvolver uma expansão perturbativa tanto em $q$ quanto em $\langle h\rangle / v$ para as massas e autofunções em presença do vev do Higgs (ver [96]). Passamos a apresentar nossas conclusões e considerações para trabalhos futuros. 


\section{Capítulo 5}

\section{Conclusões e Perspectivas}

Apesar do sucesso indiscutível do MP, vimos na introdução que ele ainda apresenta vários problemas em aberto. Em particular, o setor responsável pela quebra espontânea da simetria eletrofraca não é bem compreendido, e o problema da hierarquia de escalas que surge nesse setor sugere que deve existir nova física na escala $\mathrm{TeV}$. Outro problema é a origem da grande hierarquia de massas dos férmions. Nossos estudos se concentraram em buscar soluções para esses problemas sem violar vínculos experimentais importantes na física de sabor e de precisão eletrofraca.

No segundo capítulo, fizemos uma revisão do modelo de dimensões extras curvas de Randall-Sundrum [28], que é capaz de resolver os problemas de hierarquia de massas e de gauge desde que o Higgs esteja localizado próximo da chamada brana infravermelha e os férmions separados no bulk. No entanto, vimos que essa solução sofre de problemas com vínculos da física de sabor, o que nos motiva a estudar uma nova abordagem, chamada desconstrução dimensional $[29,30]$. No capítulo três estudamos o modelo de descontrução dimensional 
com poucos sítios e vimos que ele é capaz de resolver os problemas de hierarquia de maneira similar ao modelo de RS sem, no entanto, violar os vínculos da física de sabor e dos parâmetros de precisão eletrofraca. No quarto capítulo apresentamos um modelo desconstruído em que o Higgs é um pseudo bóson de Nambu Goldstone. Esse modelo fornece um mecanismo dinâmico para localizar o Higgs próximo do N-ésimo sítio da teoria e garante um potencial finito.

Estudos futuros do modelo de desconstrução são necessários. Do ponto de vista da construção de modelos, ainda precisamos considerar a propagação dos férmions no quiver para gerar um potencial para o Higgs capaz de realizar corretamente a quebra da simetria eletrofraca, assim como estudar como o potencial varia com o número de sítios. Esses resultados serão publicados em um trabalho atualmente em fase de preparação [32]. Outras questões possíveis a estudar são como obter as massas e matriz de mistura corretas para o setor de léptons, e também estudar a fenomenologia dos modos massivos.

Considerando estudos mais teóricos, sabemos que modelos em $A d S_{5}$ são na verdade uma descrição holográfica de teorias quase conformes fortemente acopladas, pela correspondência $A d S / C F T$ [41]. Seria muito interessante estudar se os modelos de descontrução de poucos sítios aqui apresentados também podem ser pensados como uma ferramenta que descreve algum tipo de sistema fortemente acoplado.

Futuramente esperamos prosseguir nossos estudos na construção de modelos e fenomenologia para a física na escala TeV. Com o LHC, temos a oportunidade de explorar nova física e desvendar a origem da quebra de simetria eletrofraca, o que torna essa uma época muito importante para a física de partículas. 


\section{Bibliografia}

[1] M. E Peskin e D. V. Schroeder. An Introduction To Quantum Field Theory. Westview Press, 1995.

[2] J. L. Hewett. The Standard Model and why we believe it. SPIRES Conference, C97/06/01, 1997.

[3] LEP Electroweak Working Group (LEP EWWG). Disponível em http://lepewwg.web.cern.ch/LEPEWWG/.

[4] Tevatron Electroweak Working Group (TEV-EWWG). Disponível em http:/ / tevewwg.fnal.gov/.

[5] K. Nakamura et al. (Particle Data Group). Review of particle physics. Journal of Physics G: Nuclear and Particle Physics, 37(075021), 2010. Disponível em http:/ / www-pdg.lbl.gov/.

[6] The ATLAS Collaboration. Observation of a new particle in the search for the Standard Model Higgs boson with the ATLAS detector at the LHC. Physics Letters B, 716:1-29, 2012.

[7] The CMS Collaboration. Observation of a new boson at a mass of $125 \mathrm{GeV}$ with the CMS experiment at the LHC. Physics Letters B, 716:30-61, 2012. 
[8] John F. Donoghue. General relativity as an effective field theory: The leading quantum corrections. Phys.Rev., D50:3874-3888, 1994.

[9] E. Komatsu et al. (WMAP Collaboration). Five-year wilkinson microwave anisotropy probe (WMAP) observations: Cosmological interpretation. Astrophysical Journal Supplement Series, 180:330-376, 2009.

[10] S. Weinberg. The cosmological constant problem. Reviews of Modern Physics, 61:1-23, 1989.

[11] S. Weinberg. The cosmological constant problems. Palestra apresentada na Conferência Dark Matter, 2000. Disponível em http://arxiv.org/abs/astro$\mathrm{ph} / 0005265$.

[12] B. W. Lee, C. Quigg, e H. B. Thacker. Weak interactions at very high energies: The role of the Higgs-boson mass. Physical Review D, 16:1519-1531, 1977.

[13] J. D. Wells. Lectures on Higgs boson physics in the standard model and beyond. British Universities Summer School in Theoretical Elementary Particle Physics, 2009. Disponível em http://arxiv.org/abs/0909.4541v1.

[14] Gerard 't Hooft. Naturalness, chiral symmetry, and spontaneous chiral symmetry breaking. NATO Adv.Study Inst.Ser.B Phys., 59:135, 1980.

[15] Riccardo Barbieri e Alessandro Strumia. The 'LEP paradox'. 2000. e-Print: hep-ph/0007265.

[16] The CDF Collaboration. Evidence for a mass dependent forward-backward asymmetry in top quark pair production. Physical Review D, 83(112003), 2011. 
[17] The D0 Collaboration. Forward-backward asymmetry in top quark-antiquark production. 2011. Disponível em http://lss.fnal.gov/archive/test-preprint/fermilab-pub-11-347-e.shtml.

[18] J. M. Campbell e R. K. Ellis. Radiative corrections to Z b anti-b production. Physical Review D, 62(114012), 2000.

[19] O. Antuñano, J. H. Kühn, e G. Rodrigo. Top quarks, axigluons and charge asymmetries at hadron colliders. Physical Review D, 77(014003), 2008.

[20] CMS collaboration. Measurement of the charge asymmetry in top quark pair production with the CMS experiment. Disponível em http://inspirehep.net/record/925254/files/TOP-10-010-pas.pdf, 2011.

[21] The ATLAS collaboration. Measurement of the charge asymmetry in top quark pair production in pp collisions at $\sqrt{s}=7 \mathrm{TeV}$ using the ATLAS detector. ATLAS-CONF-2011-106, 2011. Disponível em http://cdsweb.cern.ch/record/1372916.

[22] G. Burdman, L. Lima, e R. D. Matheus. New strongly coupled sector at the Tevatron and the LHC. Physical Review D, 83(035012), 2011.

[23] Marcela Carena, Ian Low, e Carlos E.M. Wagner. Implications of a Modified Higgs to Diphoton Decay Width. JHEP, 1208:060, 2012.

[24] Nima Arkani-Hamed, Kfir Blum, Raffaele Tito D'Agnolo, e Jiji Fan. 2:1 for Naturalness at the LHC? 2012. e-Print: hep-ph/1207.4482.

[25] Dean Carmi, Adam Falkowski, Eric Kuflik, Tomer Volansky, e Jure Zupan. Higgs After the Discovery: A Status Report. JHEP, 1210:196, 2012. 
[26] Alexandre Alves, A.G. Dias, E. Ramirez Barreto, C.A. de S. Pires, Farinaldo S. Queiroz, et al. Explaining the Higgs Decays at the LHC with an Extended Electroweak Model. Eur.Phys.J., C73:2288, 2013.

[27] G. A. Burdman, Z. Chacko, R. Harnik, e L. Lima. Em preparação.

[28] L. Randall e R. Sundrum. Large mass hierarchy from a small extra dimension. Physical Review Letters, 83:3370-3373, 1999.

[29] N. Arkani-Hamed, A. G. Cohen, e H. Georgi. (De)constructing dimensions. Physical Review Letters, 86:4757-4761, 2001.

[30] C. T. Hill, S. Pokorski, e J. Wang. Gauge invariant effective Lagrangian for Kaluza-Klein modes. Physical Review D, 64(105005), 2001.

[31] G. A. Burdman, L. Lima, e N. F. de Sá. Full-Hierarchy Quiver Theories of Electroweak Symmetry and Fermion Masses. Journal of High Energy Physics, $2013,2013$.

[32] G. A. Burdman, L. Lima, e N. F. de Sá. The Higgs as a Light Composite in Full-Hierarchy Quiver Theories. Em preparação.

[33] N. Arkani-Hamed, S. Dimopoulos, e G. Dvali. The hierarchy problem and new dimensions at a millimeter. Physics Letters B, 429:263-272, 1998.

[34] L. Randall e R. Sundrum. An alternative to compactification. Physical Review Letters, 83:4690-4693, 1999.

[35] T. Gherghetta e A. Pomarol. Bulk fields and supersymmetry in a slice of AdS. Nuclear Physics B, 586:141-162, 2000. 
[36] A. Pérez-Lorenzana. An introduction to extra dimensions. Journal of Physics: Conference Series, 18, 2005.

[37] T. Gherghetta. Les Houches lectures on warped models and holography. Les Houches 2005, Particle physics beyond the Standard Model, pages 263-311, 2005.

[38] T. Gherghetta. TASI lectures on a holographic view of beyond the standard model physics. Theoretical Advanced Study Institute 2010, 2010. Disponível em http://arxiv.org/abs/hep-ph/1008.2570.

[39] Y. Grossman e M. Neubert. Neutrino masses and mixings in non-factorizable geometry. Physics Letters B, 474:361-371, 2000.

[40] C. Csáki. TASI lectures on extra dimensions and branes. Theoretical Advanced Study Institute 2002, 2002. Disponível em http:/ /arxiv.org/abs/hep$\mathrm{ph} / 0404096$.

[41] J. M. Maldacena. The large N limit of superconformal field theories and supergravity. Advances in Theoretical and Mathematical Physics, 2:231-252, 1998.

[42] C. T. Hill e E. H. Simmons. Strong dynamics and electroweak symmetry breaking. Physics Reports, 381(4-6):235-402, 2003.

[43] Yutaka Hosotani e Mitsuru Mabe. Higgs boson mass and electroweakgravity hierarchy from dynamical gauge-Higgs unification in the warped spacetime. Phys.Lett., B615:257-265, 2005.

[44] Naoyuki Haba, Shigeki Matsumoto, Nobuchika Okada, e Toshifumi Yamashita. Effective Potential of Higgs Field in Warped Gauge-Higgs Unification. Prog.Theor.Phys., 120:77-98, 2008. 
[45] Lisa Randall, Yael Shadmi, e Neal Weiner. Deconstruction and gauge theories in AdS(5). JHEP, 0301:055, 2003.

[46] Gustavo Burdman, Leandro Da Rold, Oscar Eboli, e Ricardo D’Elia Matheus. Strongly Coupled Fourth Generation at the LHC. Phys.Rev., D79:075026, 2009.

[47] Antonio Delgado e Adam Falkowski. Electroweak observables in a general 5D background. JHEP, 0705:097, 2007.

[48] S. Casagrande, F. Goertz, U. Haisch, M. Neubert, e T. Pfoh. Flavor Physics in the Randall-Sundrum Model: I. Theoretical Setup and Electroweak Precision Tests. JHEP, 0810:094, 2008.

[49] S. L. Glashow, J. Iliopoulos, e L. Maiani. Weak interactions with leptonhadron symmetry. Physical Review D, 02, 1970.

[50] G. A. Burdman. Constraints on the bulk standard model in the RandallSundrum scenario. Physical Review D, 66(7), 2002.

[51] G. A. Burdman. Flavor violation in warped extra dimensions and CP asymmetries in B decays. Physics Letters B, 590:86-94, 2004.

[52] S. J. Huber. Flavor violation and warped geometry. Nuclear Physics B, 666:269-288, 2003.

[53] K. Agashe, G. Perez, e A. Soni. Flavor structure of warped extra dimension models. Physical Review D, 71(016002), 2005. 
[54] A. Delgado, A. Pomarol, e M. Quiros. Electroweak and flavor physics in extensions of the standard model with large extra dimensions. Journal of High Energy Physics, 01, 2000.

[55] C. Csáki, A. Falkowski, e A. Weiler. The flavor of the composite pseudogoldstone higgs. Journal of High Energy Physics, 09(08), 2008.

[56] J. Santiago. Minimal flavor protection: a new flavor paradigm in warped models. Journal of High Energy Physics, 12(046), 2008.

[57] C. Csáki, A. Falkowski, e A. Weiler. Simple flavor protection for the RandallSundrum model. Physical Review D, 80(016001), 2009.

[58] Y. Bai, G. Burdman, e C. T. Hill. Topological Interactions in Warped Extra Dimensions. Journal of High Energy Physics, 2(49), 2010.

[59] N. F. de Sá. Desconstrução dimensional e violação de sabor. Dissertação de mestrado, Universidade de São Paulo, 2011.

[60] J. F. Donoghue, E. Golowich, e B. R. Holstein. Dynamics of the Standard Model. Cambridge University Press, 1994.

[61] Christopher D. Carone, Joshua Erlich, e Brian Glover. Dynamically warped theory space and collective supersymmetry breaking. JHEP, 0510:042, 2005.

[62] Adam Falkowski e Hyung Do Kim. Running of gauge couplings in AdS(5) via deconstruction. JHEP, 0208:052, 2002.

[63] Andrey Katz e Yael Shadmi. Gauge theories in AdS(5) and fine-lattice deconstruction. JHEP, 0411:060, 2004. 
[64] W. Hahn. Die mechanische Deutung einer geometrischen Differenzgleichung. Zeitschrift für Angewandte Mathematik und Mechanik, 33:270-272, 1953.

[65] H. Exton. q-hypergeometric functions and applications. Ellis Hood, 1983.

[66] J. de Blas, A. Falkowski, M. Pérez-Victoria, e S. Pokorski. Tools for deconstructing gauge theories in $A d S_{5}$. Journal of High Energy Physics, 8(61), 2006.

[67] R. F. Swarttouw. The Hahn-Exton q-Bessel Function. Doutorado, Universidade de Tecnologia Delft, 1992. Disponível em http:/ /www.narcis.nl/publication/RecordID/oai\%3Atudelft.nl\%3Auuid\% 3A0bfd7d96-bb29-4846-8023-6242ce15e18f.

[68] Yonatan Kahn e Jesse Thaler. Locality in Theory Space. JHEP, 1207:007, 2012.

[69] H. J. Rothe. Lattice Gauge Theories. World Scientific, 2005.

[70] David B. Kaplan. Chiral Symmetry and Lattice Fermions. pages 223-272, 2009. e-Print: hep-lat/0912.2560.

[71] Martin Luscher. Chiral gauge theories revisited. pages 41-89, 2000. e-Print: hep-th/0102028.

[72] M. Bona et al. [UTfit Collaboration]. Model-independent constraints on $\Delta$ $\mathrm{F}=2$ operators and the scale of new physics. Journal of High Energy Physics, 049, 2008.

[73] V Bertone, N. Carrasco, M. Ciuchini, P. Dimopoulos, R. Frezzotti, V. Gimenez, V. Lubicz, e G. Martinelli et al. Kaon Mixing Beyond the SM from Nf=2 tmQCD and model independent constraints from the UTA. arXiv:1207.1287 [hep-lat]. 
[74] Gerhard Buchalla, Andrzej J. Buras, e Markus E. Lautenbacher. Weak decays beyond leading logarithms. Rev.Mod.Phys., 68:1125-1144, 1996.

[75] Michael E. Peskin e Tatsu Takeuchi. Estimation of oblique electroweak corrections. Phys.Rev., D46:381-409.

[76] M. Baak, M. Goebel, J. Haller, A. Hoecker, D. Kennedy, R. Kogler, K. Moenig, e M. Schott et al. The Gfitter Collaboration. The Electroweak Fit of the Standard Model after the Discovery of a New Boson at the LHC. arXiv:1209.2716 [hep-ph].

[77] Kaustubh Agashe, Antonio Delgado, Michael J. May, e Raman Sundrum. RS1, custodial isospin and precision tests. JHEP, 0308:050, 2003.

[78] F. Bazzocchi e M. Fabbrichesi. The little hierarchy problem for new physics just beyond the LHC. 2012. e-Print: hep-ph/1212.5065.

[79] Hsin-Chia Cheng e Ian Low. TeV symmetry and the little hierarchy problem. JHEP, 0309:051, 2003.

[80] Tony Gherghetta, Benedict von Harling, e Nicholas Setzer. A natural little hierarchy for RS from accidental SUSY. JHEP, 1107:011, 2011.

[81] Bohdan Grzadkowski e Jose Wudka. Naive solution of the little hierarchy problem and its physical consequences. Acta Phys.Polon., B40:3007-3014, 2009.

[82] Gustavo Burdman e Yasunori Nomura. Unification of Higgs and gauge fields in five-dimensions. Nucl.Phys., B656:3-22, 2003. 
[83] Lawrence J. Hall, Yasunori Nomura, e David Tucker-Smith. Gauge Higgs unification in higher dimensions. Nucl.Phys., B639:307-330, 2002.

[84] Ignatios Antoniadis, K. Benakli, e M. Quiros. Finite Higgs mass without supersymmetry. New J.Phys., 3:20, 2001.

[85] G.R. Dvali, S. Randjbar-Daemi, e R. Tabbash. The Origin of spontaneous symmetry breaking in theories with large extra dimensions. Phys.Rev., D65:064021, 2002.

[86] Adam Falkowski. About the holographic pseudo-Goldstone boson. Phys.Rev., D75:025017, 2007.

[87] N. Arkani-Hamed, A. G. Cohen, e H. Georgi. Electroweak symmetry breaking from dimensional deconstruction. Physics Letters B, (513):232-240, 2001.

[88] Sidney R. Coleman e Erick J. Weinberg. Radiative Corrections as the Origin of Spontaneous Symmetry Breaking. Phys.Rev., D7:1888-1910, 1973.

[89] Martin Schmaltz e David Tucker-Smith. Little Higgs review. Ann.Rev.Nucl.Part.Sci., 55:229-270, 2005.

[90] Bohdan Grzadkowski e Jose Wudka. 5-Dimensional Difficulties of GaugeHiggs Unifications. Phys.Rev.Lett., 97:211602, 2006.

[91] R. Jackiw. Functional evaluation of the effective potential. Phys.Rev., D9:1686, 1974.

[92] S. Pokorski. Gauge Field Theories. Cambridge Monographs On Mathematical Physics, 1987. 
[93] Antonio Dobado, Lourdes Tabares-Cheluci, e Siannah Penaranda. The Higgs effective potential in the Littlest Higgs model at the one-loop level. Phys.Rev., D75:083527, 2007.

[94] A. Dobado, A. Gómez-Nicola, A.L. Maroto, e J.R. Pelaez. Effective Lagrangians for the Standard Model. Springer-Verlag, 2007.

[95] S. Coleman. Aspects of Symmetry. Cambridge University Press, 1988.

[96] Andrey Katz e Yael Shadmi. Gauge theories in AdS(5) and fine-lattice deconstruction. JHEP, 0411:060, 2004. 